\title{
An Experimental Study of Neutron Noise with Criticality Safety Applications in Mind
}

\author{
Charles S. Barnett
}

(Ph.D. thesis)

\section{Manuscript date: November 1985}

\section{DISCLAIMER}

This report was prepared as an sccount of work sponsored by an agency of the United States Government. Neither the United States Government nor any agency thereof, nor any of their employes, makes any warranty, express or impiied, or assumes any legal liability or responsibility for the accuracy, completeness, or usefulness of any information, apparatus, product, or process disclosed, or represents that its use would not infringe privately owned rights. Reference herein to any specific commercial product, process, or service by trade name, trademark, manufacturer, or otherwise does not necesserily constitute or imply its endorsement, recommendation, or favoring by the United States Government or any agency thereof. The views and opinions of authors expressed herein do not necessarity state or reflect those of the United States Government or any agency thereof.

\section{LAWRENCE LIVERMORE NATIONAL LABORATORY University of California - Livermore, California - 94550}




\section{An Experimental Study of Neutron Noise with Criticality Safety Applications in Mind}

By

\section{CHARLES SIDNEY BARNETT}

B.S. (University of California, Berkeley) 1953

M.S. (University of California, Berkeley) 1956

M.S. (California State University, Hayward) 1970

\section{DISSERTATION}

Submitted in partial satisfaction of the requirements for the degree of

DOCTOR OF PHILOSOPHY

in

Engineering-Applied Science

in the

GRADUATE DIVISION

of the

UNIVERSITY OF CALIFORNIA

DAVIS

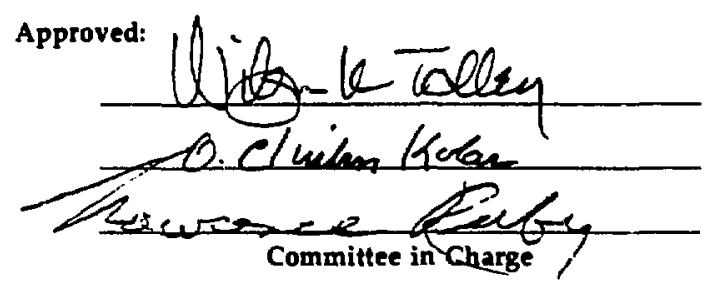

Deposited in the Universily Library 


\title{
An Experimental Study of Neutron Noise with Criticality Safety Applications in Mind
}

\begin{abstract}
I studied the statistics of detected neutrons that leaked from four subcritical reflected, enriched-uranium assemblies, to explore the feasibility of developing a criticality warning system based on neutron noise analysis. The calculated multiplication factors of the assemblies are $0.59,0.74,0.82$, and 0.92 . I studied three possible discriminators, i.e., three signatures that might be used to discriminate among assemblies of various multiplications. They are (1) variance-to-mean ratio of the counts in a time bin (V/M), (2) covariance-to-mean ratio of the counts in a common time bin from two different detectors $(\mathrm{C} / \mathrm{M}),(3)$ covariance-to-mean ratio of the counts from a single detector in two adjacent time bins of equal length, which I call the serial-covariance-to-mean ratio (SC/M). The performances of the three discriminators were not greatly different, but a hierarchy did emerge: $S C / M \geq V / M \geq C / M$. An example of some results: in the neighborhood of $k=0.6$ the $\Delta k$ required for satisfactory discrimination varies from about $3 \%$ to $7 \%$ as detector solid angle varies from $19 \%$ to $5 \%$. In the neighborhood of $k=0.8$ the corresponding $\Delta k s$ are $1 \%$ and $2 \%$. The noise analysis techniques studied performed well enough in deeply subcritical situations to deserve testing in an applications environment. They have a good chance of detecting changes in reactivity that are potentially dangerous. One can expect sharpest results when doing comparisons, i.e., when comparing two records, one taken in the past under circumstances known to be normal and one taken now to search for change.
\end{abstract}




\section{Introduction}

Current practitioners of the nuclear criticality safety art employ two basic tools at the work site: (1) administrative and mechanical controls of magnitudes, positions, and movements of masses of fissile materials, and (2) criticality alarm systems. If the first tool works, there is zero nuclearcriticality consequence. If the second is activated, a catastrophic situation has developed. Note the wide gap between these two circumstances. Can we bridge this gap? Can we develop instruments and methods to warn operators who work with fissile materials of undesired but less than catastrophic increases in muitiplication?

Suppose a flexible system of instruments and methods were developed to sense changes in reactivity. For convenient reference, give the system a name: criticality warning system (CWS). Pussible safety applications of a CWS are (1) monitoring reactivity changes at various points in a chemical processing line at which an excess mass of fissile materials might accumulate and lead to near-critical configurations, (2) monitoring reactivity changes at similar points in a fuel fabrication facility, (3) helping to minimize the probability of inducing a criticality during recovery from accident situations, such as fire, flood, earthquake, criticality.

Tied to the safety implication of a CWS is an economic one. The reactivities of process configi:rations are of ten difficult to calculate reliably; hence, design and operational safety limits are set at quite conservative levels, levels that constrain production rates. Perhaps a CWS would safely allow relaxation of limits imiposed for criticality safety reasons.

I undertook the work reported here to explore the feasibility of developing a criticality warning system, of closing the gap referred to above. I hasten to add that the gap did not close, but I think it is perhaps a fraction smaller.

\section{A Game}

To set the scene, consicier the following imaginary situation. In a laboratory I have set up a fixcd-geometry neutron counting arrangement. The counting apparatus records the time at which a detection occurs and the identity of the detector if more than one is used. In the stockroom you have available $n$ subcritical assemblies of fissile material. Their multiplications are $M_{1}>M_{2}>\ldots>M_{11}$, and each assembly contains a source of neutrons. All are packaged in identical containers. You have adjusted the neutron source strengths in each assembly so that all assemblies give a cominon mean count rate when placed in our fixed-geometry counting apparatus.

We play a game as follows: you use some kind of device, a roulette wheel perhaps, to randonly choo" al asiembly from the stockroom and place it into the counting apparatus. I turn on the counter until $K$ counts, say, are recorded. You spin your roulette wheel again and select another (or the same) assembly and place it into the counting apparatus. Again, I record $K$ counts.

My problem is to try to decide, from the two records i have, whether or not you placed different assemblies into the apparatus. Observe that the problem is constructed so that I cannot use count rate as a discriminator. If any useful information is available, it is stored in the way in which the detections are spread along the time line. And it is known that useful information is stored in such records. The statistical properties of a high-multiplication record are different from those of a low-multiplication record.

One way l might proceed is to select a statistical parameter that tends to be high in highmultiplication records and low in low-multiplication records, and design a processor to estimate that parameter. I then apply the processor to the two records and announce "different assemblies" if the two estimates are sufficiently far apart and "same assembly" otherwise. The more I know about your random selection scheme and about the $M_{i}$, the better I am able to determine what "sufficiently far apart" should be. Also, "sufficiently far apart" will depend upon the reward and penalty agreements. But, because of the inherent statistical nature of the source emission and fission frocesses, my decision procedure can never be perfect. 
The game just described is an idealization of the problem of detecting changes in reactivity at fissile material work stations or processing points. The roulette wheel and the set of subcritical asse - jlies of various multiplications simulate the uncertainties that always accompany machine and human interaction. The requirement that count rate cannot be used as a discriminator corresponds to the fact that at the workplace neutron source strengths will vary in ways not controllable by the observer. For example, increased count rate does not necessarily imply increased reactivity; it may just mean that a stronger source of neutrons has entered the area of interest or that the extraneous background rate has increased because of additional neutron emitters brought into the vicinity of the workplace.

\section{The Work Done}

In the briefest of terms, here is what I did. I assembled enriched uranium subcritical units of different multiplications, drove them with a neutron source, and recorded detection times of leaked neutrons. I used four assemblies, the most reactive having a multiplication of about 10 , and two neutron sources, one nonfission and one fission. Sixteen detectors were placed near the assembly, and eaci time a detection was recorded, the identity of the firing detector was also recorded.

All these records were then stored on a medium accessible to a CDC 7600 computer. I then subjected the records to various algorithms designed to extract statistical signatures that could be tested for their ability to discriminate among various levels of multiplication. In accord with my comments above, count rate was not used as a discriminator in any of the algorithms.

I concentrated effort on three discriminators that l found to be serviceable. They are (1) variance-to-mean ratio of the counts in a time bin, V/M, (2) covariance-to-mean ratio of the counts in a common time bin from two different detectors, $C / M$, (3) covariance-to-mean ratio of the counts from a single detector in two adjacent time bins of equal length (which I call the serialcovariance-to-mean ratio), SC/M. Notice that with software l could arrange for any subset of the 16 detectors to become a single detector.

The fuel for the subcritical assembly consists of eight enriched $(93.2 \%)$ uranium shells-four sets of twins. Inside diameter of the smallest pair is $11.8 \mathrm{~cm}$; outside diameter of the largest pair is $15.9 \mathrm{~m}$. The shells nest snugly, making it convenient to assemble, starting from smallest to largest, a sequence of one-shell, two-shell, three-shell, and four-shell configurations. That sequence of assemblies was used for the work reported here. The corresponding sequence of uranium masses was 5.49, 11.67, 16.31, and $22.64 \mathrm{~kg}$. Each assembly was reflected internally by an $11.8-\mathrm{cm}$. u.d., $1.9-\mathrm{cm}$-thick spherical shell of acrylic resin and reflected externallv by a $16.0-\mathrm{cm}-\mathrm{i} . \mathrm{d} ., 10-\mathrm{cm}-$ thick spherical shell of acrylic resin. The centrai void of $8-\mathrm{cm}$ diameter accommodated the driving neutron sources. The calculated multiplication factors for the four assemblies were $0.59,0.74,0.82$, and 0.92. Calculated median energies of neutrons inducing fission varied from $0.4 \mathrm{keV}$ for the onefuel-shell assembly to $100 \mathrm{keV}$ for the four-fuel-shell assembly.

Leaking neutrons were detected by two identical planar arrays of helium-3 detectors, eight detectors per bank. Detectors were 2 in. in diameter by 36 in. long; they were backed by a 2-in.thick block of polyethylene. The solid angle subtended at the center of the assembly by a single detector averaged $1.2 \%$.

The detertion time and detector number data pairs were recorded on floppy disks. For any given assembly configuration, a single run terminated when one floppy disk was full of data. Typically, about 120,000 counts were recorded per run. The number of counts recorded varied slightly from run to run because some disk space was required to record clock cycle completion times. Hence, disks from low-count-rate runs contained slightly fewer counts than disks from highcount-rate runs. The sum of the count rates from all 16 detectors varied in the neighborhood of a few kilohertz when assemblies were driven by either of two neutron sources, fission or nonfission, each emitting about $10^{4}$ neutrons per second. For each major configuration of interest, I made 20 runs, giving 20 disks of data. 
Now I can say a bit more about how discriminator comparisons were made. Focus attention on one discriminator, V/M, say. The following steps gave me one V/M estimate. Pick an assembly configuration, pick one data disk for that configuration, and decide on a detector subset (all 16 , for example). Calculate variance-to-mean ratios for several different time bin widths, for example, 250 $\mu \mathrm{s}, 500 \mu \mathrm{s}$.... Perform a two-parameter, least-squares fit of these data to the theoretical curve that is derived later in this report. One of the parameters is our V/M estimator; the other is the assembly decay time constant. Repeat the process for the remaining 19 disks of data taken under identical circumstances. I now have 20 samples of the V/M estimator, generally all different because of the randomness inherent in the processes from which the data were extracted. Fit a cumulative probability distribution function to these 20 samples.

Repeat the process sketched in the previous paragraph for the other three assembly configurations. We now have cumulative distribution functions for the four assemblies with fucl masses of $5.49,11.67,16.31$, and $22.64 \mathrm{~kg}$ of uranium. Interpolate between these four mass points and get a recipe that assigns a V/M cumulative distribution for any fuel mass between $5.49 \mathrm{~kg}$ and $22.64 \mathrm{~kg}$ (hence a recipe for 7 ny calculated multiplication factor between 0.59 and 0.92 ).

I now have sufficient information to predict the performance of a V/M threshold decision procedure. I illustrate with an example. Suppose I wish to find out how well I can discriminate between a $6.0-\mathrm{kg}$-mass assembly and a $6.5-\mathrm{kg}$-mass assembly with my apparatus placed as it was when all the data were collected. I generate the V/M distribution functions for the $6.0-\mathrm{kg}$ and the $6.5-\mathrm{kg}$ cases. Then, by moving the V/M threshold from low to high values, I generate a curve of true high decision probability vs false high decision probability. That is, I can compute, for any threshoid setting, two important conditional probabilities: (1) the probability that a disk of data from a $6.5 \mathrm{~kg}$ assembly, when processed as described above, will yield a V/M value greater than that threshold (true high decision probability), and (2) the probability that a disk of data from a 6.0$\mathrm{kg}$ assembly, when so processed, will yield a V/M value greater than that threshold (false high decision probability). A set of such pairs generates a curve inside the unit square, which curve I call the decision rule performance characteristic (DRPC). From that curve I can determine the false high decision probability that I must accept for any desired true high decision probability.

l constructed DRPCs as just described for the V/M discriminator for three sets of detectors (all 16 , a subset of 8 , and a subset of 4), for fuel mass pairs in the neighborhood of $6.0 \mathrm{~kg}$ and $16.0 \mathrm{~kg}$, and for two neutron sources (fission and nonfission). By similar means I generated corresponding DRPCs for the $\mathrm{C} / \mathrm{M}$ and $\mathrm{SC} / \mathrm{M}$ discriminators. Since the $\mathrm{C} / \mathrm{M}$ and $\mathrm{SC} / \mathrm{M}$ discriminators use covariance techniques, two detector subsets are required. The sets chosen were symmetric halves of the sets used for the V/M case. Also, each of the $\mathrm{C} / \mathrm{M}$ and SC/M techniques requires least-squares tits to unique theoretical functions that have time bin width for domain and discriminator value for range. Those functions are clerived in the main body of the text.

The DRPCs were then used as measures of goodness to arrive at the relative per.ormances of the three discriminators. They were also used to determine the minimum difference in mass ( $\Delta m$ ) and the corresponding minimum difference in the multiplication factor $(\Delta k)$ that must exist for satisfactory discrimination between two asser blies. By "satisfactory discrimination," I mean that the true high decision probability is to be essentially one and the false high decision probability is to be essentially zero.

The discriminators whose properties I investigated exploit the stochastic nature of the fission process and the stochastic nature of neutron emission from nonfission and fission neutron sources. Hence an interpretive probabilistic model is required. For the fast spectrum systems I studied, the no-delayed-neutrons, point model seemed to be adequate.

In the main body of the text I give a fairly careful derivation of the various theoretical relations that I need. In the model I allow for two neutron detectors, a mixed neutron source (nonfission plus fission), and extraneous background flux on the detectors.

Here are the steps used to derive the needed relations: (1) write probability balance relations for the three-dimensional state space (one dimension for the neutron population and one dimension each for the two detectors), (2) pass to the limit of small time increment and obtain a triply infinite set of ordinary differential equations for the probability mass function that assigns probability to elements of the state space, (3) collapse that array of ordinary differential equations to one 
partial differential equation for the probability generating function, (4) extract ficun that partial differential equation a system of ordinary differential equations for selected low-order moments, (5) solve the system derived in step 4. Finally, selected members of that solution set are combined to yield the theoretical expressions for the three discriminators: variance-to-mean ratio, covariance-to-mean ratio, and serial-covariance-to-mean ratio. 


\section{Background}

The subject of neutron noise analysis began at the Los Alamos Scientific Laboratory during World War II. A list of names of the physicists who made the original contributions (E. Fermi, R. Feynman, F. de Hoffman, B. Rossi, and R. Serber) includes some that are famous. Apparently, de Hoffmann was the first to discuss those contributions in the open literature.' The contents of his report were extracted from two internal reports. ${ }^{21}$ He discusses theoretical aspects of the neutronics of the Los Alamos Water Boiler (LOPO) reactor, and, among other things, he derives an expression for the variance-to-mean ratio of the counts in a time bin recorded by a detector expused to leaking neutrons. The other description of the Los Alamos wartime contributions that appeared in the open literature is by Feynman, de Hoffmann, and Serber. ' In that paper the authors describe the results of experiments that exploited neutron-intensity fluctuations to measure the dispersion in the number of neutrons emitted in a uranium-235 fission. Variance-to-mean ratio of the counts in a time bin vs time-bin width is the statistical function that was used to estimate the dispersion. The authurs recorded individual neutron counts from a $B F_{3}$ counter situated to detect leaked neutrons. The detection times; were recorded on a film strip that moved across the face of a cathode ray tube.

The Rossi-cx technicue for measuring die-away times of multiplying assemblies was also conceived and applied at Los Alamos. The name of the technique comes from a suggestion by Bruno Rossi that the ex (reciprocal of the die-away time) of a multiplying assembly cuuld be inferred by constructing the auto-correlation function of a sequence of neutron detections. The first Rossi- $\alpha$ measurements reported in the open literature were done on the Godiva assembly at Los Alamos by J.D. Orndoff.

The wartime work at Los Alamos was the beginning of what is now a mature endeavor called "reactor noise analysis" or "neutron noise analysis " The scope of the field can be appreciated by the number of special topical meetings that have been held, the number of major review articles that have been published, and the number of books that have been published. A recent review article by N. Pacilio et al." cites the following list: (1) conferences-Florida 1963, " Florida 1966, "Petten 1967," Tokyo/Kyoto 1968, "' Rome 1974"; (2) reviews-Seifritz and Stegemann 1971, " Saito 1974"; (3) books-Thie 1963, "Pacilio 1969, " Uhrig 1970, "Williams 1974. " A recent addition to the list of books is the une by Thie published in 1981."

For some time after the Los Alamos work became public, most work in the field of neutron moise was restricted to the zero-power case, the case in which the only sources of luctuations in neutron population are those inherent in the driving neutron source, the fission process, the neuiron-transport process, and the neutron-capture process. The Rossi- $\alpha$ method and the Fevnman iariance-to-mean method were used to estimate assembly characteristics. and various workers exploited other statistical features of the neutron detection process. Those additional methods included but were not limited to (1) measurement of second moments of the difference in counts in consecutive equal-time intervals, " $(2)$ zero probability method, in which one measures the fraction of empty time channels vs channel width," (3) interval distribution method, in which one constructs an empirical distribution of lengths of time intervals between counts. ${ }^{\prime \prime 2}=$ This reference to methods other than the Rossi- $\alpha$ and the Feynman variance-to-mean is representative, not exhaustive.

As the nuclear industry began to mature, the interest of noise analysts shifted to power reactor applications where additional noise sources existed: boiling instability, core barrel motion, tuel motion, individual fuel-rod motion, control-rod vibration. Reference 18 reviews power reactor noise applications. I say nothing further about such applications since they are not relevant to the work reported here.

In recent years interest in zero-power neutron noise analysis has been somewhat rekindled but for reasons and with emphases different from the original ones. The new interest arises because of international nuclear safeguards concerns that require nuclear material assay techniques. Proceedings of three international meetings on safeguards technology afford a view of work on assay and accountability methods. ${ }^{24.5 .5}$ Two papers in particular from those proceedings, authored by Los Alamos workers, display theoretical approaches that reflect the early neutron noise 
work at Los Alamos. ${ }^{37.24}$ For safeguards work based on neutron detection, neutron multiplication in the sample is a nuisance, since it interferes with the usual assay techniques.

The work on which I report here is concerned with away-from-reactor, highly subcritical situations. Despite the general concentration of interest in the power reactor area, a steady stream of papers on neutron noise analysis of subcritical assemblies has emerged since the early seventies frum Mihalczo and associates at Oak Ridge National Laboratory. ${ }^{*-10}$ Mihalczo drives assemblies with a californium-252 source embedded in a detector that records the time of a spontaneous tissiun. That time, the start time of a random number of neutron chains, triggers electronics that periorm frequency domain analysis. The method requires measurement of the cross-power spectral density between a pair of detectors located inside or near the fissile assembly and the crosspower spectral density between each of those detectors and the detector within which the californium-252 source is deposited. Mihalczo claims that for some subcritical assemblies his method can be used to check calculations of subcritical reactivities."

More akin to the present work in method and somewhat akin in goal is the work of Ruby and students at the University of California at Berkeley. Their research is reported in a seyuence of papers that span the period from 1967 to 1977.11.th These papers discuss time-domain technicjues (primarily variance-to-mean ratio) for inferring the reactivity of slightly subcritical reactors. Inclusion of the effects ot delayed neutrons was important in their work because they were interested in measuring the reactivity of slightly subcritical systems with die-away times comparable to delayed neutron mean lifetimes. Most of the papers in the sequence by Ruby et al. are theoretical in tone, but two display some experimental results obtained with the AGN201 and TRIGA MARK III reactors." "Reasonable agreement with control-rod calibration data was obtained from their noise analysis results of experiments on the TRIGA reactor.

The general approach, point of view, language, and notation that I employ in constructing the probabilistic model are common in present-day literature on the theory of stochastic processes. See, for example, the two books by P. G. Hoel, S. C. Port, and C. J. Stone. ${ }^{\text {tin }}$ The inspiration and some of the notation for my approach to the derivation of the probability balance relations comes from a book by J. Lewins. "The general method has been known to mathematicians for a long time. The probability balance relations I derived correspond to what probabilists call forward Kolmogorov relations." They were first applied to neutron noise analysis by Courant and Wallace. "In deriving the expression for the serial-covariance-to-mean ratio, I found the book by D. R. Cox and P. A. Lewis to be useful."

1 borrowed from communication and radar engineers the idea of using decision rule performance characteristics (DRPCs) for comparing discriminator performances. Engineers use what they call receiver operating characteristics (ROCs) as measures of pertormance for detection systems. ROCs are plots of detection probability vs false alarm probability; hence DRPCs are equivalent. $A$ good discussion of ROC.s and associated ideas appears in a book by Whalen."

The multiplying assembly 1 used for the experiments was built for criticality safety training purposes. It is used at cawrence Livermore Nitional Laboratory (LLNL) to give fissile material handlers an upportunity to demunstrate for the:nselves how variations in fuel mass, moderator mass, and reflector thickness affect multiplication.

The data-collection system is the second in a sequence of two neutron statistics analyzers that were designed and built at LLNL. The first instrument is described elsewhere. ${ }^{4}$ Except for changes that experience with the first instrument dictated, the second is similar to the first. Development of the first instrument was directed by A. Zolnay (LLNL); H. Spracklen (LLNL retired) was the design engineer. The helium-3 tube bank design is patterned after a Los Alamos design. Prior to development of the first instrument, E. J. Dowdy and memhers of Q-2 group at Los Ala. mos gave us helpful advice and counsel. 


\section{Probabilistic Model}

The deterministic version of the model I enploy is known by various names: point model, lumped model, one-speed lumped model, space-and-entrgy averaged model. In our idealization all chain carriers have equivalent properties-a neuiron exists or not. No account is taken of the fact that neutrons have space and velocity descriptors. One further assumption: no delayed neutrons.

The no-delayed-neutrons model is adequate for the work reported here, work done with fast, deeply subcritical - isemblies. The measured die-away times of the assemblies were in the neighborhood of 300 to $400 \mu \mathrm{s}$, and the maximum time-bin width used in the measurements was $5000 \mu \mathrm{s}$. These times are short compar $d$ to the mean life of the shortest uranium-235 delayed group ( $-0.3 \mathrm{~s})$. If one were working with systems with die-away times equal to or greater than delayed-group mean lives, then the model would have to be modified to include delayed neutron effects. In iriticality safety applications, such a circumstance might arise if one were dealing with moderated systems (chemical processing plant applications come to mind). Also, die-away times lunger than those experienced in the present work could arise with fast systems if very high multiplications were encountered (much higher than the maximum of -10 experienced in the present work). However, a multiplication of 10 , although low to a reactor uperator or a critical assembly laboratory experimenter, is high for the applications I have in mind.

\section{Preliminaries}

Before starting construction of the model, I dispense with some mathematical preliminaries and introduce some notalional conventions.

For the mathematical model we are about to cunstruct, several random variables will be introduced. Hence, for the remainder of the model development, assume the existence of an underlying probability space, $(\Omega, \therefore, P)$, on which all random variables are defined. In this probability triple

$\Omega=$ sample space (ensemble),

$\therefore=\mathrm{a} \sigma$-field of subsets of $\Omega$,

$P=a$ probability measure with domain $\therefore$ and range the closed unit interval, i.e., $P_{2}>\rightarrow$ $[0,1]$.

Then a generic random variable ( $X$, say) is a map from $\Omega$ to $S_{X}$, where $S_{X}$ (state space for $X$ ) is tome subset of the real numbers. Similarly, a generic ranctom point ( $X$, say) is a map from $\Omega$ to $S_{X}$, where $S_{X}$ is some subset of 11 -dimensional Euclidean space.

Those randum variables whose state space is the set of non-negative integers (most that will appear are in this class) will be described by a probability mass function (PMF) or a prubability generating function (PGF). The PMF that describes $X$ will usually be denoted by $\mu_{X}$, where

$p_{Y}(j)=P[X=j]=P\{\omega \in \Omega: X(\omega)=j\}, j \in\{0,1,2, \ldots$,$\} .$

In Eq. (1) the right-most entry is the full-blown measure-theoretic one and will be used only when and if thorough clarification is needed. The PGF that describes $X$ will usually be denoted by $g X$. where

$g x(s)=\left\langle s^{x}\right\rangle ; s \in[0,1]$

and $\langle\cdot\rangle$ represents the expectation operator. Exceptions to both Eqs. (1) and (2) will occur but will be explained at first appearance.

Similar notation will be used for describing functions of random points in spaces of dimension greater than one, but I will introduce it as needed. 


\section{Characterization}

Now, back to constructing the model. The system sketched in Fig. 1 is characterizable by cight fixed times and two random-variable describing functions:

$$
\begin{aligned}
\frac{n}{\tau_{C}} & =\begin{array}{l}
\text { mean rate of disappearance of neutrons from the assembly by parasitic capture } \\
\text { and nondetected leakage when the neutron population in the assembly is } n,
\end{array} \\
\frac{n}{\tau_{F}} & =\begin{array}{l}
\text { mean fission rate in the assembly fuel when the neutron population in the assem- } \\
\text { bly is } n,
\end{array} \\
\frac{n}{\tau_{\mathrm{L}}} & =\text { mean count rate in the L-counter caused by neutrons leaked from the assembly } \\
& \text { when the neutrull population in the assembly is } n,
\end{aligned}
$$

Our list of primitive, intrinsic descriptors is complete. One can invoke various neutronics models and express the $\tau^{\prime}$ 's in terms of cross sections, geometry of the assembly, and detector efficiency, but I priter to leave the characterization as it stands.
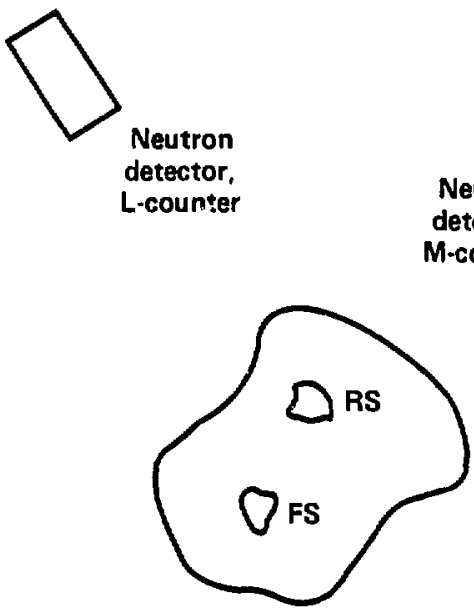

Multiplying assembly

Fig. 1. Schematic of the multiplying assembly and neutron detection system. RS represents the nonfission (random) source; FS represents the fission source. 
Until further notice, assume that the L-counter shown in Fig. 1 is turned off. The one-counter model lends itself to clear exposition and, when complete, can easily be extended to the twocounter case.

Now let

$N(t)=$ neutron population in the multiplying assembly at time $t . N(t)$ is a random variable.

$M(t)=$ number of counts accumulated in the M-counter by time $: M(t)$ is a random variable.

Observe that, for given $t, N(t)$ and $M(t)$ are indeed random variables, but $N$ and $M$ are random functions or stochastic processes.

Segments of realizations (sample paths) of $N$ and $M$ might look something like the step functions sketched in Fig. 2. Realizations of $N$ fluctuate up and down about a time-independent mean value, whereas realizations of $M$ are monotone and nondecreasing. Observe that changes in $M$ are limited to unit steps; decreases in $N$ are likewise limited to unit steps, but increases in $N$ can be integer steps greater than one because more than two neutrons can be emitted in a fission.

\section{Probability Mass Flow Paths}

Our notation and assumptions are established. Turn now to the task of finding a family of ordinary differential equations satisfied by the joint probability mass function (JPMF) that describes $N(t)$ and $M(t)$. To that end, let

$$
\left.P_{1, m}(t)=P \mid N(t)=n \cap M(t)=m\right]=\text { the probability that } N(t) a_{n} \approx n \text {. ses the value } n \text { and } M(t)
$$
assumes the value $m$. Variables $n$ and $m$ range over the non negative integers.

$P_{n, m}(t)$ is the JPMF that describes $N(t)$ and $M(t)$. To find the differential equations satisfied by $P_{n, m}$ we set up a balance relation. Consider Fig. 3, which represents a portion of the state space in
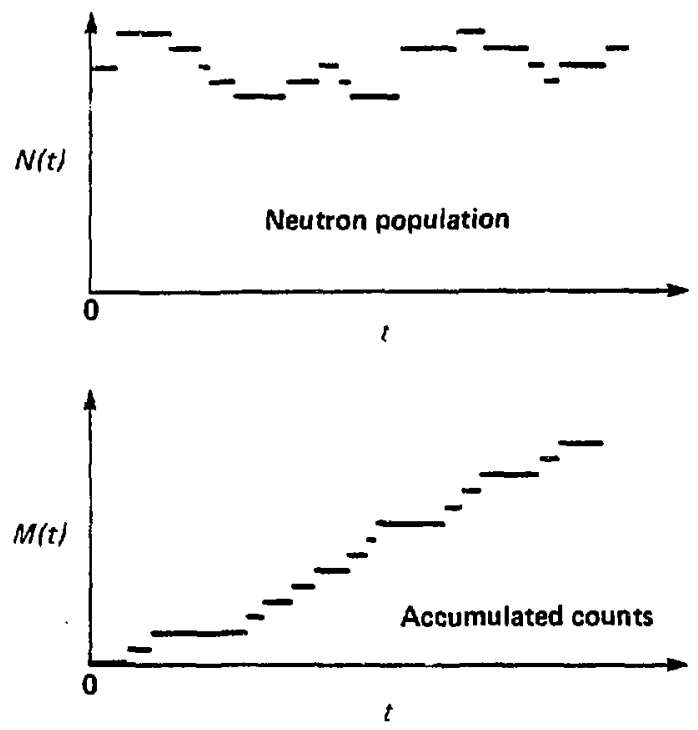

Fig. 2. Schematic of possible realizations of neutron population and counter sample paths. Scales are arbitrary. 


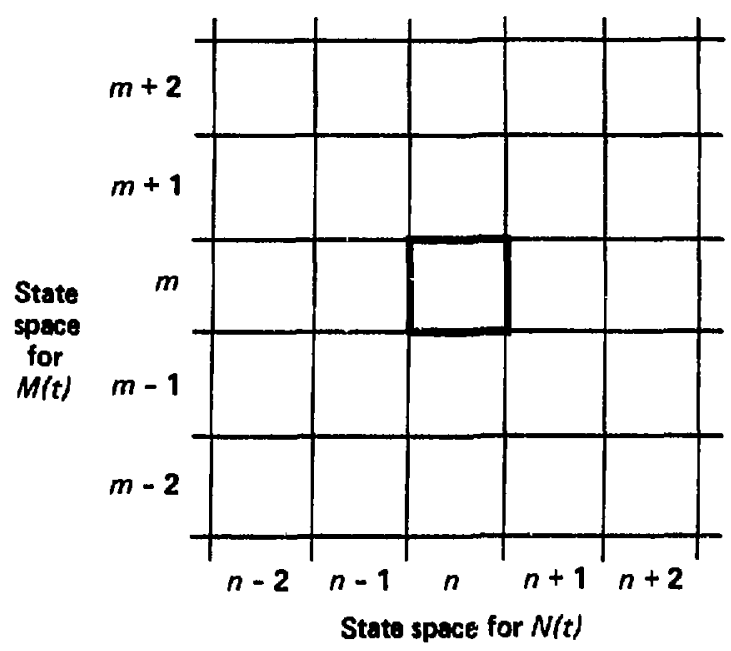

Fig. 3. Sketch of a portion of the state space for the random vector $(N(t), M(t))$.

which $N(t)$ and $M(t)$ assume values. The cell bordered in bold lines is the $(n, m)$ cell. The idea of the derivation is to write down a rate equation that shows how "probability mass" flows into and out of the $(n, m)$ cell. In a small increment of time the state can change by a source emission, a parasitic capture or nondetected leakage, a fission, an extraneous background count, or a detection.

\section{Outflow}

First, consider how the state can change from $(n, m)$ to other states, i.e., how probability can flow from the $(n, m)$ cell. Let $h$ be a "small" increment of time (eventually I will let $h$ tend to 0 ). Figure 4, an enlarged version of the state-space picture, shows the possible paths that lead from the $(n, m)$ staie to neighboring states.

We now attempt to assign probabilities to the paths indicated in Fig. 4 . I give a detailed discussion of path 1 and an abbreviated discussion of the others.

Arrow 1 represents a transition from $(n, m)$ to $(n-1, m)$ via parasitic capture or nondetected leakage. Let

$P[1]$ - probability of a transition from $(n, m)$ to $(n-1, m)$ via path 1 in time interval $[t, t+h]$,
where $h$ is small. In expanded notation the probability we seek is

$P\{1\}=P(N(t+h)=n-1 \cap M(t+h)=m \cap N(t)=n \cap M(t)=m]$.

The set intersection symbol, $\cap$, may be read as "and." In preparation for assigning a value to $P[1]$, decompose the right side of Eq. (3) as follows:

$$
\begin{aligned}
P[1]=P[N(t+h)=n & -1 \cap M(t+h)=m \mid N(t)=n \cap M(t)=m] \\
& \times P[N(t)=n \cap M(t)=m] .
\end{aligned}
$$




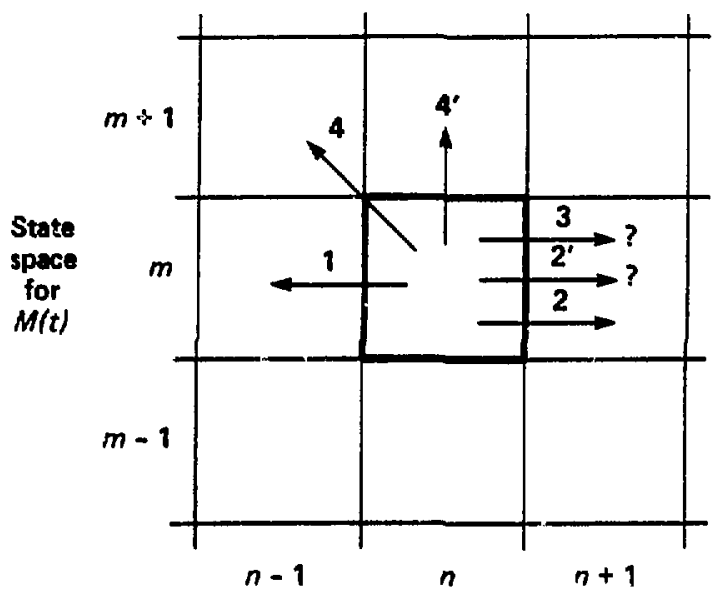

State space for $N(t)$

Fig. 4. Possible transition paths from the $(n, m)$ state. 1 is parasitic capture or nondetected leakage, 2 is emission of a neutron by the rionfission source, 2 ' is fission of a fission-source nucleus, 3 is fission of a fuel nucleus, 4 is detection of a leaked neutron from the assembly, 4 ' is detection of a neutron from an extraneous source (background count). The question marks at the tips of arrow 2 ' and 3 are reminders that the position of the final state depends upon the number of neutrons emitted in a fission.

In writing Eq. (4) I have invoked the definition of conditional probability,

$P \mid A \cap B\}=P\{A \mid B\} P[B]$,

with

$A=\{N(t+h)=n-1 \cap M(t+h)=m\}$.

$B=\{N(t)=n \cap M(t)=m\}$.

The vertical bar used in Eqs. (4) and (5) may be read as "given that." We now assume that

$$
P[N(t+h)=n-1 \cap M(t+h)=m \mid N(l)=n \cap M(t)=m]=n \frac{h}{\tau_{C}}+o(h)
$$

for small $h$. In writing Eq. (8) we are assuming that the probability of a transition in a small time increment $h$ is proportional to $h$ and to the mean transition rate. The symbol $o(h)$ is the usual one and reads "little oh $h$. ." The symbol is a place holder for a function ( $f$, say) with the property that

$\lim _{h \rightarrow 0} \frac{f(h)}{h}=0$.

The o symbol is generic and may represent different functions at different appearances, but it always represents some function with the property shown in Eq. (9).

Bring in the notation introduced above:

$P_{n, m}(t)=P[N(t)=n \cap M(t)=m]$. 
Then Eqs. (10) and (8) into Eq. (4) yields

$P|1|=\left[n \frac{h}{\tau_{C}}+o(h)\right] P_{n, m}(t)$

our final form for $P[1]$. We have completed the task of assigning a probability to path 1 of Fig. 4 .

The arguments for paths $2,2^{\prime}, 3,4$, and $4^{\prime}$ are similar to that used for path 1 , so 1 just write down the results:

$$
\begin{aligned}
& P|2|=\left[\frac{h}{\tau_{\mathrm{RS}}}+v(h)\right] P_{n, m}(t), \\
& P\left|2^{\prime}\right|=\left[\frac{h}{\tau_{\mathrm{FG}}}+v(h)\right] P_{n, m}(t), \\
& P^{\prime}|3|=\left[n \frac{h}{\tau_{1}}+v(h)\right] P_{n, m}(t), \\
& P|4|=\left[n \frac{h}{\tau_{\mathrm{M}}}+v(h)\right] P_{n, m}(t), \\
& P\left|4^{\prime}\right|=\left[\frac{h}{\tau_{\mathrm{MB}}}+v(h)\right] P_{n, m}(t) .
\end{aligned}
$$

Equations (12) th-nugh (16) assign probabilities to nonfission source emission, fission of a fissionsoutce nucleus, fission of a fuel nucleus, detection of a neutron that leaked from the assembly, and detection of an extraneous neutron, respectively. Equations (13) and (14) each overstate the transition probability from state $(n, m)$, since it is possible for a fuel fission to emit one prompt neutron and a spontaneous-source fission to emit zero neutrons. This overstatement will be compensated for when we look at transitions into state $(n, m)$ from the neighboring states, the subject to which ive now turn.

\section{Inflow}

Figure 5 shows possible paths into state $\{n, m\rangle$ from neighboring states. Arguments similar to that used above to derive Eq. (11) lead to the following:

$$
\begin{aligned}
& P[5]=\left[\frac{h}{\tau_{\mathrm{RS}}}+o(h)\right] P_{n-1, m}(t), \\
& F[6]=\left[(n+1) \frac{h}{\tau_{\mathrm{M}}}+o(h)\right] P_{n+1, m-1}(t), \\
& P[7]=\left[(n+1) \frac{h}{\tau_{\mathrm{C}}}+o(h)\right] P_{n+1, m}(t), \\
& P[9]=\left[\frac{h}{\tau_{\mathrm{MB}}}+o(h)\right] P_{n, m-1}(t) .
\end{aligned}
$$

Equations (17) through (20) assign probabilities to nonfission source emission, detection of a neutron that leaked from the assembly, parasitic capture or nondetected leakage, and detection of an exiraneous neutron, respectively. Assignment of probabilities to paths $5^{\prime}$ and 8 of Fig. 5 requires elaboration. 


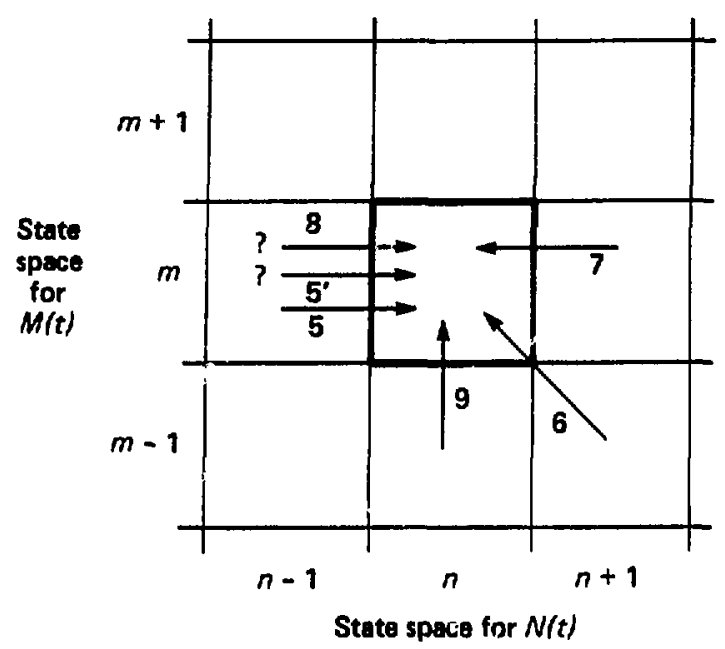

Fig. 5. Possible transition paths into the $(n, m)$ state. 5 is emission of a neutron by the non fission source, 5 ' is fission of a fission-source nucleus, 6 is detection of a leaked neutron from the assembly, 7 is parasitic capture or nondetected leakage, 8 is fission of a fuel nucleus, 9 is detection of a neutron from an extraneous source (background count). The question marks at the tails of arrows 5 and 8 are reminders that the position of the initial state depends upon the number of neutrons emitted in a fission.

The location of the tail of arrow 8, Fig. 5, Jepends upon the number of neutrons emitted in the fission that gave rise to the transition. Suppose the number emitted is $v^{\prime}$. Then transfer is from state $\left(n+1-v^{\prime}, m\right)$ to $(n, m)$. Notice that, if $v^{\prime}=0$, arrow 8 in Fig. 5 points in the wrong direction. So,

$\left(\begin{array}{c}\text { Probability of a transition } \\ \text { from }\left(n+1-v^{\prime} . m\right) \text { to }(n . m)\end{array}\right)=p_{v^{\prime}\left(v^{\prime}\right)}\left[\left(n+1-v^{\prime}\right) \frac{h}{\tau_{\mathrm{F}}}+o(h)\right] P_{n+1-v^{\prime} . m}(f)$

Recall that $\mathrm{p}_{v}\left(v^{\prime}\right)$ is the probability that a fuel fission emits $v^{\prime}$ neutrons. To tind the probability that a fission causes a transfer into $(n, m)$, we must sum over $v$ ' trom 0 to $n$ :

$P[8]=\sum_{v^{\prime}=0}^{n}\left[\left(n+1-v^{\prime}\right) \frac{h}{r_{F}}+v(h)\right] p_{v^{\prime}}\left(v^{\prime}\right) P_{l n+1-v^{\prime}, m}(t)$

The range of summation in Eq. (22) covers all possible states from which transitions can occur into $(n, m)$. Those states run from $(1, m)$ to $(n+1, m)$.

An argument similar to that used to assign probability to arrow 8 leads to the following probability for arrow 5 :

$P[5 ']=\sum_{\mu^{\prime}=0}^{n}\left[\frac{h}{\tau_{\mathrm{FS}}}+o(h)\right] p_{\mu}\left(\mu^{\prime}\right) P_{n-\mu^{\prime}, m}(t)$.

I promised earlier to correct for overstating, in Eqs. (13) and (14), the transition probability from state $(n, m)$. The $v^{\prime}=1$ and $\mu^{\prime}=0$ terms in Eqs. (22) and (23) constitute that correction. 


\section{Differential Equations Satisfied by Joint Probability Mass Function}

Finally we have the machinery available to derive the ordinary differential equation that $P_{n, m}(t)$ satisfies. There exists one such equation for each pair $(n, m)$, where $0 \leq n<\infty$ and $0 \leq m<\infty$. The approach is classic whenever differential calculus is used to construct models of physical situations: cinstruct a certain Newton quotient, then pass to the limit as the denominator tends to zero.

I will need a technical result derivable from probability theory. Recall our underlying probability space $(\Omega, \therefore, P)$. Let $A \in \%^{\prime}$ and $B \in \%^{-}$be two generic events and let $A^{\prime}$ and $B^{c}$ be their comple. ments. Observe that

$$
\begin{aligned}
& i^{3}|A|-P|B| \stackrel{!}{=} P[A \cap \Omega|-P| B \cap \Omega \mid \\
& \stackrel{2}{=} P\left|A \cap\left(B \cup B^{\prime}\right)\right|-P\left|B \cap\left(A \cup A^{c}\right)\right| \\
& \stackrel{3}{=} P\left|(A \cap B) \cup\left(A \cap B^{\circ}\right)\right|-P\left|(B \cap A) \cup\left(B \cap A^{(}\right)\right| \\
& \stackrel{\perp}{=} P|A \cap B|+P\left|A \cap B^{c}\right|-P\left[B \cap A|-P| B \cap A^{*}\right] \\
& \stackrel{\lessgtr}{=} P\left[A \cap B^{\prime} \mid-P\left[B \cap A^{\top}\right] .\right.
\end{aligned}
$$

The reasons:

1. $A$ and $B$ are subsets of the sample space $\Omega$.

2. Clear.

3. Intersection distributes over union.

4. $A \cap B$ and $A \cap B^{\prime}$ are disjoint, as are $B \cap A$ and $B \cap A^{\circ}$, and $P$ is additive on disjoint sets.

5. Intersection is commutative.

Equation (24) is the needed technical result. nition:

The Newton yuotient I wish to construct appears on the right-hand side of the following defi-

$\frac{d}{d t} P_{11, m}(t)=\lim _{h \rightarrow 0} \frac{1}{h}\left[P_{11, m}(t+h)-P_{1, m}(t)\right]$

Ti) prepare for that construction, let $A$ and $B$ represent the following sets;

$A=\left\{\omega \in \Omega: N_{\omega}(t+h)=n\right\} \cap\left\{\omega \in \Omega: M_{\omega}(t+h)=m\right\}$,

$B=\left\{\omega \in \Omega: N_{\omega}(t)=n\right\} \cap\left\{\omega \in \Omega: M_{\omega}(t)=m\right\}$.

In Eys. (26.1) and (26.2) $\omega$ is an element of the sample space (ensemble) $\Omega$, and I have explicitly shown the dependence on $\omega$ of the stochastic processes $N$ and $M$. In the abbreviated notation we have been using above,

$$
\begin{aligned}
& A=[N(t+h)=n \cap M(t+h)=m], \\
& B=[N(t)=n \cap M(t)=m] .
\end{aligned}
$$


Observe that $P_{n, m}\left(t+h_{i}\right)=P[A]$ and $P_{n, m}(t)=P[B]$. In view of Eqs. (24), (26.1'), and (26.2') we have

$$
\begin{aligned}
& P_{n, m}(t+h)-P_{n, M H}(t)=P[A]-P\left[B !=P\left[A \cap B^{c}\right]-P\left[P \cap A^{\mathrm{c}}\right]\right. \\
& =P\left\{[N(t+h)=n \cap M(t+h)=m] \cap[N(t)=n \cap M(t)=m]^{\mathrm{c}}\right\} \\
& \quad-P\left\{[N(t)=n \cap M(t)=m] \cap[N(t+h)=n \cap M(t+h)=m]^{\mathrm{c}}\right\} .
\end{aligned}
$$

In words, Eq. (27) says

$$
\begin{aligned}
& \left(\begin{array}{c}
\text { probability of s.ate } \\
(n, m) \text { at } t+h
\end{array}\right)-\left(\begin{array}{c}
\text { probability of state } \\
(n, m) \text { at } t
\end{array}\right) \\
& =\left(\begin{array}{c}
\text { probability of state }(n, m) \text { at } f+h \\
\text { and not state }(n, m) \text { at } t
\end{array}\right)-\left(\begin{array}{c}
\text { probability of state }(n, m) \text { at } t \\
\text { and not state }(n, m) \text { at } f+h
\end{array}\right) .
\end{aligned}
$$

If we make one more assumption, we have expressions for all terms in Eq. (27) or Eq. (28). Assume that as $h \rightarrow 0$ one and only one of the following can occur:

The system state remains unchanged.

A single fuel nucleus fiss:uns.

A single neutron from the assembly is detected by the M-counter.

A single neutron is lost from the assembly by parasitic capture or nondetected leakage.

A single neutron is emitted from the nonfission source.

A single fission-source nucleus fissions.

A single extraneous neutron is detected by the M-counter.

Somewhat more precisely, we assume that the conjunction of two or more of the above events has probability o( $h$ ) for small $h$. Invoking that assumption, we have

$$
\begin{aligned}
P_{11, m}(t+h)-P_{11, m}(t) & =\left(P[5]+P\left[5^{\prime}\right]+P[6]+P[7]+P[8]+P[9]\right) \\
& -\left(P[1]+P[2]+P\left[2^{\prime}\right]+P[3]+P[4]+P\left[4^{\prime}\right]\right)+v(h) .
\end{aligned}
$$

Now, put Eys. (11) through (20) and Eqs. (22) and (23) intu Eq. (29), divide both sides by $h$, take the limit as $h \rightarrow 0$, and suppress the time argument to obtain

$$
\begin{aligned}
\frac{d}{d t} P_{n, m}= & -n\left(\frac{1}{\tau_{\mathrm{C}}}+\frac{1}{\tau_{\mathrm{F}}}+\frac{1}{\tau_{\mathrm{M}}}\right) P_{n, m}-\left(-\frac{1}{\tau_{\mathrm{RS}}}+\frac{1}{\tau_{\mathrm{F} S}}+\frac{1}{\tau_{\mathrm{MB}}}\right) P_{n, m} \\
& +\frac{(n+1)}{\tau_{\mathrm{C}}} P_{n+1, m}+\frac{(n+1)}{\tau_{\mathrm{M}}} P_{n+1, m-1}+\frac{1}{\tau_{\mathrm{F}}} \sum_{v^{\prime}=0}^{n}\left(n+1-v^{\prime}\right) p_{v^{\prime}}\left(v^{\prime}\right) P_{n+1-v^{\prime}, m} \\
& +\frac{-1}{\tau_{\mathrm{FS}}} \sum_{\mu^{\prime}=0}^{n} p_{\mu}\left(\mu^{\prime}\right) P_{n-\mu^{\prime}, m}+\frac{1}{\tau_{\mathrm{RS}}} P_{n-1, m}+\frac{1}{\tau_{\mathrm{MB}}} P_{n, m-1} ; n \geq 0, m \geq 0 .
\end{aligned}
$$

Finally we have in Eq. (30) the doubly indexed array of ordinary differential equations satisfied by the joint probability mass function that describes the random point $[N(t), M(t)]$. Now, turn the L-counter back on and write down the triply indexed array that describes the random point $[N(t), L(p), M(t)]$. We can do it by trivial extension of Eq. (30). With consistent abuse of notation, let

$P_{n, l, m}(t)=P[N(t)=n \cap L(t)=l \cap M(t)=m]$. 
Then the system of differential equations satisfied by $P_{n, l, m}$ is

$$
\begin{aligned}
\frac{d}{d t} P_{n, l, m}= & -n\left(\frac{1}{\tau_{\mathrm{C}}}+\frac{1}{\tau_{\mathrm{F}}}+\frac{1}{\tau_{\mathrm{L}}}+\frac{1}{\tau_{\mathrm{M}}}\right) P_{n, l, m}-\left(\frac{1}{\tau_{\mathrm{RS}}}+\frac{1}{\tau_{\mathrm{FS}}}+\frac{1}{\tau_{\mathrm{LB}}}+\frac{1}{\tau_{\mathrm{MB}}}\right) P_{n, l, m} \\
& +\frac{(n+1)}{\tau_{\mathrm{C}}} P_{n+1, l, m}+\frac{(n+1)}{\tau_{\mathrm{L}}} P_{n+1,1-1, m}+\frac{(n+1)}{\tau_{\mathrm{M}}} P_{n+1, l, m-1} \\
& +\frac{1}{\tau_{\mathrm{F}}} \sum_{\left.v^{\prime}=1\right)}^{n}\left(n+1-v^{\prime}\right) p_{v}\left(v^{\prime}\right) P_{n+1-v^{\prime}, l, m}+\frac{1}{\tau_{\mathrm{FS}}} \sum_{\mu^{\prime}=0}^{n} p_{\mu}\left(\mu^{\prime}\right) P_{11-\mu, l, m} \\
& +\frac{1}{\tau_{\mathrm{RS}}} P_{n-1, l, m}+\frac{1}{\tau_{1, \mathrm{~B}}} P_{n, l-1, m}+\frac{1}{\tau_{\mathrm{MB}}} P_{n, l, m-1} ; n \geq 0, l \geq 0, m \geq 0 .
\end{aligned}
$$

The first phase of our model construction is complete; system (32) is the grand result. Equip system (32) with initial conditions and solve, and you have a complete description of the firstorder statistics of the three-dimensional random process $(N, L, M)$. Alas, I do not know how to solve that system, so we must be content to solve for various low-order moments, the task to which I now turn.

\section{Partial Differential Equation Satisfied by Probability Generating Function}

The triply infinite system of ordinary differential equations displayed in system (32) will be replaced by a single partial differential equation, an equation satisfied by the probability generating function (PGF) that describes $[N(t), L(t), M(t)]$.

Let

$$
\begin{aligned}
\therefore \quad= & \text { the PGF that describes }\{N(t), L(t), M(t)\}, \\
\therefore= & \text { the PGF that describes } v \text { (the number of neutrons emitted in a fuel fission), } \\
\therefore \mu= & \text { ne PGF that describes } \mu \text { (the number of neutrons emitted when a fission-source } \\
& \text { nucleus fissions). }
\end{aligned}
$$

Then

$$
\begin{aligned}
& \therefore(x, y, z, l)=\left\langle x^{\vee(t)}, y^{L(n)}=\langle(t)\rangle ; \quad(x, y, z) \in \text { unit cube and } t \geq 0\right. \\
& x_{1}(x)=\left\langle x^{\mu}\right\rangle ; x \in[0,1] \\
& s_{\mu}(x)=\left\langle x^{\mu}\right\rangle ; x \in[0,1] .
\end{aligned}
$$

Now, multiply system (32) by $x^{\prime \prime} y^{\prime} z^{\prime \prime \prime}$ and sum over all triples $(n, l, m)$. The task is tedious but relatively straightforward. I forgo grinding out the details here. The result is

$$
\frac{\partial g}{\partial t}=\left(\frac{x-1}{\tau_{\mathrm{RS}}}+\frac{g_{\mu}(x)-1}{\tau_{\mathrm{FS}}}+\frac{y-1}{\tau_{\mathrm{LB}}}+\frac{z-1}{\tau_{\mathrm{MB}}}\right) g+\left(\frac{g_{v}(x)-x}{\tau_{\mathrm{F}}}+\frac{1-x}{\tau_{\mathrm{C}}}+\frac{y-x}{\tau_{\mathrm{L}}}+\frac{z-x}{\tau_{\mathrm{M}}}\right) \frac{\partial g}{\partial x} .
$$




\section{Differential Equation System Satisfied by Selected Moments}

Our next step is to apply various partial derivative operators to Eq. (34) and evaluate at $(1,1,1, t)$ to obtain a system of nine ordinary differential equations satisfied by selected low-order moments. Use subscript notation for partial derivatives: $g_{x}=\partial g / \partial x, g_{x y}=\partial^{2} g / \partial x \partial y$, etc. Observe that:

$$
\begin{array}{ll}
g_{x}(1,1,1, t)=\langle N(t)\rangle & g_{x y}(1,1,1, t)=\langle L(t) N(t)\rangle \\
g_{y}(1,1,1, t)=\langle L(t)\rangle & g_{x z}(1,1,1, t)=\langle M(t) N(t)\rangle \\
g_{z}(1,1,1, t)=\langle M(t)\rangle & g_{y z}(1,1,1, t)=\langle L(t) M(t)\rangle \\
g_{x x}(1,1,1, t)=\langle N(t)(N(t)-1)\rangle & g_{y y}(1,1,1, t)=\langle L(t)(L(t)-1)\rangle \\
& g_{z z}(1,1,1, t)=\langle M(t)(M(t)-1)\rangle .
\end{array}
$$

The partial derivatives to be applied to Eq. (34) are those suggested by Eq. (35). The results of that application will be more compact and informative if we first introduce the characteristic time constant of the assembly. Let $r$ be that time constant. Then

$$
\frac{1}{\tau}=\frac{1}{r_{C}}+\frac{1}{\tau_{L}}+\frac{1}{\tau_{M}}-\frac{\langle v\rangle-1}{\tau_{F}}
$$

Using this definition of $\tau$, recognizing that the time derivative becomes total, and suppressing the time argument in the expectation symbols, we obtain the following results:

$$
\begin{aligned}
& \frac{d}{d t}\langle N\rangle=-\frac{1}{\tau}\langle N\rangle+\frac{1}{\tau_{\mathrm{RS}}}+\frac{\langle\mu\rangle}{\tau_{\mathrm{FS}}} \\
& \frac{d}{d P}\langle L\rangle=\frac{1}{\tau_{L}}\langle N\rangle+\frac{1}{\tau_{L B}} \\
& \frac{d}{d l}\langle M\rangle=\frac{1}{\tau_{M}}\langle N\rangle+\frac{1}{\tau_{M B}} \\
& \frac{d}{d t}\langle N(N-1)\rangle=-\frac{2}{\tau}\langle N(N-1)\rangle+\left[\frac{\langle v(v-1)\rangle}{\tau_{\mathrm{F}}}+2\left(\frac{1}{\tau_{\mathrm{RS}}}+\frac{\langle\mu\rangle}{\tau_{\mathrm{FS}}}\right)\right]\langle N\rangle+\frac{\langle\mu(\mu-1)\rangle}{\tau_{\mathrm{FS}}} \\
& \frac{d}{d t}\langle L N\rangle=-\frac{1}{\tau}\langle L N\rangle+\frac{1}{\tau_{L}}\langle N(N-1)\rangle+\left(\frac{1}{\tau_{R S}}+\frac{\langle\mu\rangle}{\tau_{\mathrm{FS}}}\right)\langle L\rangle+\frac{1}{\tau_{\mathrm{LB}}}\langle N\rangle \\
& \frac{d}{d t}\langle M N\rangle=-\frac{1}{\tau}\langle M N\rangle+\frac{1}{\tau_{M}}\langle N(N-1)\rangle+\left(\frac{1}{\tau_{\mathrm{RS}}}+\frac{\langle\mu\rangle}{\tau_{\mathrm{FS}}}\right)\langle M\rangle+\frac{1}{\tau_{\mathrm{MB}}}\langle N\rangle \\
& \frac{d}{d t}\langle L M\rangle=\frac{1}{\tau_{\mathrm{LB}}}\langle M\rangle+\frac{1}{\tau_{\mathrm{MB}}}\langle L\rangle+\frac{1}{\tau_{\mathrm{L}}}\langle M N\rangle+\frac{1}{\tau_{\mathrm{M}}}\langle L N\rangle \\
& \frac{d}{d t}\langle L(L-1)\rangle=\frac{2}{\tau_{L}}\langle L N)+\frac{2}{\tau_{L B}}\langle L\rangle \\
& \frac{d}{d t}\langle M(M-1)\rangle=\frac{2}{\tau_{M}}\langle M N\rangle+\frac{2}{\tau_{M B}}\langle M\rangle .
\end{aligned}
$$


After following such a tortuous path, it is comforting, even if required, that we recapture the famous no-delayed-neutrons, point kinetics equation with external source that describes the time behavior of the average neutron population, Eq. (37.1). The sum of the last two terms on the right side of $\mathrm{Eq}$. (37.1) is the mean rate of emission of neutrons by the two sources (nonfission and fission). The assembly decay time, $\tau$, appearing in Eq. (37.1) and defined in Eq. (36), is the usual decay time, in spite of the appearance of the defining equation.

Tu establish this assertion, recall the definitions of $\tau_{C}, \tau_{L}$, and $\tau_{M}$ :

$$
\begin{aligned}
& \frac{n}{\tau_{\mathrm{C}}}=\begin{array}{l}
\text { mean rate of disappearance of neutrons from the assembly by parasitic capture } \\
\text { and nondetected leakage when the neutron population in the assembly is } n \text {. }
\end{array} \\
& \frac{n}{r_{1}}=\begin{array}{l}
\text { mean count rate in the L-counter caused by neutrons leaked from the assembly } \\
\text { when the neutron population in the assembly is } n,
\end{array} \\
& \frac{n}{\tau_{M}}=\begin{array}{l}
\text { mean count rate in the M-counter caused by neutrons leaked from the assembly } \\
\text { when the neutron population in the assembly is } n .
\end{array}
\end{aligned}
$$

Observe from these definitions and from $\mathrm{Eq}$. (36) that $\tau$ is a characteristic of the assembly, independent of $\tau_{L}$ and $r_{M}$. For a given assembly, the parameter $\tau_{C}$ changes as $\tau_{L}$ and $\tau_{M}$ change such that $1 / \tau_{\mathrm{C}}+1 / \tau_{\mathrm{L}}+1 / \tau_{\mathrm{M}}$ remains constant. For example, consider the following two cases: (1) the L-counter and $M$-counter are turned on, and (2) the L-counter is turned on, but the $M$-counter is turned off. The geometry is fixed. Parameter $\tau_{C}$ in case (1) is larger than in case (2) because in case (1) some leaked neutrons are counted by the $M$-counter, whereas in case (2) none are counted by the M-counter.

Equations (37.2) and (37.3) are, if not familiar, at least easily interpretable. Interpretation of the remaining six members is not so clear.

\section{Dimensionlass Version}

In preparation for solving system (37), it is useful to render that system dimensionless. The natural time unit is . . Hence, make the following replacements for the dependent variables:

$\begin{array}{ll}\langle N(t)\rangle & -x_{1}(t / \tau) \\ \langle L(t)\rangle & -x_{2}(t / \tau) \\ \langle M(t)\rangle & -x_{3}(t / \tau) \\ \langle N(t)(N(t)-1)\rangle & -x_{4}(t / \tau) \\ \langle L(t) N(t)\rangle & -x_{5}(t / \tau) \\ \langle M(t) N(t)\rangle & -x_{6}(t / \tau) \\ \langle L(t) M(t)\rangle & -x_{7}(t / \tau) \\ \langle L(t)(L(t)-1)\rangle & -x_{8}(t / \tau) \\ \langle M(t)(M(t)-1)\rangle & -x_{9}(t / \tau) .\end{array}$


In addition, make replacements for the independent time variable and characteristic times of the assembly as follows:

$\| \tau-\theta$

$\tau_{R S} / \tau-\theta_{R S}$

$\tau_{\mathrm{FS}} / \tau-\theta_{\mathrm{FS}}$

$\tau_{L B} / \tau-\theta_{L B}$

$\tau_{\mathrm{MB}} / \tau-\theta_{\mathrm{MB}}$

$r / \tau-\theta_{F}$

$r_{L} / \tau-\theta_{L}$

$\tau_{M} / \tau-\theta_{M}$

$\frac{1}{\theta_{\mathrm{RS}}}+\frac{\langle\mu\rangle}{\theta_{\mathrm{FS}}}-\frac{1}{\theta_{\mathrm{S}}}$

$\frac{(v(v-1))}{\theta_{F}}-\frac{1}{\bar{\theta}_{F}}$

$\frac{\langle\mu(\mu-1)\rangle}{\theta_{\mathrm{FS}}}-\frac{1}{\bar{\theta}_{\mathrm{FS}}}$

Replacements (38) and (39) into system (37) yield the vector differential equation

$\frac{d x}{d \theta}=A x+c$

where

$x=\left|x_{1}, x_{2}, \ldots, x_{y}\right|^{\prime}$.

$\mathbf{c}=\left\{1 / \theta_{\mathrm{S}}, 1 / \theta_{\mathrm{LB}}, 1 / \theta_{\mathrm{MB}}, 1 / \bar{\theta}_{\mathrm{FS}}, 0, \ldots, 0\right\}^{\mathrm{T}}$,

and the matrix $A$ is displayed in Fig. 6.

\section{Solution}

A formal solution of $\mathrm{Eq} .(40)$ is

$\mathbf{x}(\theta)=\mathrm{e}^{\wedge\left(\theta-\theta_{4}\right)} \mathbf{a}+\int_{\theta_{0}}^{\theta} \mathrm{e}^{\wedge(\theta-s)} \mathrm{c} d s$,

where $\alpha$ is the state vector at time $\theta_{0}$.

In fact, Eq. (41) is more than a formal solution. Matrix representatives of the exponential operaturs can be determined and the indicated matrix multiplication and integrations can be performed. However, $\mathbf{A}$ is sufficiently sparse to make step-by-step solution, one dimension at a time, easier than solution by the method suggested by Eq. (41). 


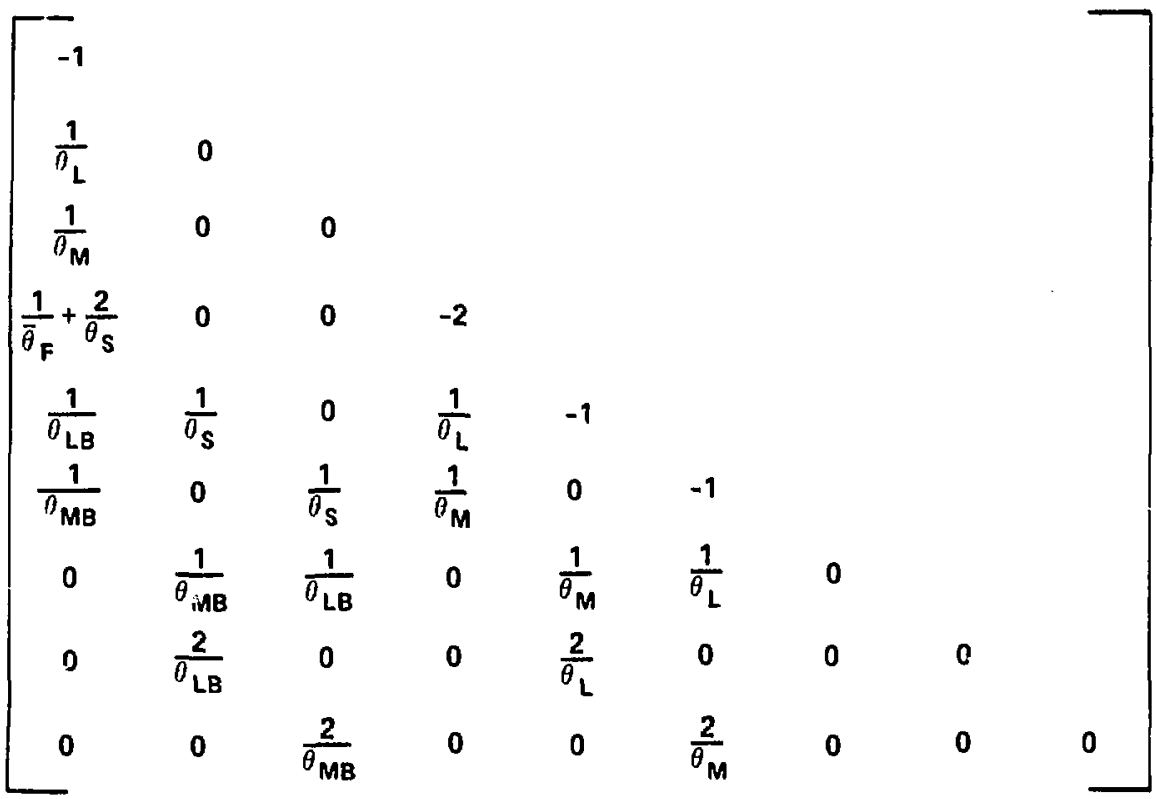

Fig. 6. Matrix A that appears in Eq. (40). A is lower triangular.

I am interested in the "steady-state" solution of Eq. (40), steady-state in the sense that $d x_{1} / \mathrm{d} \theta$ $=d x_{4} i t \theta=0$, the state in which the pure neutron population moments are constant. Invoking the steady-state requirement leads to immediate solutions for $x_{1}$ and $x_{4}$. Further, assume that up to time zero both the $L$ - and $M$-counters are turned off. Hence, at $\theta_{0}=0$ all coordinates of $x$ equal zero except $x_{1}$ and $x_{4}$.

The steady-state requirement and the zero starting condition lead to the following solution of Ey. (40):

$$
\begin{aligned}
& x_{1}=\frac{1}{\theta_{\mathrm{S}}} \\
& x_{2}=\left(\frac{1}{\theta_{\mathrm{L}} \theta_{\mathrm{S}}}+\frac{1}{\theta_{\mathrm{LB}}}\right) \theta \\
& x_{3}=\left(\frac{1}{\theta_{\mathrm{M}} \theta_{\mathrm{S}}}+\frac{1}{\theta_{\mathrm{MB}}}\right) \theta \\
& x_{4}=\frac{1}{2}\left[\frac{2}{\theta_{\mathrm{S}}^{2}}+\left(\frac{1}{\bar{\theta}_{\mathrm{F}} \theta_{\mathrm{S}}}+\frac{1}{\bar{\theta}_{\mathrm{FS}}}\right)\right] \\
& x_{5}=\frac{1}{\theta_{\mathrm{S}}}\left(\frac{1}{\theta_{\mathrm{L}} \theta_{\mathrm{S}}}+\frac{1}{\theta_{\mathrm{LB}}}\right) \theta+\frac{1}{2 \theta_{\mathrm{L}}}\left(\frac{1}{\bar{\theta}_{\mathrm{F}} \theta_{\mathrm{S}}}+\frac{1}{\bar{\theta}_{\mathrm{FS}}}\right)\left(1-\mathrm{e}^{-\theta}\right)
\end{aligned}
$$




$$
\begin{aligned}
& x_{6}=\frac{1}{\theta_{S}}\left(\frac{1}{\theta_{M} \theta_{S}}+\frac{1}{\theta_{M B}}\right) \theta+\frac{1}{2 \theta_{M}}\left(\frac{1}{\bar{\theta}_{\mathrm{F}} \theta_{S}}+\frac{1}{\bar{\theta}_{\mathrm{FS}}}\right)\left(1-e^{-\theta}\right) \\
& x_{7}=\left(\frac{1}{\theta_{\mathrm{L}} \theta_{\mathrm{S}}}+\frac{1}{\theta_{\mathrm{LB}}}\right)\left(\frac{1}{\theta_{\mathrm{M}} \theta_{\mathrm{S}}}+\frac{1}{\theta_{\mathrm{MB}}}\right) \mathrm{e}^{2}+\frac{1}{\theta_{\mathrm{M}}}-\frac{1}{\theta_{\mathrm{L}}}\left(\frac{1}{\bar{\theta}_{\mathrm{F}} \theta_{\mathrm{S}}}+\frac{1}{\bar{\theta}_{\mathrm{FS}}}\right)\left\{\dot{\mu}-\left(1-\mathrm{e}^{-\theta}\right)\right\} \\
& x_{g}=\left(\frac{1}{\theta_{L} \theta_{S}}+\frac{1}{\theta_{L B}}\right)^{2} \theta^{2}+\frac{1}{\theta_{L}^{2}}\left(\frac{1}{\bar{\theta}_{F} \theta_{S}}+\frac{1}{\bar{\theta}_{F S}}\right)\left[\theta-\left(1-\mathrm{e}^{-\theta}\right)\right] \\
& x_{y}=\left(\frac{1}{\theta_{M} \theta_{S}}+\frac{1}{\theta_{M B}}\right)^{2} \theta^{2}+\frac{1}{\theta_{M}^{2}}\left(\frac{1}{\theta_{F} \theta_{S}}+\frac{1}{\theta_{F S}}\right)\left(\theta-\left(1-\mathrm{e}^{-\theta}\right)\right] \text {. }
\end{aligned}
$$

\section{Variance-to-Mean and Covariance-to-Mean Ratios}

Application of some algebraic manipulation will now yield the variance-to-mean and covariance-to-mean ratios. Obserye that, for a generic random variable $(X$, say) with a second moment and a nonzero mean,

$$
\begin{aligned}
\frac{V \mid X]}{\langle x\rangle} & =\frac{1}{\langle x\rangle}\left(\left\langle x^{2}\right\rangle-\langle x\rangle^{2}\right\rangle \\
& =\frac{1}{\langle x\rangle}\left\langle\left\langle x^{2}\right\rangle-\langle x\rangle+\langle X\rangle-\langle X\rangle^{2}\right\rangle \\
& =\frac{1}{\langle x\rangle}\left(\langle X(X-1)\rangle+\langle X\rangle-\langle x\rangle^{2}\right) \\
& =1+\frac{\langle X(X-1)\rangle}{\langle X\rangle}-\langle X\rangle .
\end{aligned}
$$

where $V$ represents the variance operator. Equation (43) and the replacements listed in Eq. (38) suggest that if we desire the variance-to-mean ratio for the L-counter, then we need to compute $v_{\mathrm{L}}(\theta)$, where

$$
\mathrm{i}_{L}(\theta)=1+\frac{x_{1}(\theta)}{s_{2}(\theta)}-x_{2}(\theta)
$$

Equations (42.2) and (42.8) into Eq. (44) yields

$$
\nu_{L}(\theta)=1+\frac{1}{\left(1+\frac{\theta_{L}}{\theta_{L B}} \theta_{S}\right)} \frac{1}{\theta_{L}}\left(\frac{1}{\bar{\theta}_{F}}+\frac{\theta_{S}}{\bar{\theta}_{T_{S} S}}\right) f(\theta),
$$

where

$$
f(\theta)=1-\frac{1-e^{-\theta}}{\theta}
$$

Figure 7 shows a graph of $f$. By symmetry, replace subscript $L$ with $M$ in Eq. (45) and you have an equation for the $M$-counter. 


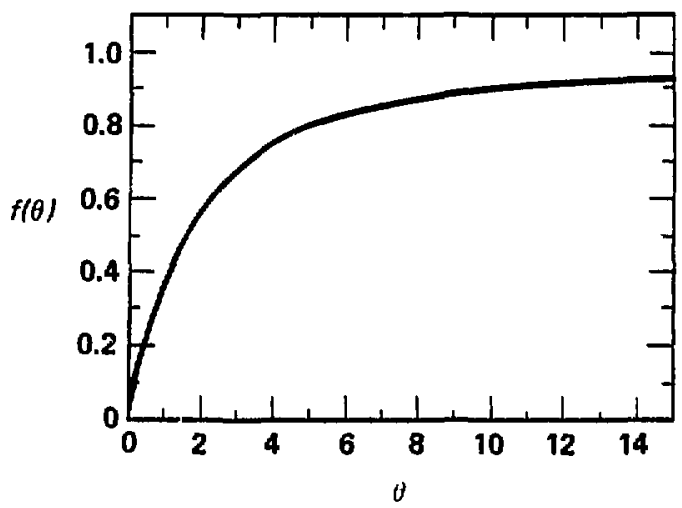

Fig. 7. Graph of function $f$ defined in Eq. (46). Function $f$ is sometimes called the Feynman function.

Now, restore the notation that was abandoned when we adopted dimensionless notation, and Eq. (45) gives $u$, the following equation for the variance-to-mean ratio for the L-counter:

$$
\begin{aligned}
r V m_{\mathrm{L}}(t) & =\frac{V(L(t) \mid}{\langle L(t)} \\
& =1+\frac{1}{1+\frac{\tau_{\mathrm{L}}}{\tau_{\mathrm{LB}}}\left(\frac{1}{\tau_{\mathrm{RS}} / \tau}+\langle\mu\rangle \frac{1}{\tau_{\mathrm{FS}} / \tau}\right)^{-1}} \frac{1}{\tau_{\mathrm{L}} / \tau}\left(\frac{\langle v(\nu-1)\rangle}{\tau_{\mathrm{F}} / \tau}+\frac{\mu(\mu-1)\rangle}{\langle\mu\rangle+\tau_{\mathrm{FS} S} / \tau_{\mathrm{RS}}}\right) f(t / \tau) .
\end{aligned}
$$

Some remarks about Eq. (47) are in order. First, recall that we assumed a spontaneous fission source, a nonfission source, and a nonzero background in deriving Eq. (47). If you turn off the fission source $\left(\tau_{\mathrm{FS}}{ }^{\dagger \infty}\right.$ ) and turn off the background source $\left(\tau_{\mathrm{LB}}{ }^{\dagger \infty}\right)$, E(!. (47) reduces to

$$
i\left(m m_{1}(t)=1+\frac{\langle v(v-1)\rangle}{\left(\tau_{\mathrm{L}} \tau\right)\left(\tau_{\mid} / \tau\right)} f(t \tau)\right.
$$

the first "ine:ttron noise" result ever obtained.' Second, observe from Eq. (47) that, if the background rate is high ( $\tau_{\mathrm{L}} \mathrm{a}$ is small), then the variance-to-mean ratio tends toward 1 , the value for a "pure random" or Poisson process. Finally, notice how the randomness in the number of neutrons emitted in a spontaneous fission increases the variance-to-mean ratio via the term containing $\langle\mu(\mu-1)\rangle$. nition

To find the cross covariance-to-mean ratio between the L- and M-counters, start with the defi-

$c t m(t)=\frac{\langle L(t) M(t)\rangle-\langle L(t)\rangle\langle M(t)\rangle}{(L(t)\rangle^{1 / 2}\langle M(t))^{1 / 2}}$

This definition suggests that we compute $c_{\mathrm{LM}}(\theta)$, where

$$
c_{\operatorname{LM}}(\theta)=\frac{x_{7}(\theta)-x_{2}(\theta) x_{3}(\theta)}{x_{2}(\theta)^{1 / 2} x_{3}(\theta)^{1 / 2}}
$$


Equations (42.2), (42.3), and (42.7) into Eq. (50) yields

$$
c_{\mathrm{LM}}(\theta)=\frac{1}{\left(1+\frac{\theta_{\mathrm{L}} \theta_{\mathrm{S}}}{\theta_{\mathrm{LB}}}\right)^{1 / 2}\left(1+\frac{\theta_{\mathrm{M}} \theta_{\mathrm{S}}}{\theta_{\mathrm{MB}}}\right)^{1 / 2}} \frac{1}{\theta_{\mathrm{L}}^{1 / 2} \theta_{\mathrm{M}}^{1 / 2}}\left(\frac{1}{\overline{\bar{\theta}}_{\mathrm{F}}}+\frac{\theta_{\mathrm{S}}}{\bar{\theta}_{\mathrm{FS}}}\right) f(\theta) .
$$

Restore the primitive notation and obtain

$$
\begin{gathered}
c t m(l)=\frac{1}{\left[1+\frac{\tau_{\mathrm{L}}}{\tau_{\mathrm{LB}}}\left(\frac{1}{\tau_{\mathrm{RS}} / \tau}+\langle\mu\rangle \frac{1}{\tau_{\mathrm{FS}} / \tau}\right)^{-1}\right]^{1 / 2}} \frac{1}{\left[1+\frac{\tau_{\mathrm{M}}}{\tau_{\mathrm{MB}}}\left(\frac{1}{\tau_{\mathrm{RS}} / \tau}+\langle\mu\rangle \frac{1}{\tau_{\mathrm{FS}} / \tau}\right)^{-1}\right]^{1 / 2}} \\
\quad \times \frac{1}{\left(\tau_{\mathrm{L}} / \tau\right)^{1 / 2}\left(\tau_{\mathrm{M}} / \tau\right)^{1 / 2}}\left(\frac{\langle\nu(\nu-1)\rangle}{\tau_{\mathrm{F}} / \tau}+\frac{\langle\mu(\mu-1)\rangle}{\langle\mu\rangle+\tau_{\mathrm{FS}} / \tau_{\mathrm{RS}}}\right) f(N / \tau\rangle .
\end{gathered}
$$

Except for the added 1 in Eq, (47), Eqs. (47) and (52) are similar; taking the cross covariance suppresses the 1 , which is the variance-to-mean ratio for a Poisson process. Observe that the extraneous background pushes the covariance-to-mean ratio toward 0 just as it pushes the variance-tomean ratio toward 1 .

\section{Serial-Covariance-to-Mean Ratio}

So far we have employed the model to find the variance-to-mean ratio of the number of counts in a time bin vs time bin width and the covariance-to-mean ratio of the numbers of counts accumulated in a time bin by two separate counters vs time bin width. It turns out that we can find the covariance-to-inean ratio of counts accumulated in two sequential, nonintersecting time bins by exploiting the stationarity of a process closely related to the counting process (which is not stationary). I now do that.

First, I state the result. Pick times $0<t_{1}<t_{2}$ and $h>0$ such that the time intervals $\left[t_{1}, t_{1}+h\right]$ and $\left[t_{2}, t_{2}+h j\right.$ intersect, if at all, at only one point. Then, within the context of our probabilistic model, the following assertion is true:

$$
\frac{\operatorname{Cov}\left[L\left(t_{1}+h\right)-L\left(t_{1}\right) \cdot L\left(t_{2}+h\right)-L\left(t_{2}\right)\right]}{\langle L(h)\rangle}=d \frac{\cosh (h / \tau)-1}{(h / \tau)} e^{-\frac{\mid t_{2}-t_{1}}{r}},
$$

where Cov represents the covariance operator and

$$
d=\frac{1}{1+\frac{\tau_{\mathrm{L}}}{\tau_{\mathrm{LB}}}\left(\frac{1}{\tau_{\mathrm{RS}} / \tau}+\langle\mu\rangle \frac{1}{\tau_{\mathrm{FS}} / \tau}\right)^{-1}} \frac{1}{\tau_{\mathrm{L}} / \tau}\left(\frac{\langle\nu(\nu-1)\rangle}{\tau_{\mathrm{F}} / \tau}+\frac{\langle\mu(\mu-1)\rangle}{\langle\mu\rangle+\tau_{\mathrm{FS}} / \tau_{\mathrm{RS}}}\right)
$$

Once again, observe that the extraneous background pushes the serial-covariance-to-mean ratio toward 0 just as it pushes the variance-to-mean ratio toward 1 .

'to establish Eq. (53), I need a technical but key result concerning second moments of a stochastic process whose increments are stationary. Let $X(t), 0 \leq t<\infty$ be a stochastic process with stationary increments, i.e., $X(t+h)-X(t)$ is at least second-order stationaly. Assume that $X(0)=0$. Then

$\operatorname{Cov}[X(t), X(t+h)]=\frac{1}{2}\{V[X(t+h)]+V[X(t)]-V[X(h)]\}$ 
To establish Eq. (55), observe that, since Cov is bilinear,

$$
\begin{aligned}
\operatorname{Cov}[X(t), X(t+h)] & =\operatorname{Cov}[X(t), \lambda i t+h)-X(t)+X(t)] \\
& =\operatorname{Cov}[X(t), X(t+h)-X(t)]+\operatorname{Cov}[X(t), X(t)] \\
& =\operatorname{Cov}[X(t), X(t+h)-X(t)]+V[X(t)],
\end{aligned}
$$

and that

$$
\begin{aligned}
V(X(t+h)] & =V\{X(t+h)-X(t)+X(t)] \\
& =V[X(t+h)-X(t)]+V[X(t)]+2 \operatorname{Cov}[X(t+h)-X(t), X(t)] .
\end{aligned}
$$

Now, eliminate $C(v v|X(t), X(t+h)-X(t)|$ between Eqs. (56) and (57) to obtain

$\left.C_{(\mid l} \mid X(t), X(t+h)\right]=\frac{1}{2}\{V \mid X(t+h)]+V[X(t) \mid-V[X(t+h)-X(t)]\}$.

But by hypothesis $X(t)$ has increments that are second-order stationary. Hence,

$V[X(t+h)-X(t)]=V(X(h)-X(0)]=V[X(h)]$.

Substitute Eq. (59) into Eq. (58) and you have the assertion displayed in Eq. (55), the required technical result.

I return to the task of deriving the main result, Eq. (53). First, observe that

$$
\begin{aligned}
\operatorname{Cov} \mid L\left(t_{1}+h\right) & \left.-L\left(t_{1}\right), L\left(t_{2}+h\right)-L\left(t_{2}\right)\right]=\operatorname{Cov}\left[t_{1}\left(t_{1}+h\right), L\left(t_{2}+h\right)\right] \\
& -\operatorname{Cov}\left[I_{(}\left(t_{1}+h\right), L\left(t_{2}\right)\right] \cdot \operatorname{Cov}\left[L\left(t_{1}\right), L\left(t_{2}+h\right)\right]+\operatorname{Cov}\left[L\left(t_{1}\right), L\left(t_{2}\right)\right] .
\end{aligned}
$$

A gain, Eq. (60) follows from the bilinearity of the covariance operator. Now, replace each term on the right side of Eq. (60) with the appropriate version of Eq. (55) to obtain

$$
\begin{aligned}
\operatorname{Cov} \mid L\left(t_{1}+h\right)-L\left(t_{1}\right), L\left(t_{2}+h\right) & \left.-L\left(t_{2}\right)\right]=\frac{1}{2}\left\{V\left[L\left(t_{2}-t_{1}-h\right)\right]\right. \\
& \left.-2 V\left[L\left(t_{2}-t_{1}\right)\right]+V\left[L\left(t_{2}-t_{1}+h\right)\right]\right\} .
\end{aligned}
$$

The next sequence of steps en route to proving Eq. (53) is: (1) multiply Eq. (47) by $\langle L(t)\rangle$, keeping in mind that $\langle L(t)\rangle=x_{2}(t / \tau) ;(2)$ use the result obtained in step (1) to find $\left.\left.V L\left(t_{2}-t_{1}-h\right)\right], V L\left(t_{2}-t_{1}\right)\right]$, and $\left.V L\left(t_{2}-t_{1}+h\right)\right] ;$ (3) plug the results of step (2) into Eq. (61); (4) divide the result of step (3) by $\langle L(h)\rangle$. You arrive at Eq. (53), which I call the serial-covariance-to-mean ratio. Execution of the indicated steps requires some uninteresting algebraic manipulation. I do not display it.

A useful special case of Eq. (53) arises if $t_{2}-t_{1}=h$, the case in which the two time increments are adjacent and of equal length. In that case Eq. (53) becomes

$\frac{C_{i v v}[L(t+h)-L(t), L(t+2 h)-L(t+h)]}{\langle L(h)\rangle}=d w(h / \tau)$,

where

$w(x)=\mathrm{e}^{-x} \frac{\cosh x-1}{x}$.

Figure 8 is a graph of Eq. (63). 


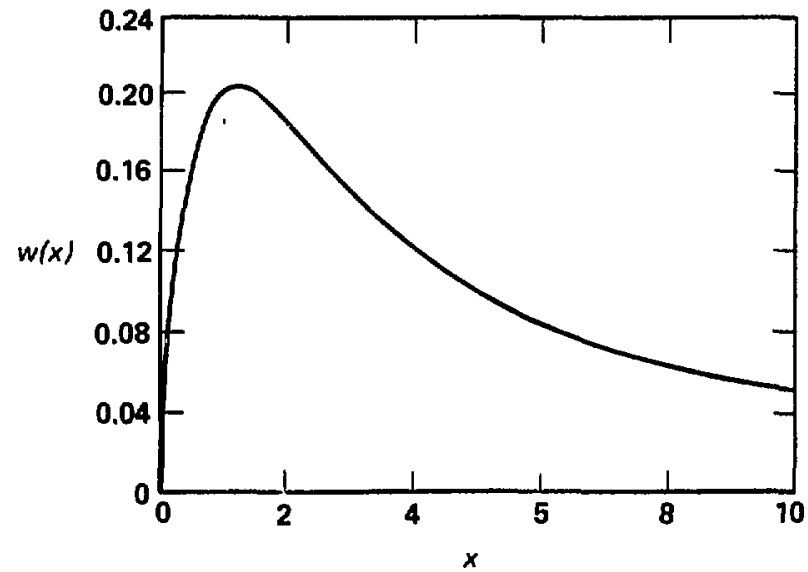

Fig. 8. Graph of function $w$ defined in Eq. (63). 


\section{Measured Discriminator Values}

In this chapter I display the data collected from the multiplying assembly (detailed in the Appendix) so that I can examine the implications of these data in the next chapter. The first and major section of this chapter displays graphs of mean values of discriminator intensities vs two independent variables: assembly fuel mass and calculated assembly multiplication factor. Curves appear for three discriminators: (1) asymptotic increment in the variance-to-mean ratio, (2) asymptotic value of the covariance-to-mean ratio, and (3) coefficient of the serial-covariance-to-mean ratio function.

The second section shows graphs of mean values of estimators of the assembly time constant vs assembly fuel mass. Estimators of assembly time constant were not useful as discriminators.

Many two-parameter least-squares fits of data to theoretical formulae were required to win the discriminator curves from the raw data. The last section displays several examples of such curve fits.

\section{Mean Values of Discriminator Intensities}

\section{Asymptotic Increment in the Variance-to-Mean Ratio}

Figure 9 (a) shows pluss of the asymptotic increment in the variance-to-mean ratio vs fuel mass for three different detection situations. All three curves are the result of measurements taken while the detector bank were in the configuration described in the Appendix. The different curves were obtained by selecting three different subsets of the sixteen detectors for data processing.

I now describe in some detail how the curves were obtained, using as an example the 16detector curve, the top curve. To generate that curve, first the nonfission source and one fuel shell were inserted into the muitiplying assembly. The recording apparatus was turned on and one floppy disk filled with detection time and detector identity data (nominally 120,000 events). The same was done for 19 more disks of data, and the 20 disks of data were stored in the central LLNL computer data bank for processing on the CDC 7600 computers. With software developed for the purpose, I then calculated the variance-to-mean ratio vs time bin width for the data on disk 1 . treating all 16 detectors as one detector. Time bin widths chosen were $250 \mu \mathrm{s}, 500 \mu \mathrm{s}, \ldots, 5000 \mu \mathrm{s}$. With additional specially developed software, I did a two-parameter least-squares fit of the 20 data puints generated, to the formula

$V / M=1+r(1)\left(1-\frac{1-\mathrm{e}^{-1 / r}}{t / r}\right)$.

where

$V / M=$ variance-to-mean ratio,

$x(1)=$ one of the parameters, the asymptotic increment in variance-to-mean ratio,

$t \quad=$ the time bin width,

$\mathrm{r}=$ the other parameter, the assembly decay-time constant.

This step gave me two numbers, $x(1)_{1}$ and $r_{1}$.

Disks 2 through 20 were processed the same way, giving twenty parameter pairs $\left(x(1)_{i}, \tau_{i}\right), 1 \leq i$ $\leq 20$. Calculating the sample mean and sample standard deviation of the $\left\{x(1)_{i}\right\}_{i=1}^{2 r}$,

$\overline{x(1)}=\frac{1}{20} \sum_{i=1}^{20} x(1)_{i}$

$\sigma(x(1))=\left[\frac{1}{20} \sum_{i=1}^{20}\left(x(1)_{i}-\overline{x(1)}\right)^{2}\right]^{1 / 2}$,

produced the left-most point on the 16-detector curve $(5.49, \overline{x(1)})$ and its uncertainty bars. 

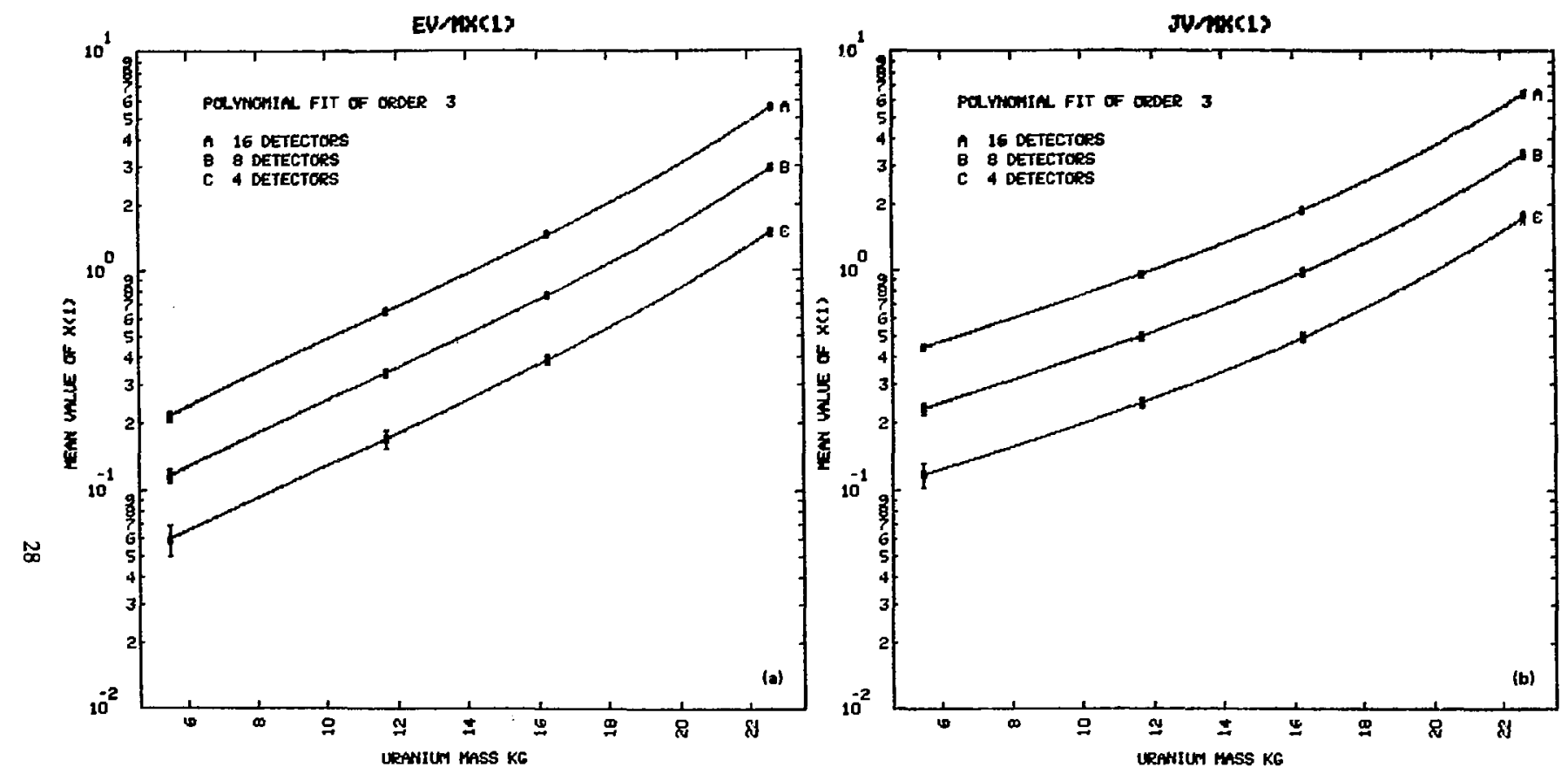

Fig. 9. Asymptotic increment in the variance-to-mean ratio plotted against the uranium mass in the assembly. Each datum is the mean of 20 determinations. Error bars are centered at the mean and are $2 \sigma$ long, where $\sigma$ is the sample standard deviation calculated from the 20 determinations. $A$ full disk of data was processed for each determination. (a) was driven by the nonfission (PuBe) source; (b) was driven by the fission ( ${ }^{252} \mathrm{Cf}$ ) source. 
The same sequence repeated for two, three, and four fuel shells generated the remaining points on the 16-detector curve. The points on the 8-detector curve were generated with the same 80 disks of data that were used to generate the 16 -detector curve. The steps are identical to those used in the 16-detector case except that only the events recorded by detectors $3,4,5,6,11,12,13$, and 14 were used. The remaining detectors were turned "off" by the computer program. The points on the 4-detector curve were generated as in the 8-detfctor case except that only events from detectors $4,5,12$, and 13 were used.

Figure 9(b) is similar to Fig. 9(a) except that the data shown in Fig. $9(b)$ were generated while the assembly was driven with the fission source.

Figure 10 displays the asymptotic increment in the variance-to-mean ratio vs the multiplication factor. The multiplication factor $(k)$ was calculated for each of the four fuel loadings with a deterministic neutron transport code (the SAN code with a 92-group cross section library).

I have just described how a sample of the discriminator $x(1)$, the asymptotic increment in the variance-to-mean ratio, is obtained: by a least-squares fit of 20 variance-to-mean ratio estimates to $E_{y}$. (64), $x(1)$ and $\tau$ serving as parameters. Note that the procedure is equivalent to subtracting 1 from each of the 20 estimates of the variance-to-mean ratio and doing a least-squares fit to $V / M-1$ $=x(1)[1-(1-\exp (-H / \tau)) /(l / \tau) \mid$. The pair $(x(1), \tau)$ that minimizes the deviation in the first case is identical to the pair that minimizes the deviation in the latter case.

\section{Asymptotic Value of the Covariance-to-Mean Ratio}

Figure 11(a) shows plots of the asymptotic covariance-to-mean ratio vs fuel mass for three detector situations, obtained by selecting three different subsets of the set of 16 detectors. The curves were obtained from the same data disks used for the variance-to-mean ratio case. For the top curve I used specially developed software to calculate the covariance-to-mean ratio vs time bin width for the data from disk 1 . Calculation of a covariance-to-mean ratio requires that two detectors be employed, so the 16 detectors were grouped into two sets, the first consisting of the odd-numbered detectors and the second consisting of the even-numbered detectors. Time bin widths chosen were $250 \mu \mathrm{s}, 500 \mu \mathrm{s}, \ldots, 5000 \mu \mathrm{s}$. With software developed for the purpose, I then did a twoparameter least-squares fit of the 20 data points generated, to the formula

$C / M=x(1)\left(1-\frac{1-\mathrm{e}^{-1 / t}}{t / \tau}\right)$.

where

$C / M=$ the cuvariance-to-mean ratio,

$x(1)=$ one of the parameters, the asymptotic covariance-to-mean ratio,

$1=$ the time bin width.

$r \quad=$ the other parameter, the assembly decay-time constant.

This step gave me two numbers: $x(1)_{1}$, and $r_{1}$.

Disks 2 through 20 were processed the same way, giving 20 parameter pairs. Calculating the sample mean and sample standard deviation as for thie variance-to-mean ratio case produced the left-most point on the 16-detector curve $(5.49, \overline{x(1)})$ and its uncertainty bars.

The same sequence repeated for two, three, and four fuel shells generated the remaining points on the 16-detector curve. The points on the 8-detector curve were generated with the same 80 disks of data that were used to generate the 16-detector curve. The steps are identical to thuse used in the 16-detector case except for detector selection. For the 8-detector case, the selection was

Set $\# 1=\{3,5,12,14\}$

Set $\# 2=\{4,6,11,13\}$.

The points on the 4-detector curve were generated as in the 8-detector case except that detector selection was

Set \#1 $=\{4,5\}$

Set $\# 2=\{12,13\}$.

Figure 11(b) is similar to Fig. 11(a) except that the data shown in Fig. 11(b) were generated while the assembly was driven with the fission source. 

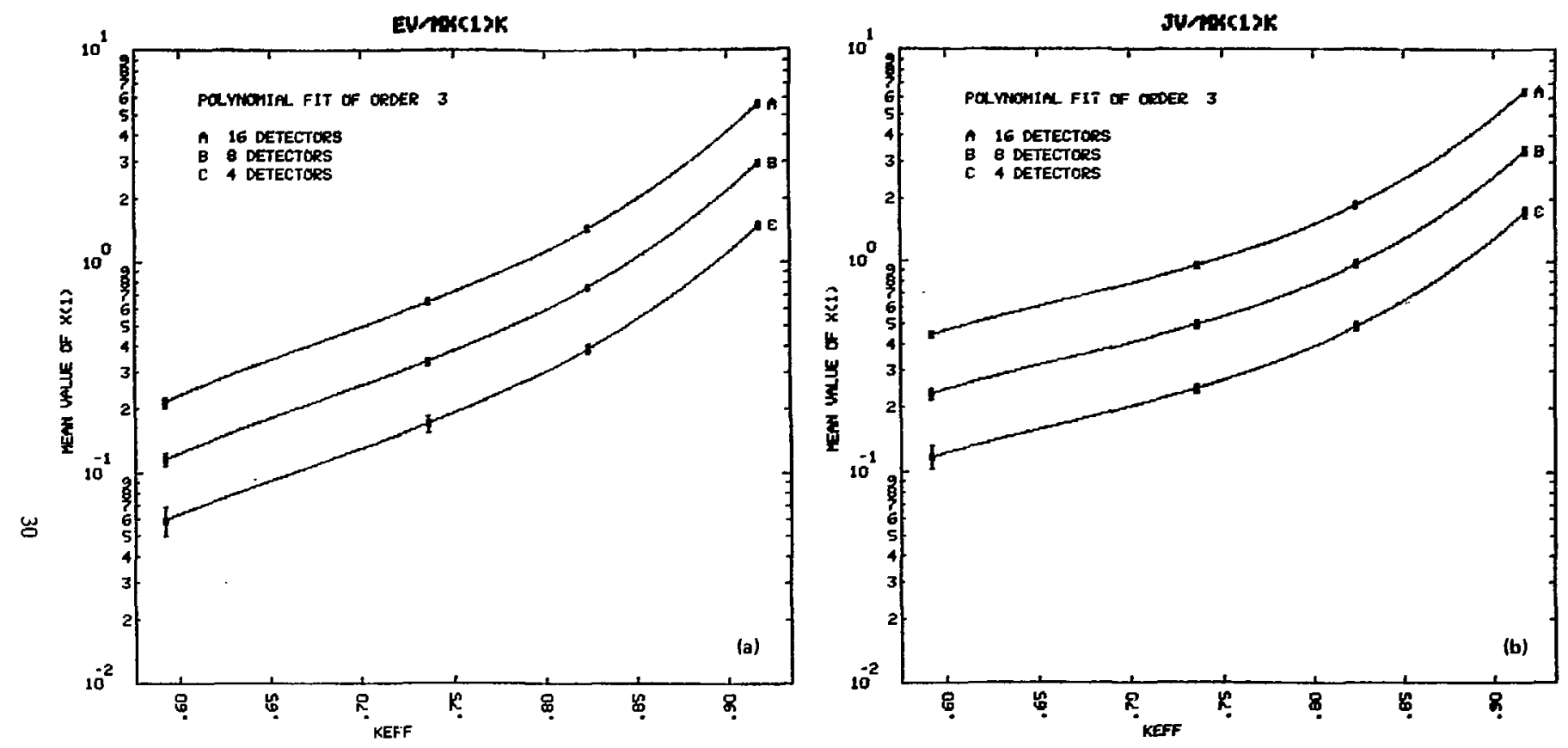

Fig. 10. Asymptotic increment in the variance-to-mean ratio plotted against the calculated multiplication factor $(k)$ of the assembly. Each datum is the mean of 20 determinations. Error bars are centered at the mean and are $2 \pi$ long, where $\sigma$ is the sample standard deviation calculated from the 20 determinations. A full disk of data was processed for each determination. (a) was driven by the nonfission (PuBe) source; (b) was driven by the fission $\left(^{252} \mathrm{Cf}\right)$ source. 

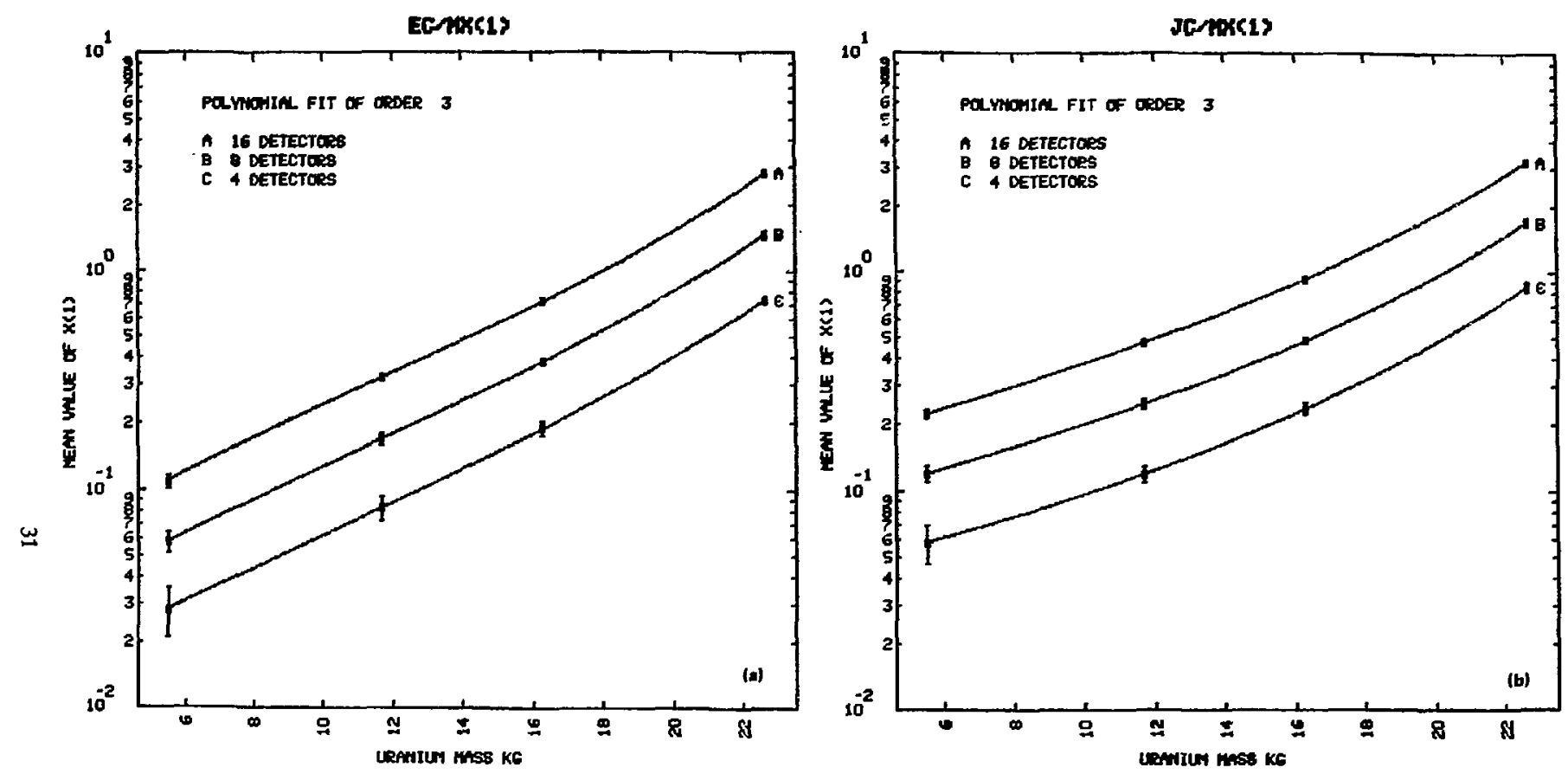

Fig. 11. Asymptotic value of the covariance-to-mean ratio plotted against the uranium mass in the assembly. Each datum is the mean of 20 determinations. Error bars are centered at the mean and are $2 \sigma$ long, where $\sigma$ is the sample standard deviation calculated from the 20 determinations. $A$ full disk of data was processed for each determination. (a) was driven by the nonfission (PuBe) source; (b) was driven by the fission $\left({ }^{25} \mathrm{C} C\right)$ source. 
Figure 12 displays the asymptotic covariance-to-mean ratio vs the multiplication factor curves. The multiplication factor $(k)$ was calculated as described above.

\section{Coefficient of the Serial-Covariance-to-Mean Ratio Function}

Figure 13(a) shows plots of the coefficient vs fuel mass for three different detector situations. The different curves were obtained by selecting three different subsets of the set of 16 detectors, using the same data disks as for the variance-to-mean and covariance-to-mean ratio cases.

To obtain the top curve, I first calculated the covariance-to-mean ratio for counts in adjacent equal-length time bins for the data on disk 1 . All 16 detectors were treated as one detector, and time bin widths were $100 \mu \mathrm{s}, 200 \mu \mathrm{s}, \ldots, 2500 \mu \mathrm{s}$. I then did a two-parameter least-squares fit of the 25 data points generated, to the formula

$$
S C / M=x(1) \mathrm{e}^{-1 / r} \frac{\cosh (t / \tau)-1}{(t / \tau)}
$$

where

$S C I M=$ the covariance-to-mean ratio of counts in adjacent, equal-width time bins,

$x(1)=$ one of the parameters, the coefficient of the serial-covariance-to-mean ratio function,

$1=$ the time bin width,

$\tau \quad=$ the other parameter, the assembly decay-time constant.

This step gave two numbers, $x(1)_{1}$ and $\tau_{7}$. Disks 2 through 20 were processed the same way, giving 20 parameter pairs. Calculating the sample mean and sample standard deviation as for the variance-to-mean ratio case produced the left-most point on the 16-detector curve $(5.49, \overline{x(1)})$ and its uncertainty bars.

The same sequence repeated for two, three, and four fuel shells generated the remaining points on the 16-detector curve. The points on the 8-detector curve were generated with the same 80 disks of data that were used to generate the 16-detector curve. The steps are identical to those used in the 16-detector case except that only the events recorded by detectors $3,4,5,6,11,12,13$, and 14 were used. The points on the 4-detector curve were generated as in the 8-detector case except that only events recorded by detectors $4,5,12$, and 13 were used.

Figure $13(b)$ is similar to Fig. $13(a)$ except that the data shown in Fig. 13(b) were generated while the assembly was driven with the fission source.

Figure 14 displays the coefficient of the serial-covariance-to-mean ratio function vs the multiplication factor. The multiplication factor $(k)$ was calculated as described above.

\section{Influence of Extraneous Background on Discriminators}

Recall that $I$ included provision for extraneous background counts in the probabilistic mudel and that according to the model, extraneous background had a common effect on each of the three discriminators (see Eqs. (47), (52), (54), and the discussions in the neighborhoods of those equations). Figures 15 through 17 demonstrate the background effert.

Each of Figs. 15, 16, and 17 consists of two curves. The upper curve of each figure is a repeat of the upper curve in Figs. 9,11, and 13, respectively. The lower curve of each figure is based on data taken under conditions identical to those that underlie the upper curve, with one exception. Data for each lower curve were taken when the detectors were exposed to neutrons from an extraneous nonfission neutron source. Also, the lower curves were distilled from only 5 disks of data, whereas the upper curves were distilled from 20.

The curves show that qualitatively the various discriminators are weakened by extraneous background roughly as predicted by the theory. To support this assertion for the variance-to-mean ratio case, consider Eq. (47), which displays the theoretical variance-to-mean ratio. The extraneous background enters $\mathrm{Eq}$. (47) through the term that contains $\tau_{\mathrm{LB}}$, the mean time between background counts. Call that term $b$. Then

$b=\frac{1}{1+\frac{1 / \tau_{\mathrm{LB}}}{\left(1 / \tau_{\mathrm{RS}}+(\mu) / \tau_{\mathrm{FS}}\right)\left(\tau / \tau_{\mathrm{L}}\right)}}$. 

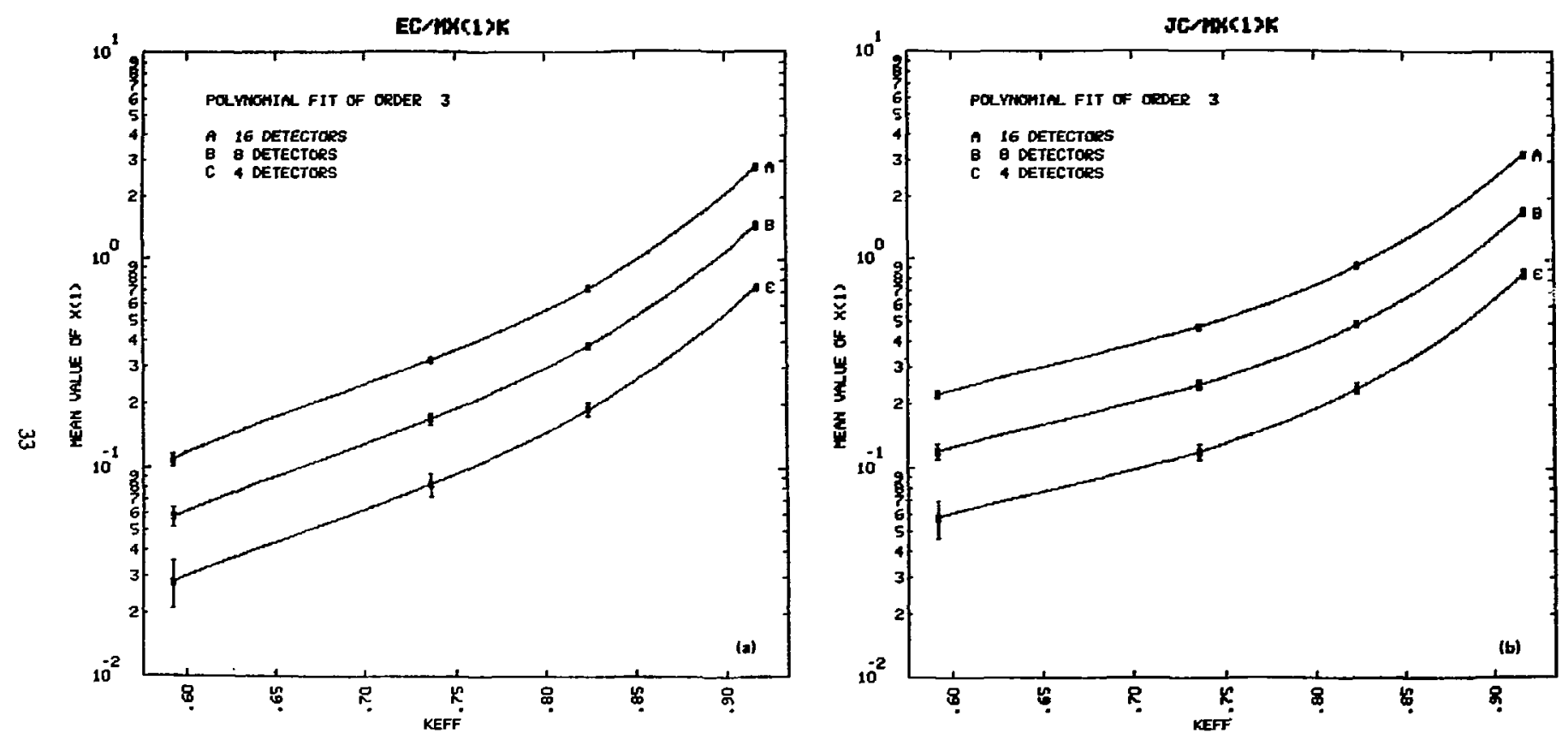

Fig. 12. Asymptotic value of the covariance-to-mean ratio plotted against the calculated multiplication fac or $(k)$ of the assembly. Each datum is the mean of $\mathbf{2 0}$ determinations. Error bars are centered at the mean and are $\mathbf{2 \sigma}$ long, where $\sigma$ is the sainple standard deviation calculated from the $\mathbf{2 0}$ determinations. A full disk of data was processed for each determination. (a) was driven by the nonfission (PuBe) source; (b) was driven by the fission $\left.{ }^{252} \mathrm{Cf}\right)$ source. 

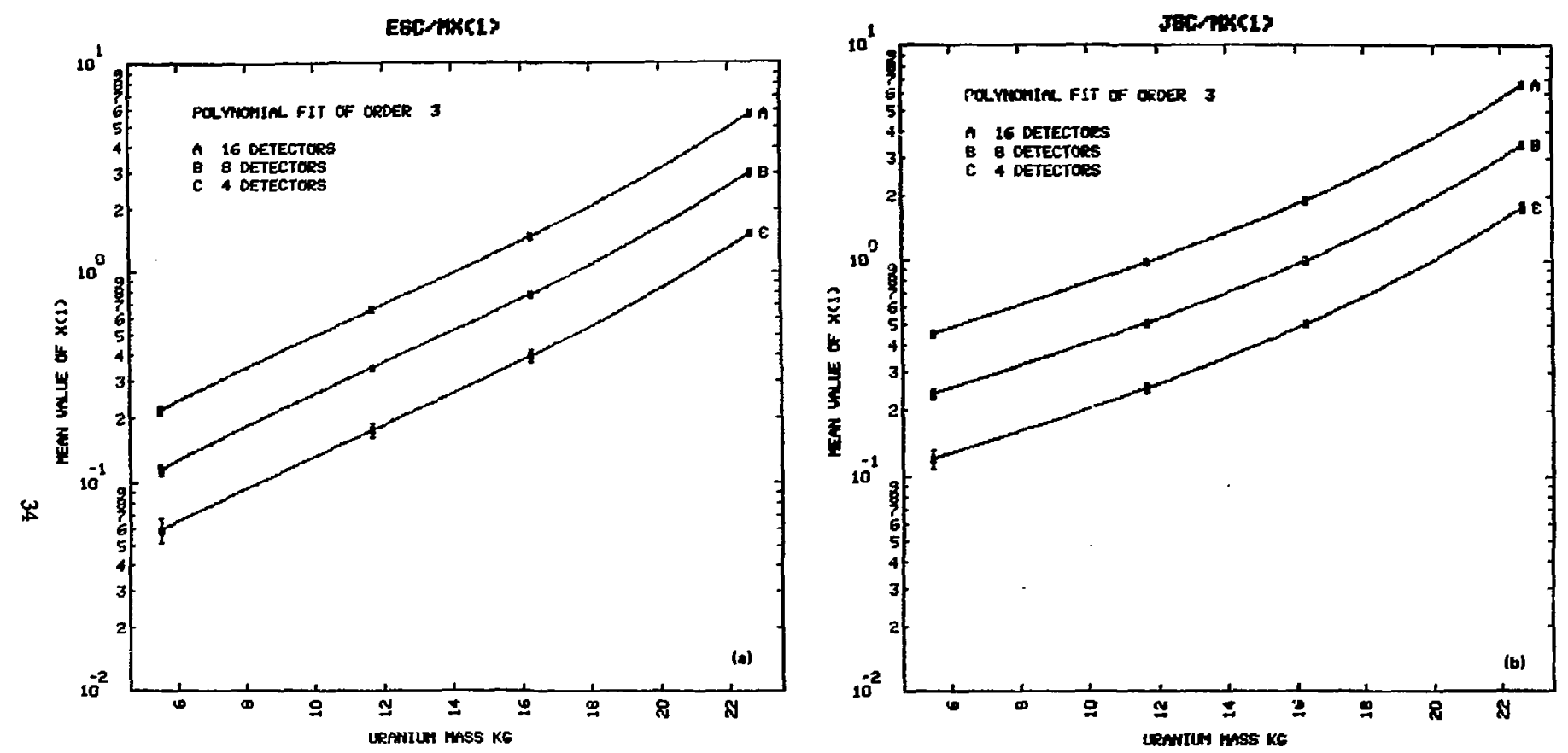

Fig. 13. Coefficient of the serial-covariance-to-mean ratio function plotted against the uranium mass in the assembly. Each datum is the mean of 20 determinations. Error bars are centered at the mean and are $2 \sigma$ long, where $\sigma$ is the sample standard deviation calculated from the 20 delerminations. A full disk of data was processed for each determination. (a) was driven by the nonfission (PuBe) source; (b) was driven by the fission $\left(^{252} \mathrm{Cf}\right.$ ) source. 

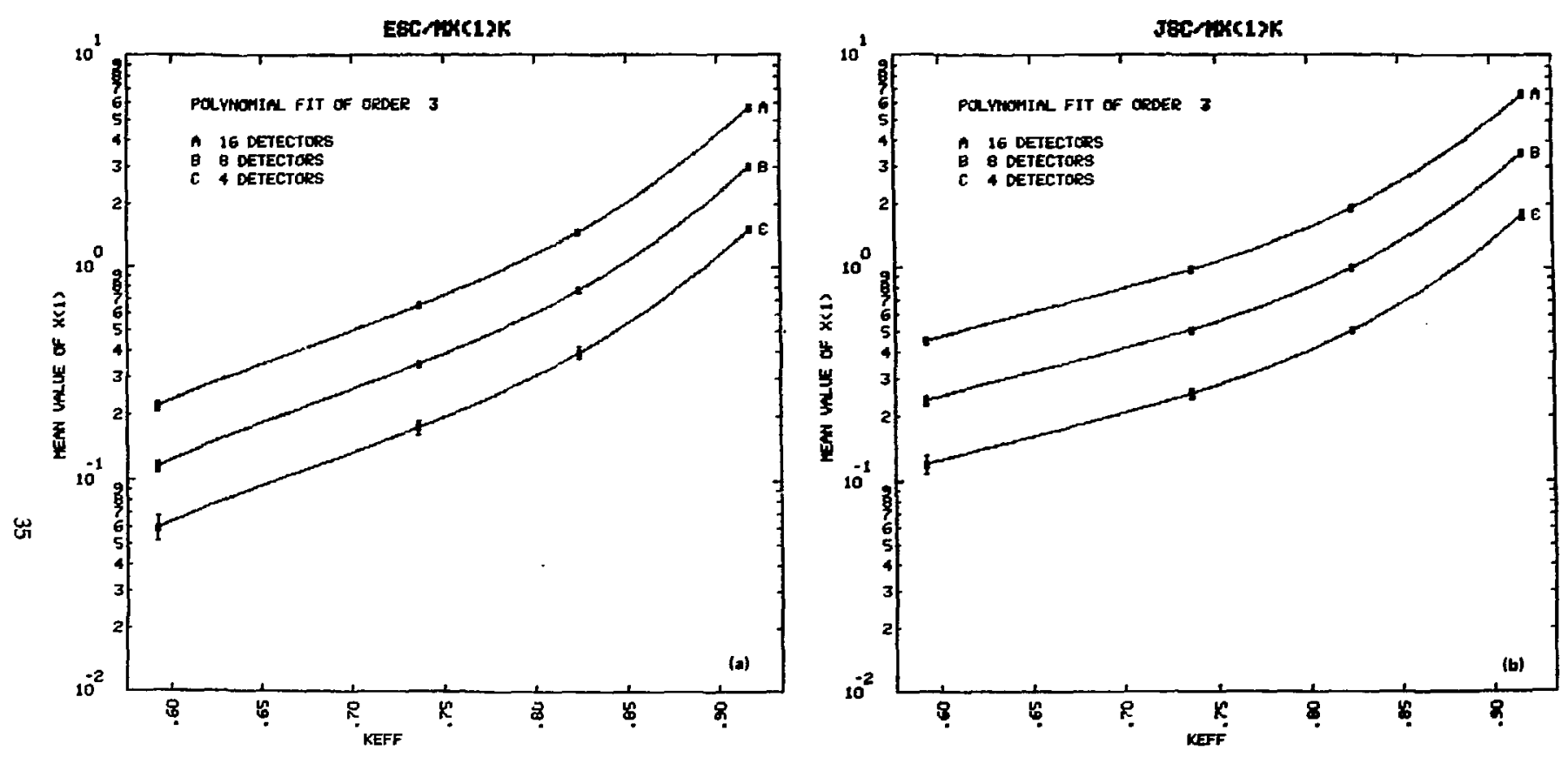

Fig. 14. Coefficient of the serial-covariance-to-mean ratio plotted against the calculated multiplication factor $(k)$ of the assembly. Each datum is the mean of 20 determinations. Error bars are centered at the mean and are $2 \sigma$ long, where $\sigma$ is the sample standard deviation calculated from the 20 determinations. A full disk of data was processed for each determination. (a) was driven by the nonfission (PuBe) source. (b) was driven by the fission $\left({ }^{252} \mathrm{Cf}\right)$ source. 

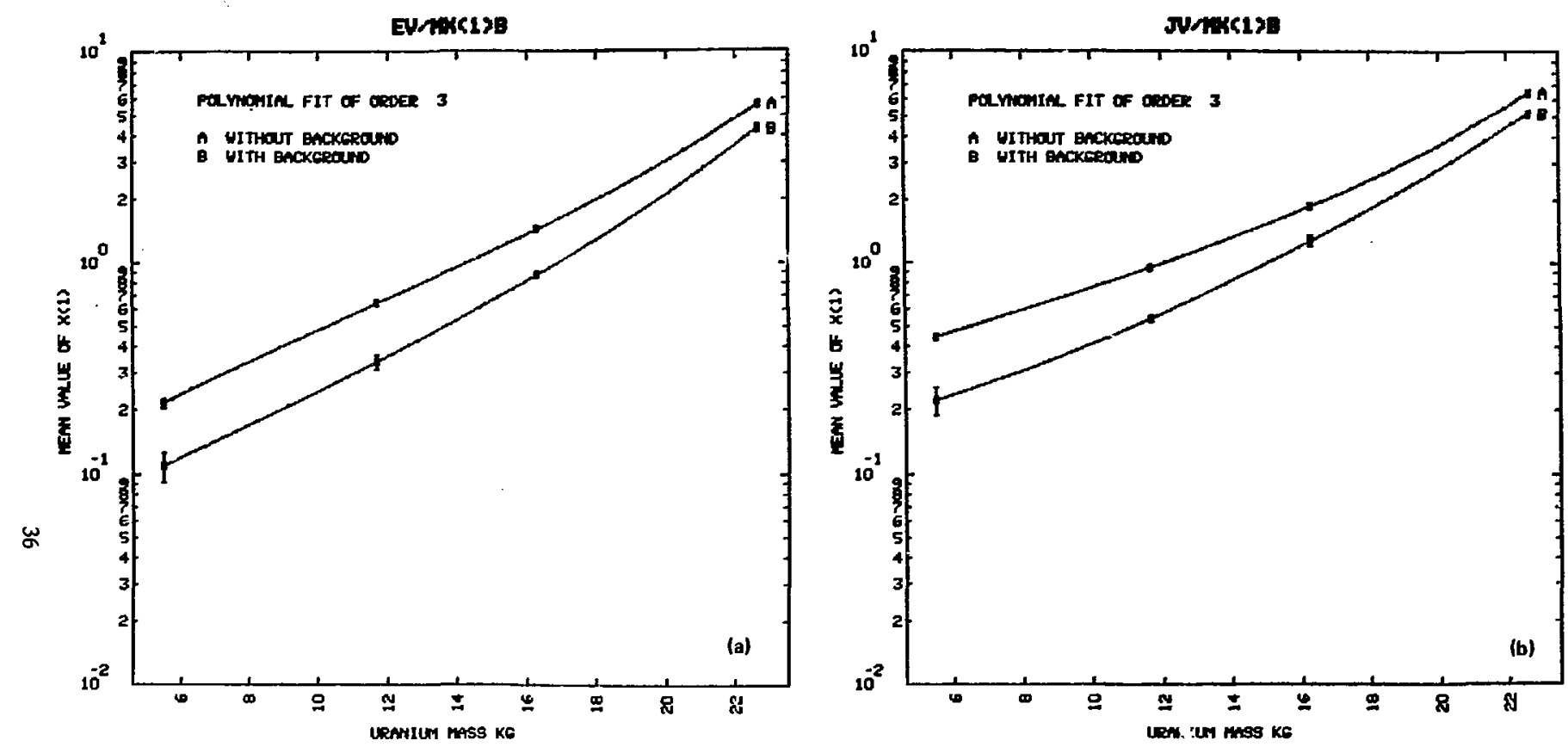

Fig. 15. Asymptotic increment in the variance-to-mean ratio plotted against the uranium mass in the assembly. Each datum of the upper curve is the mean of $\mathbf{2 0}$ deferminations; each datum of the lower curve is the mean of $\mathbf{5}$ determinations. The data of the lower curve were taken with an extraneous background count of approximately 2000 counts/second. Error bars are centered at the mean and are $2 \sigma$ long, where $\sigma$ is the standard deviation calculated from the 20 (5) determinations. A full disk of data was processed for each determination. All 16 detectors were active. (a) was driven by the non : $s$ sion (PuBe) source; (b) was driven by the fission $\left({ }^{252} \mathrm{Cf}\right.$ ) source. 

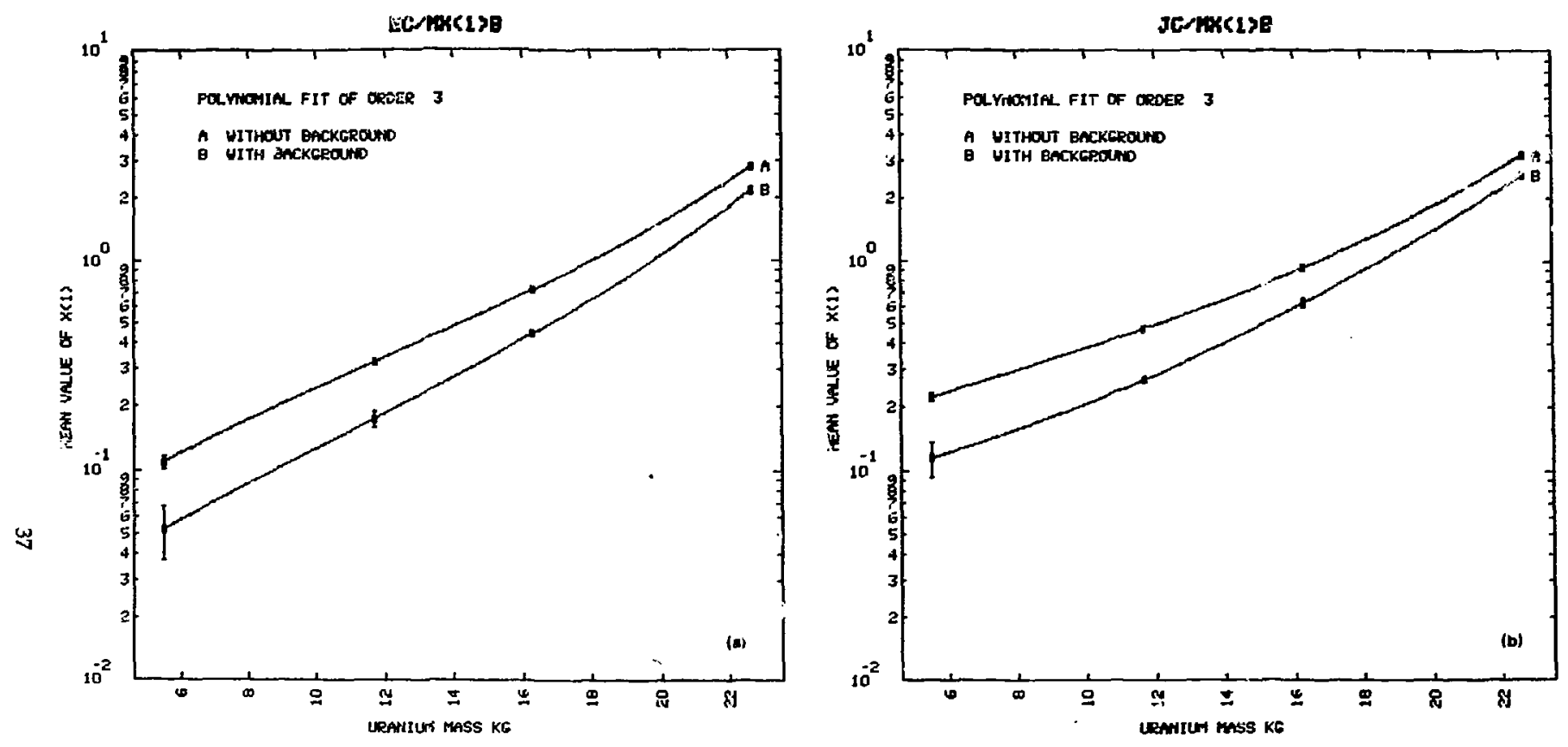

Fig. 16. Asymptotic value of the covariance-to-mean ratio plotted against the uranium mass in the assenbly. Each datum of the upper curve is the mean of 20 determinations; each datum of the lower curve is the mean of $t$ determinations. The data of the iower curve were takeit with an extraneous background count of approximately 2000 counts/second. Frror bars are centered at the mean and are $2 \sigma$ long, where $\sigma$ is the standard deviation calculated from the 20 (5) determinations. A full disk of data was processed for each determination. All 16 detectors were active. (aj was driven by the nonfission (PuBe) source; (b) was driven by the ission $\left({ }^{252} \mathrm{Cn}\right.$ ) source. 

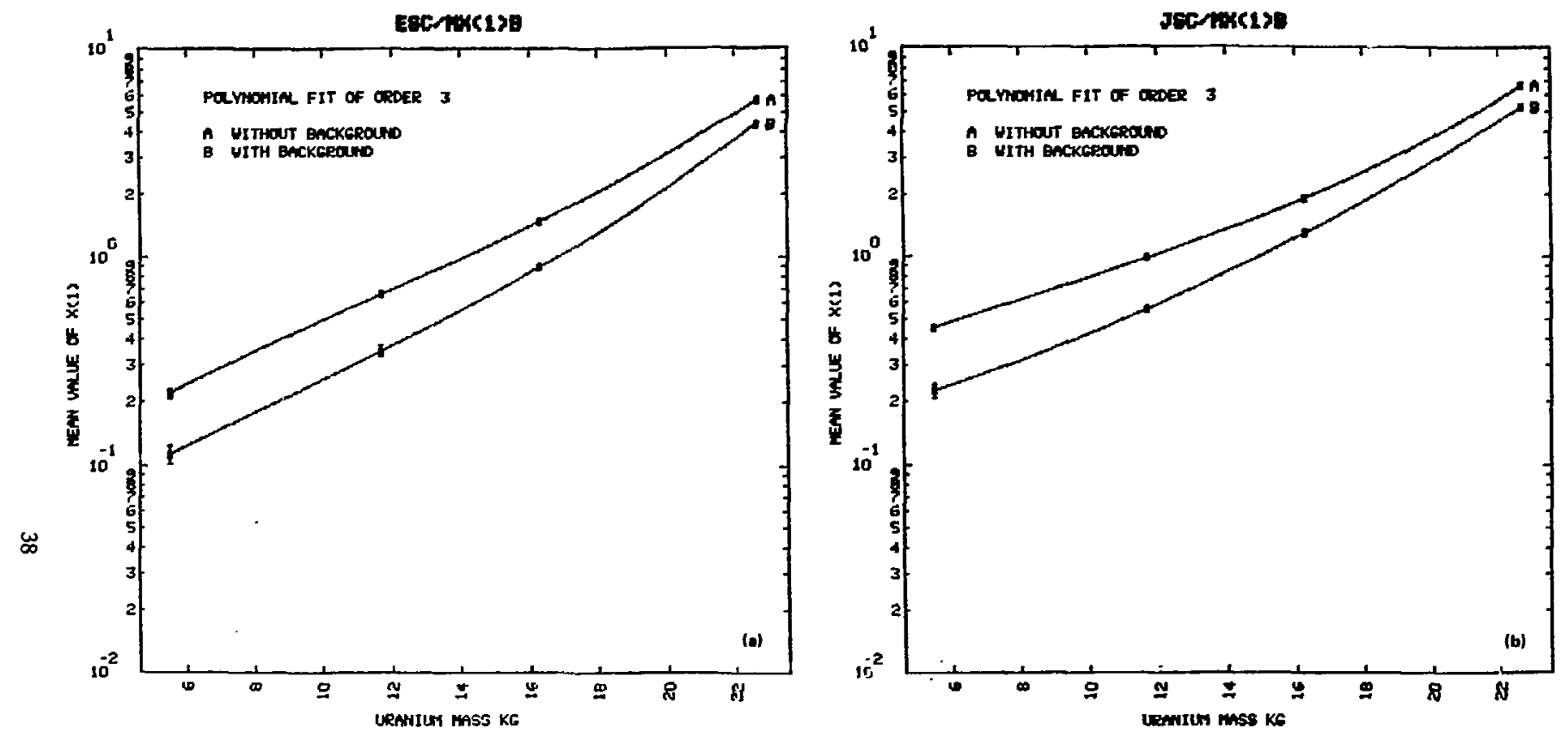

Fig. 17. Coefficient of the serial-covariance-to-rnean ratio function plotted against the uranium mass in the assembly. Each datum of the upper curve is the mean of 20 determinations; each datum of the lower curve is the mean of 5 determinations. The data of the lower curve were taken with an extraneous background count of approximately 2000 counts/second. Error bars are centered at the mean and are $2 \alpha$ long, where $\sigma$ is the standard deviation calculated from the 20 (5) determinations. A full disk of data was processed for each determination. All 16 detectors were active. (a) was driven by the nonfission ( $\mathrm{PuBe}$ ) source; (b) was driven by the fission $\left({ }^{252} \mathrm{Cf}\right)$ source. 
Observe that

$b=\frac{1}{1+r}$

where $r=$ (mean count rate from extraneous neutrons)/(mean count rate from assembly neutrons). Factor $b$ multiplies the asymptotic increment in the variance-to-mean ratio. It is 1 if no extraneous background exists $(r=0)$ and tends toward 0 as the extraneous background increases ( $r$ large).

Table 1 shows some effects of background on the asymptotic increment in the variance-tomean ratio discriminator. Compare the last two columns and see that the experimental and theoretical results agree reasonably well except perhaps in the first row. Entries in the first row show results obtained with one fuel shell while the assembly was driven with the nonfission source. That case delivers a weak signal, especially when the extraneous background source is present. 1 ascribe the discrepancy in the first row to that weakness.

\section{Mean Values of Estimators of Assembly Decay Constant}

As discussed above, least-squares fits to theoretical formulae were used to estimate values of the three discriminators. For each such estimate, an estimate of the assembly time constant was also obtained. The following three figures, Figs. 18, 19, and 20, summarize the set of estimators of $\tau$, the assembly time constant.

Observe from the figures that the time constant changes slowly with fuel mass over the range of masses available and that the estimators of time constant are noisy. Hence, estimators of assembly time constant were not useful as discriminators.

\section{Sample of Two-Parameter Least-Squares Fits}

\section{Variance-to-Mean Ratio}

Figure 21 shows five examples of least-squares fits to the theoretical variance-to-mean ratio functions. The five graphs show results for 0-, 5.5-, 11.7-, 16.3-, and 22.6-kg loadings with the assembly driven by the nonfission source. See the neighborhood of Eq. (48) in the chapter describing the probabilistic model for a discussion of the theoretical formula and the two parameters.

Table 1. Asymptotic increment in the variance-to-mean ratio with and without extraneous background. Data correspond to those used to generate Fig. 15. All detectors active.

\begin{tabular}{lcllcc}
\hline \multirow{2}{*}{$\begin{array}{c}\text { Source } \\
\text { type }\end{array}$} & $\begin{array}{c}\text { No. of } \\
\text { fuel } \\
\text { shells }\end{array}$ & WOB $^{6}$ & WB & WOB/WB & $b=1 /(1+r)^{\circ}$ \\
\hline Nonfission & 1 & 0.217 & 0.109 & 0.50 & 0.42 \\
& 2 & 0.640 & 0.338 & 0.53 & 0.53 \\
& 3 & 1.44 & 0.873 & 0.61 & 0.61 \\
& 4 & 5.59 & 4.30 & 0.77 & 0.75 \\
Fission & & & & & \\
& 1 & 0.444 & 0.222 & 0.50 & 0.49 \\
& 2 & 0.952 & 0.545 & 0.57 & 0.58 \\
& 3 & 1.87 & 1.28 & 0.68 & 0.67 \\
& 4 & 6.43 & 5.16 & 0.80 & 0.81 \\
\hline
\end{tabular}

Measured asymptotic increment in the variance-to-mean ratio.

'Without extraneous background; based on 20 disks of data.

'With extraneous background; based on 5 disks of data.

$d_{r}=$ (WB count rate - WOB count rate)/(WOB count rate). The numerator of $r$ varied somewhat with different runs but was nominally 2000 counts/second. 

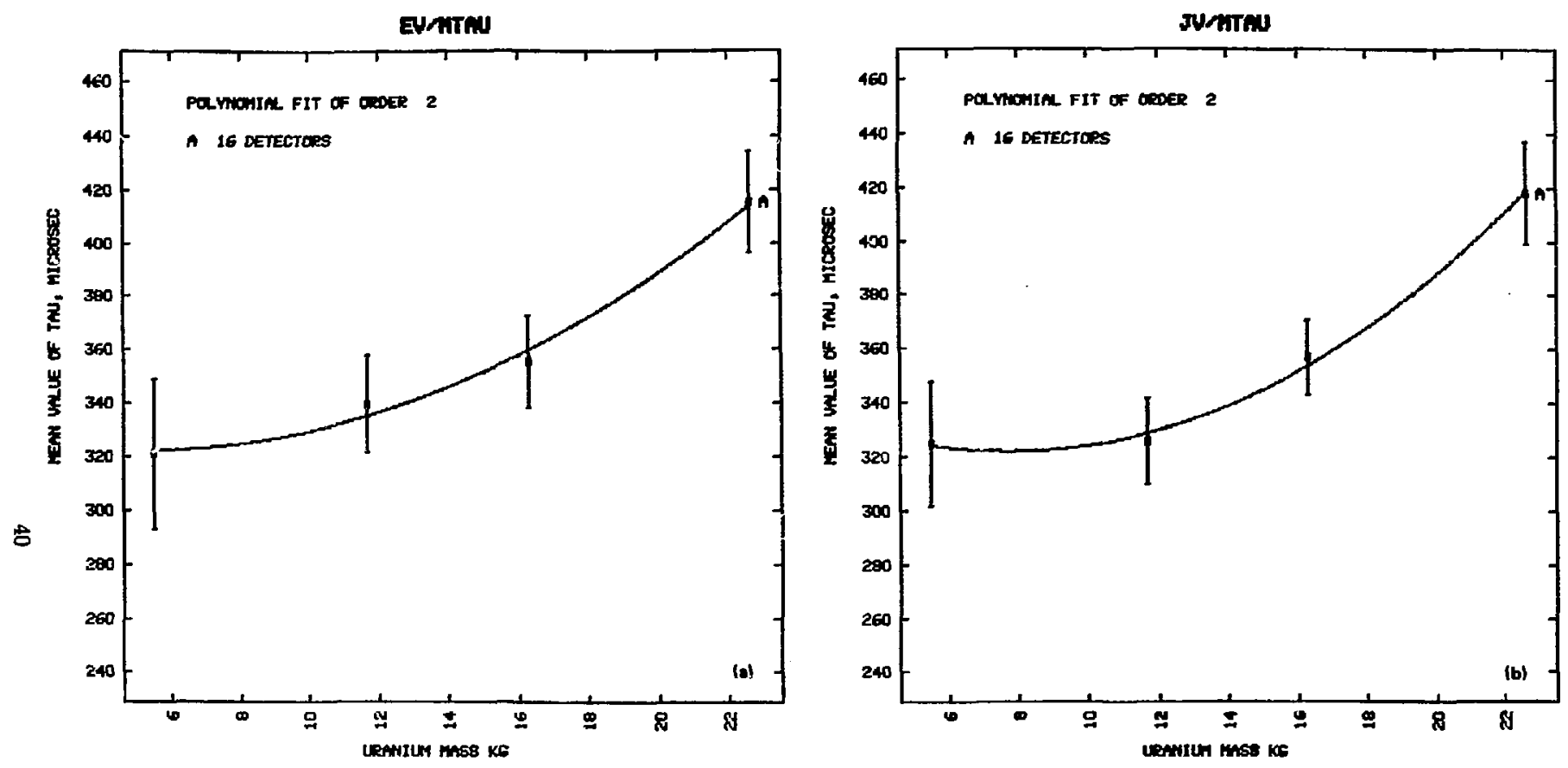

Fig. 18. Assembly time decay constant plotted against the uranium mass in the assembly. Each datum is the mean of 20 determinations from leastsquares fits to the variance-to-mean ratio function. Error bars are centered at the sample mean and are $2 \sigma$ long, where $\sigma$ is the sample standard deviation calculated from the 20 determinations. A full disk of data was processed for each determination. All 16 detectors were active. (a) was driven by the nonfission (PuBe) source; (b) was driven by the fission $\left(^{252} \mathrm{Cf}\right.$ ) source. 

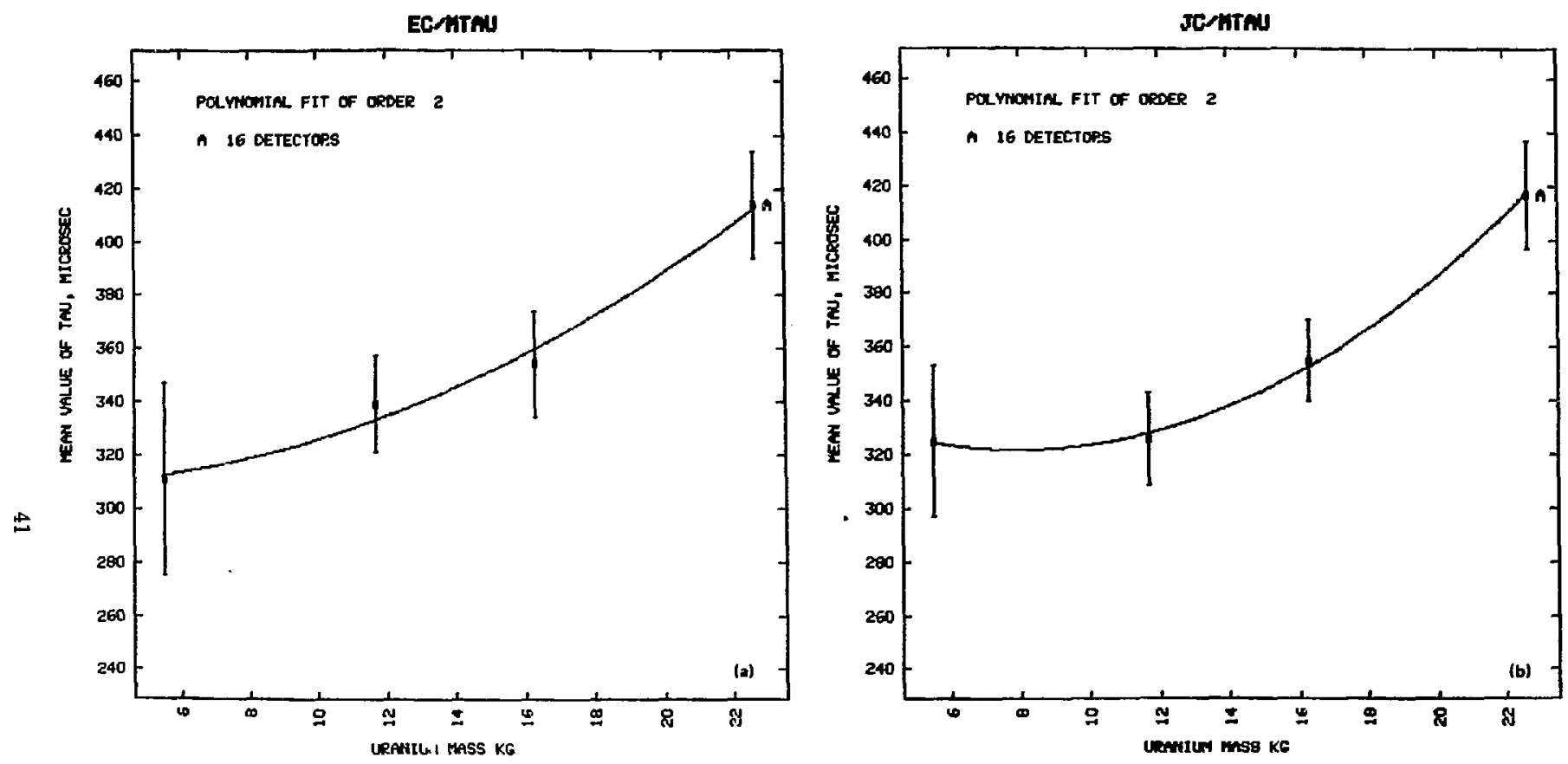

Fig. 19. Assembiy time decay constant plotted against the uranium mass in the assembly. Each datum is the mean of 20 determinations from leastsquares fits to the covariance-to-mean ratio function. Error bars are centered at the sample mean and are $2 \sigma$ long, where $\sigma$ is the sample standard deviation calculated from the 20 determinations. A full disk of data was processed for each determination. All 16 detectors were active. (a) was driven by the nonfission (PuBe) source; (b) was driven by the fission $\left({ }^{252} \mathrm{Cf}\right)$ source. 

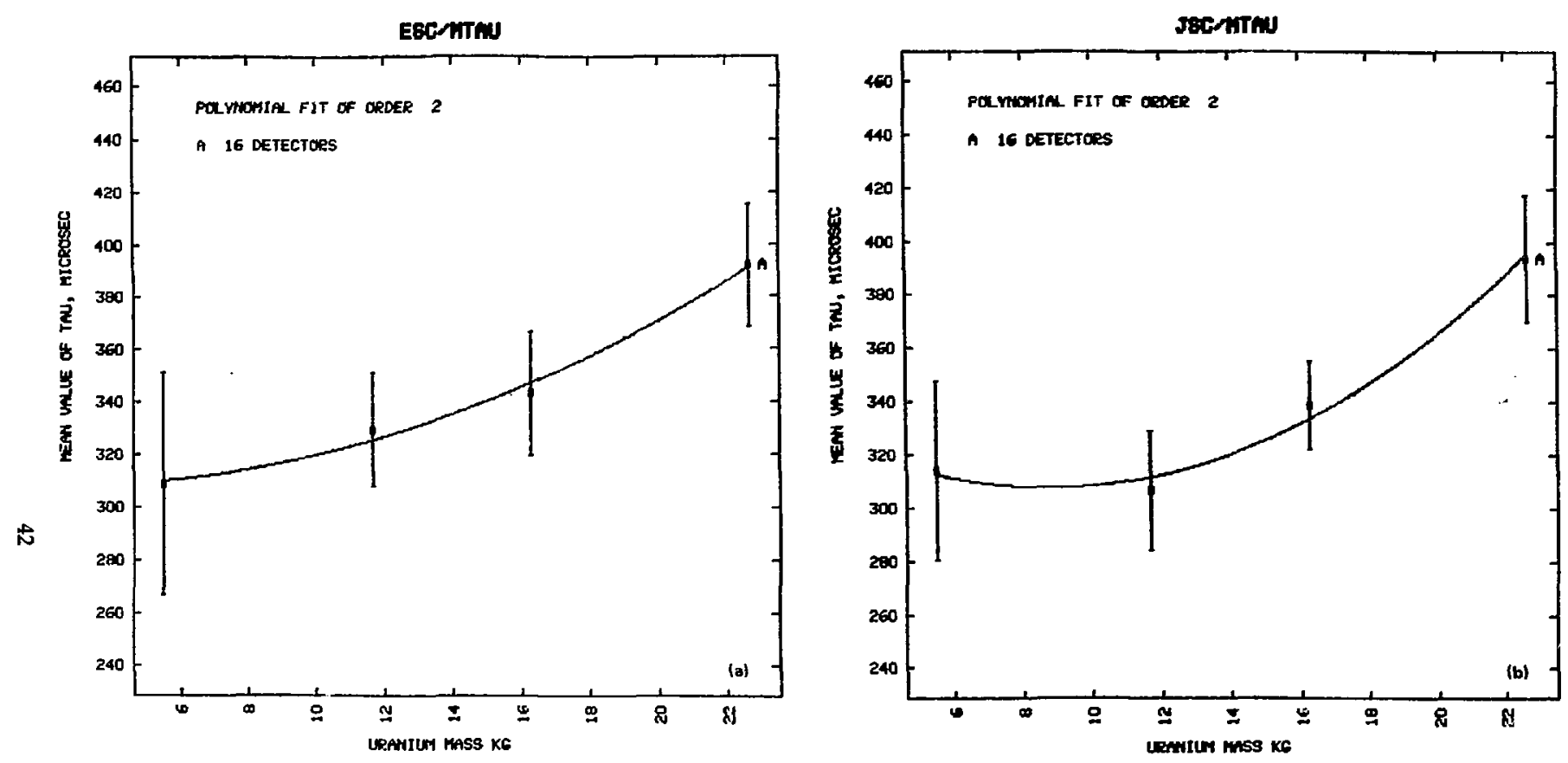

Fig. 20. Assembly time decay constant plotted against the uranium mass in the assembly. Each datum is the mean of 20 determinations from leastsquares fits to the serial-covariance-to-mean ratio function. Error bars are centered at the sample mean and are $2 \sigma$ long, where $\sigma$ is the sample standard deviation calculated from the 20 determinations. A full disk of data was pıocessed for each determination. All 16 detectors were active. (a) was driven by the nonfission (PuBe) source; (b) was driven by the fission $\left(^{252} \mathrm{Cf}\right.$ ) source. 

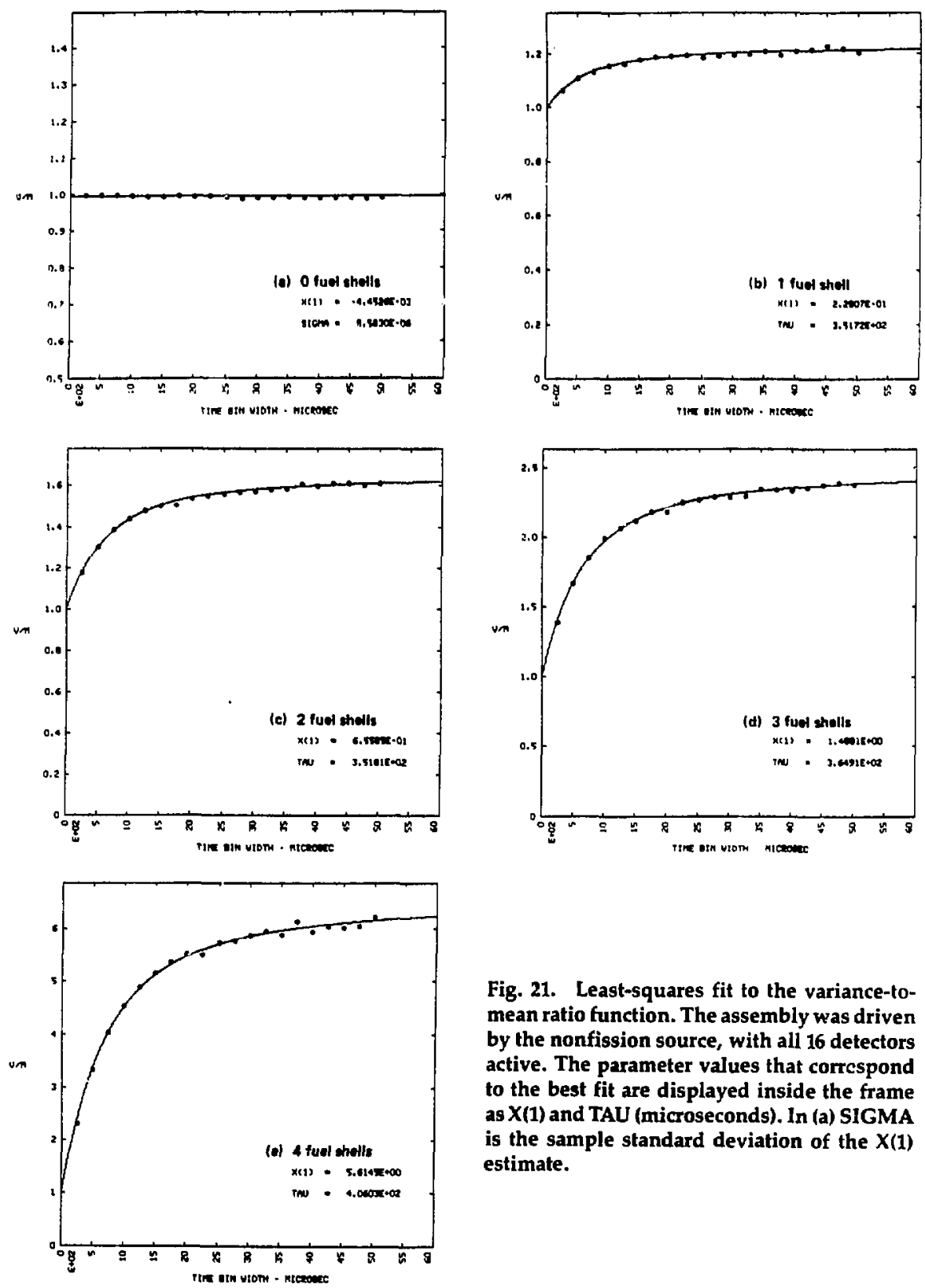

Fig. 21. Least-squares fit to the variance-tomean ratio function. The assembly was driven by the nonfission source, with all 16 detectors active. The parameter values that corrcspond to the best fit are displayed inside the frame as X(1) and TAU (microseconds). In (a) SIGMA is the sample standard deviation of the $X(1)$ estimate. 
Here is a description of how the points on the graphs were obtained. We have a record of detections spread out along a segment, $[0, T]$, say, of the time line. Choose a time interval, $\Delta t$, and let $n$ equal the integer part of $T / \Delta t$. Divide the segment of length $T$ into $n$ segments of length $\Delta t$ and discard the remainder bin of length less than $\Delta t$. Now, for $1 \leq i \leq n$, let $X_{i}(\Delta t)=$ the number of detections in bin $i$. Then

$$
V / M(\Delta t)=\frac{\sum_{i=1}^{n} x_{i}^{2}(\Delta t)}{\sum_{i=1}^{n} x_{i}(\Delta t)}-\frac{1}{n} \sum_{i=1}^{n} x_{i}(\Delta t)
$$

is the estimator I used to generate the ordinates of the left-most points of the graphs in Fig. 21. The subsequent points were generated by incrementing the time bin width in steps of length $\Delta t$, and making obvious changes in Eq. (71).

Figure 22 is Fig. 21 repeated except that all five curves are plotted on a common frame to afford easy comparison.

Figures 23 and 24 are similar to Figs. 21 and 22 except that rigs. 23 and 24 show results obtained when the multiplying assembly was driven by the californium- 252 fission source. Observe that even with no fuel ( 0 -shells case) the asymptotic variance-to-mean ratio is slightly greater than 1 because of the randomness in the number of neutrons emitted in the spontaneous fission of a californium-252 nucleus.

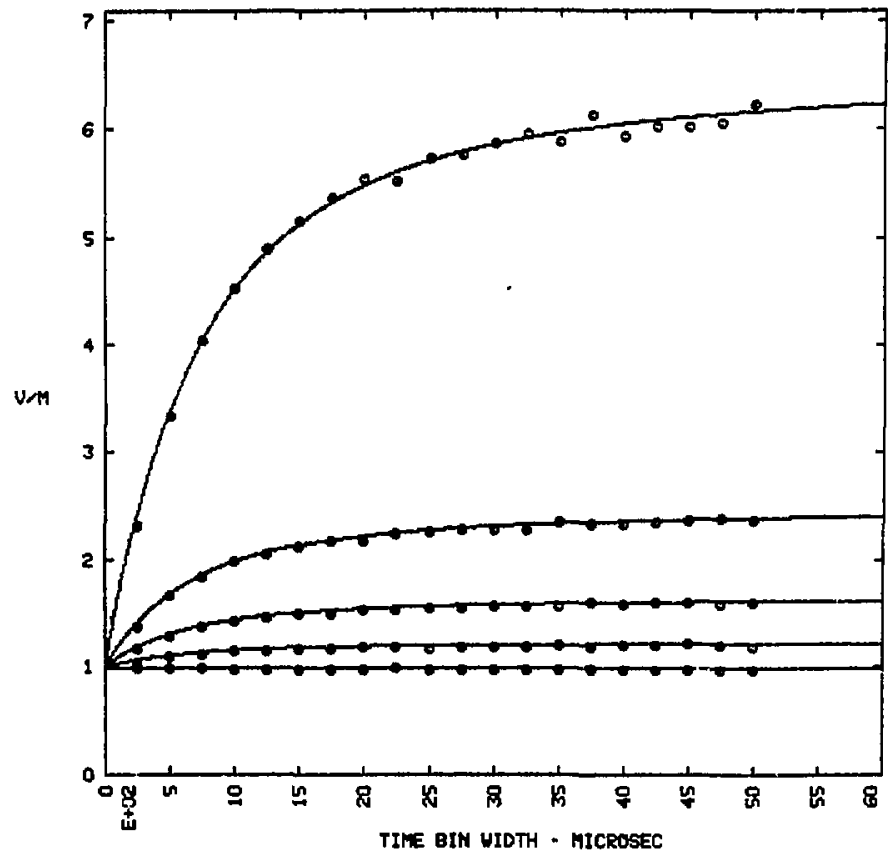

Fig. 22. Least-squares fit to the variance-to-mean ratio function. The data displayed here are identical to those displayed in Fig. 21; this format was chosen to afford easy visual comparison of the effects of different fuel loadings. 

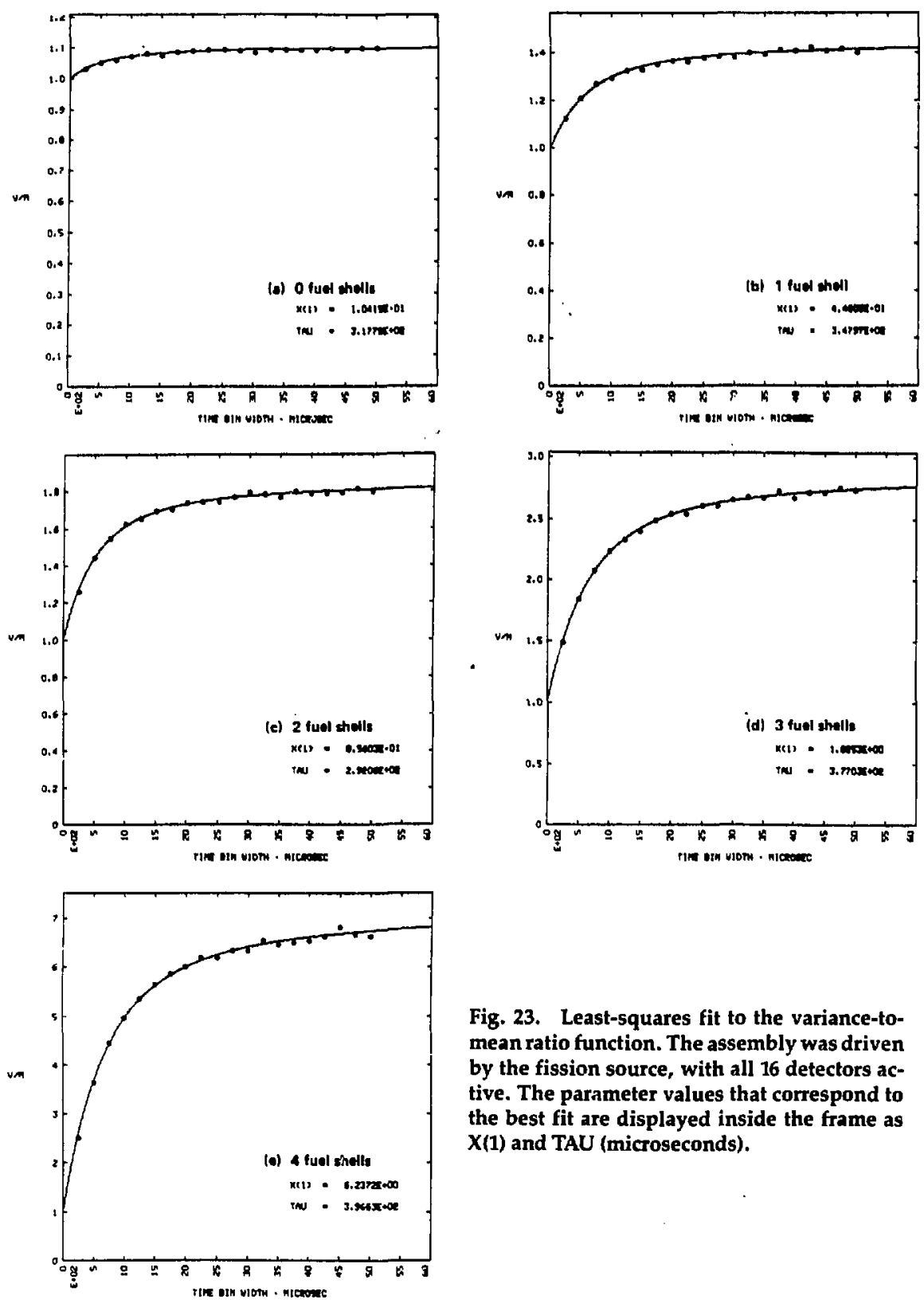

Fig. 23. Least-squares fit to the variance-tomean ratio function. The assembly was driven by the fission source, with all 16 detectors active. The parameter values that correspond to the best fit are displayed inside the frame as $X(1)$ and TAU (microseconds). 


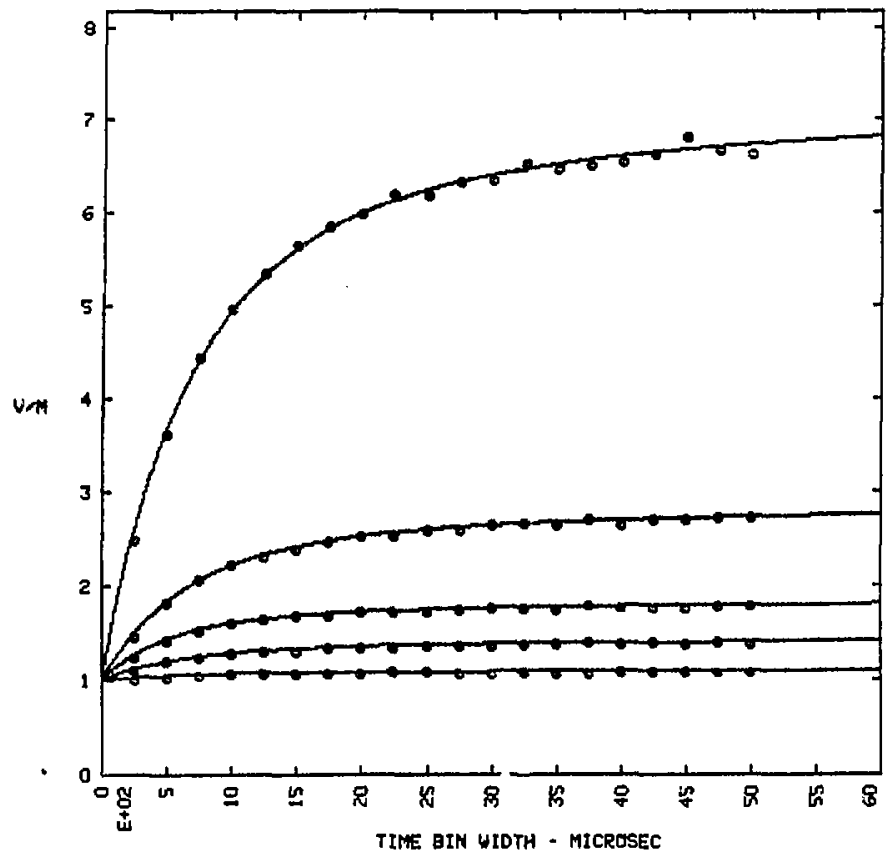

Fig. 24. Least-squares fit to the variance-to-mean ratio function. The data displayed here are identical to those displayed in Fig. 23; this fo-mat was chosen to afford easy visual comparison of the effects of different fuel loadings.

\section{Covariance-to-Mean Ratio}

Figures 25 through 28 show examples of least-squares-fits to the theoretical covariance-tomean ratio function. Figures 25 and 26 display identical data but with different ordinate scales. Similarly, Figs. 27 and 28 display identical data. See the neighborhood of Eq. (52) in the chapter describing the probabilistic model for a discussion of the theoretical covariance-to-mean ratio formula and the two parameters chosen by the least-squares-fit procedure.

Except for details I now discuss, the data points in Figs. 25 through 28 were obtained by a procedure similar to that described above for the variance-to-mean ratio case.

Here are those details. Out of the 16 available detectors, I picked two subsets. For Figs. 25 through 28 set \#1 consisted of the odd-numbered detectors and set \#2 consisted of the evennumbered detectors. For $1 \leq i \leq n$, let $X_{i}(\Delta t)=$ the number of detections recorded by detector set "1 in the ith time bin. Define $Y_{i}(\Delta t)$ similarly for detector set $" 2$. Then

$$
C / M(\Delta t)=\frac{\sum_{i=1}^{n} x_{i}(\Delta t) Y_{i}(\Delta t)}{\left(\sum_{i=1}^{n} x_{i}(\Delta t)\right)^{1 / 2}\left(\sum_{i=1}^{n} \gamma_{i}(\Delta t)\right)^{1 / 2}}-\frac{1}{n}\left(\sum_{i=1}^{n} x_{i}(\Delta t)\right)^{1 / 2}\left(\sum_{i=1}^{n} \gamma_{i}(\Delta t)\right)^{1 / 2}
$$

is the estimator I used to generate the left-most points on the graphs in Figs. 25 through 28 . The remaining points were generated by incrementing the time bin width as described for the variance-to-mean ratio case. 

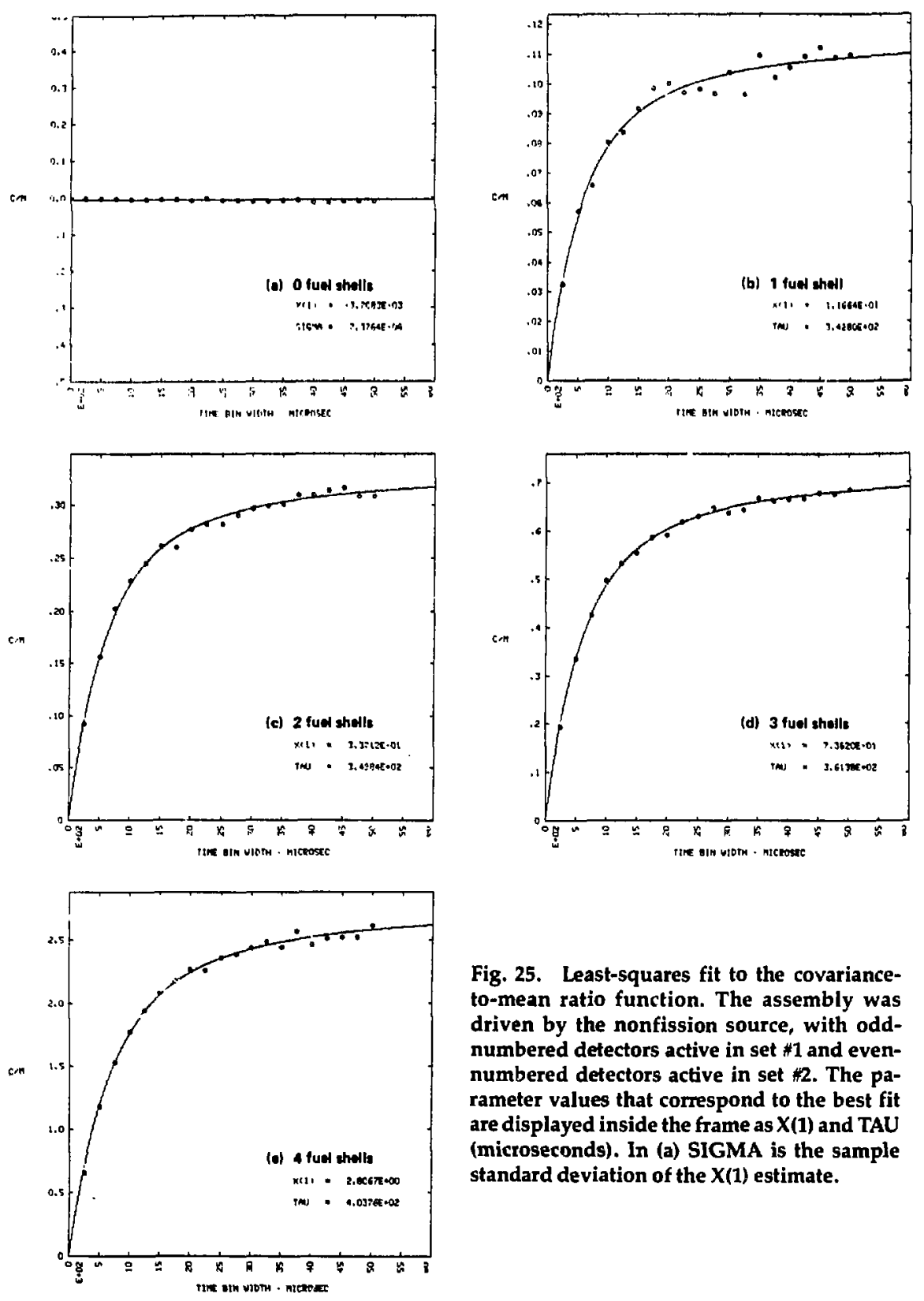

Fig. 25. Least-squares fit to the covarianceto-mean ratio function. The assembly was driven by the nonfission source, with oddnumbered detectors active in set $\# 1$ and evennumbered detectors active in set $\$ 2$. The parameter values that correspond to the best fit are displayed inside the frame as $X(1)$ and TAU (microseconds). In (a) SIGMA is the sample standard deviation of the $X(1)$ estimate. 


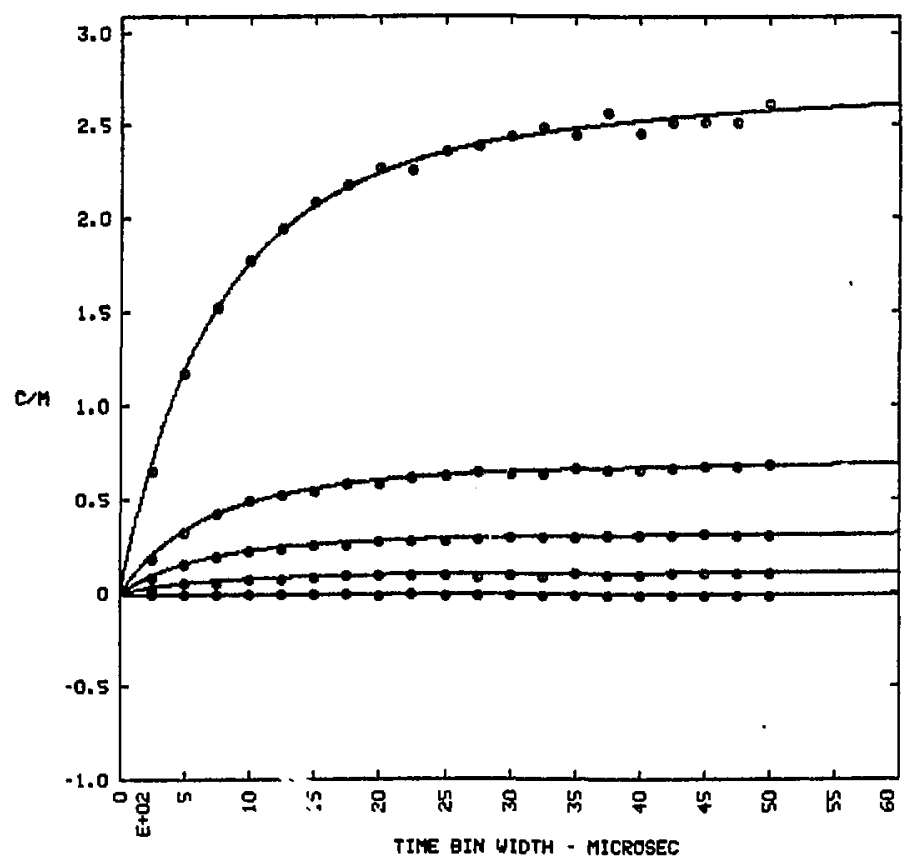

Fig. 26. Least-squares fit to the covariance-to-mean ratio function. The data displayed here are identical to those displayed in Fig. 25; this format was chosen to afford easy visual comparison of the effects of different fuel loadings. 

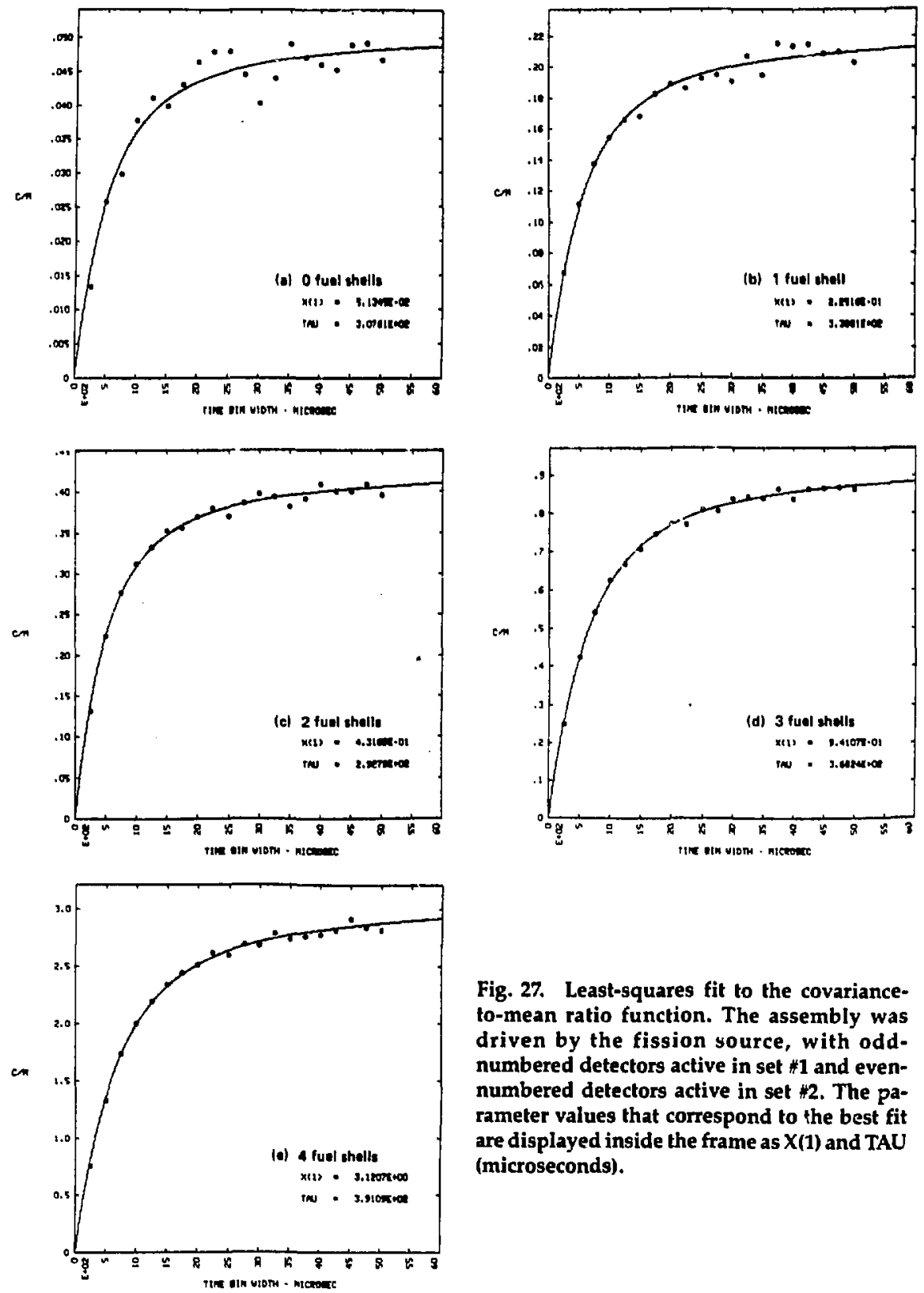

Fig. 27. Least-squares fit to the covarianceto-mean ratio function. The assembly was driven by the fission source, with oddnumbered detectors active in set $\# 1$ and evennumbered detectors active in set $\$ 2$. The parameter values that correspond to the best fit are displayed inside the frame as $X(1)$ and TAU (microseconds). 


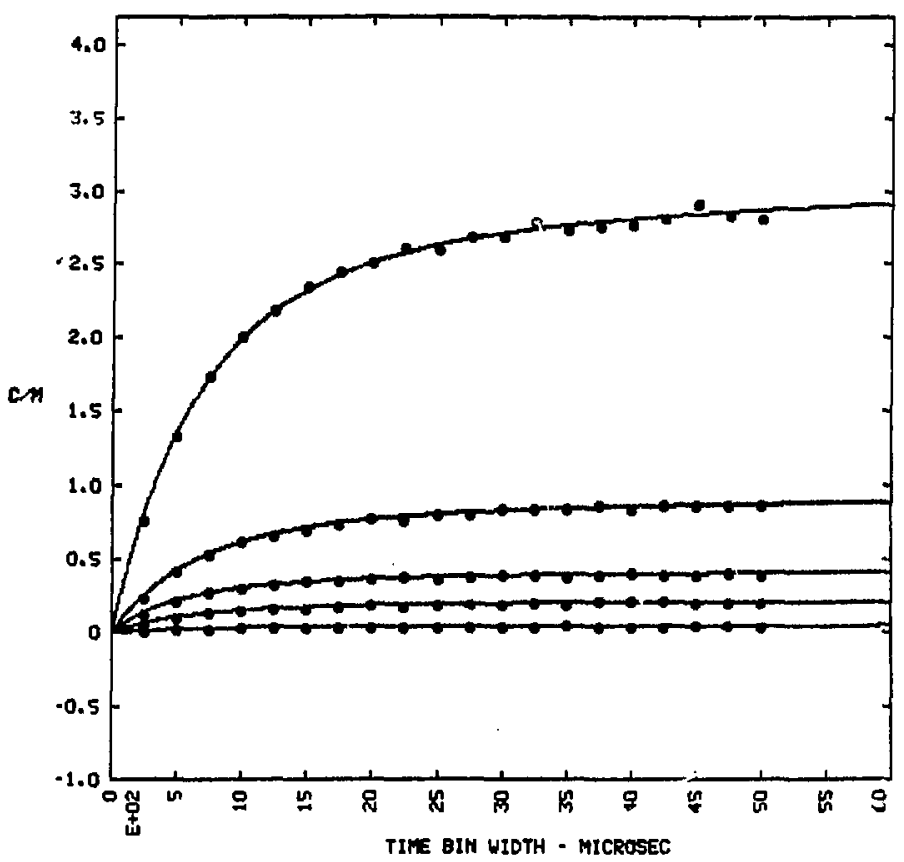

Fig. 28. Least-squares fit to the covariance-to-mean ratio function. The data displayed here are identical to those displayed in Fig. 27; this format was chosen to afford easy visual comparison of the effects of different fuel loadings.

\section{Serial-Covariance-to-Mean Ratio}

Figures 29 through 32 display examples of least-squares-fits to the theoretical serialcovariance-to-mean ratio function. Figures 29 and 30 display identical data but with different ordinate scales. Similarly, Figs. 31 and 32 show identical data. See the neighborhood of Eq. (62) in the chapter describing the probabilistic model for a discussion of the theoretical serial-covariance-tomean rat: $\rightarrow$ formula and the two parameters chosen by the least-squares-fit procedure.

Except for details that follow immediately below, the data points in Figs. 29 through 32 were obtained by a procedure similar to that outlined above for the variance-to-mean ratio case.

Here are the details that are specific to the serial-covariance-to-mean ratio case. For $1 \leq i \leq n$, let $X_{i}(\Delta I)=$ the number of detections in bin $i$ of widit $\Delta t$. Then

$$
\operatorname{SC/M}(\Delta t)=\frac{n}{n-1} \frac{\sum_{i=1}^{n-1} x_{i}(\Delta t) X_{i+1}(\Delta t)}{\sum_{i=1}^{n} x_{i}(\Delta t)}-\frac{1}{n} \sum_{i=1}^{n} x_{i}(\Delta t)
$$

is the estimator I used to generate the left-most points on the graphs in Figs. 29 through 32 . The remaining points were generated by incrementing the time bin width as described for the variance-to-mean ratio case. 

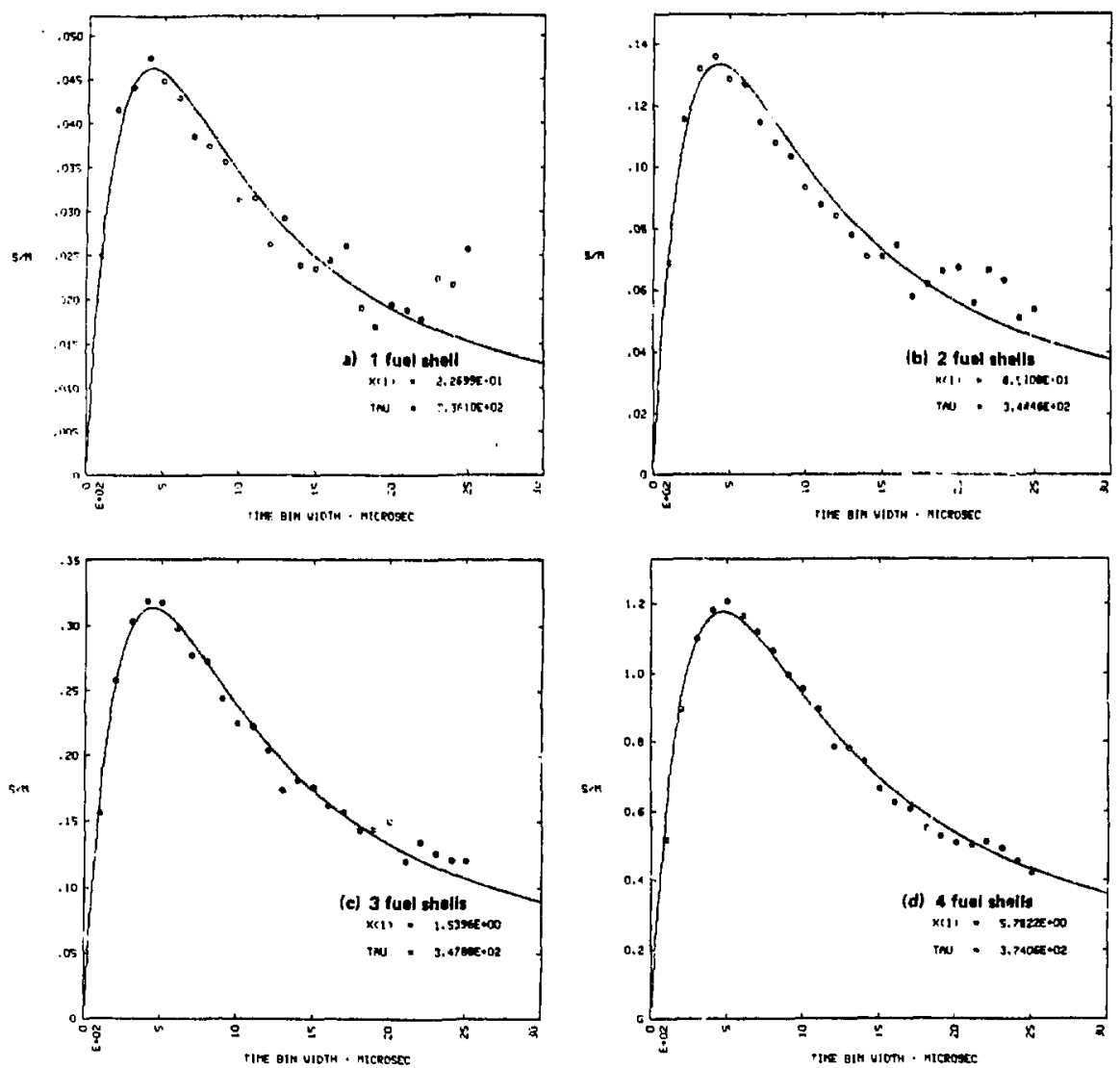

Fig. 29. Least-squares tit to the serial-covariance-to-mean ratio function. The assembly was driven by the nonfission source, with all detectors active. The parameter values that correspond to the best fit are displayed inside the frame as $X(1)$ and TAU (microseconds). 


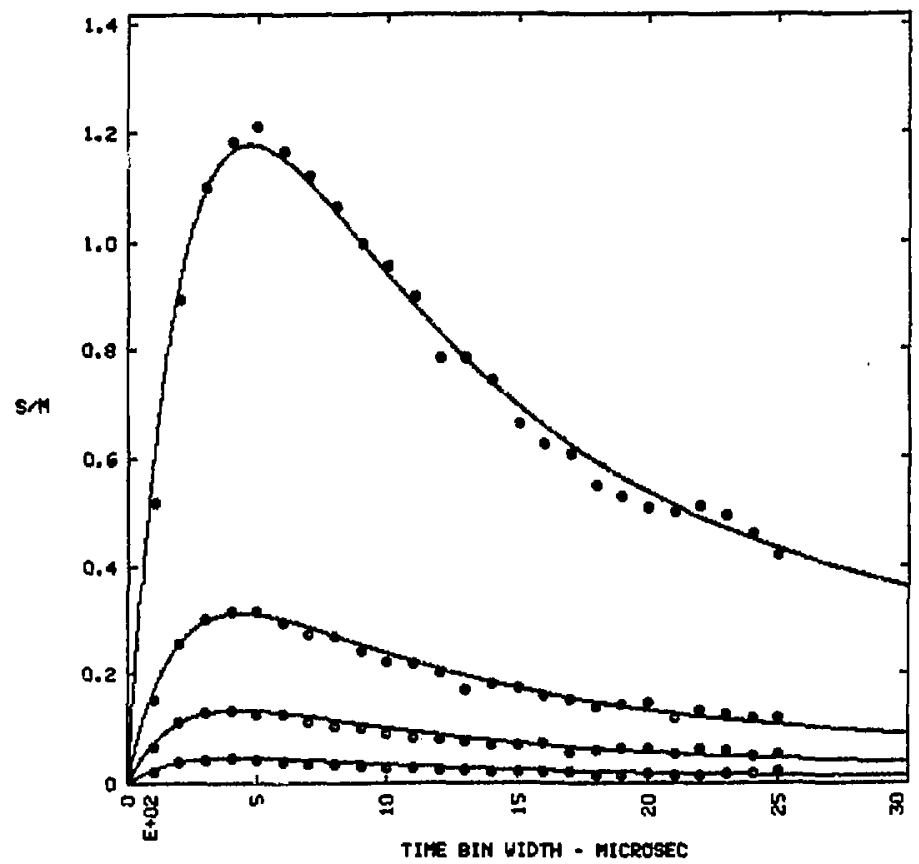

Fig. 30. Least-squares fit to the serial-covariance-to-mean ratio function. The data displayed here are identical to those displayed in Fig. 29; this format was chosen to afford easy visual comparison of the effects of different fuel loadings. 

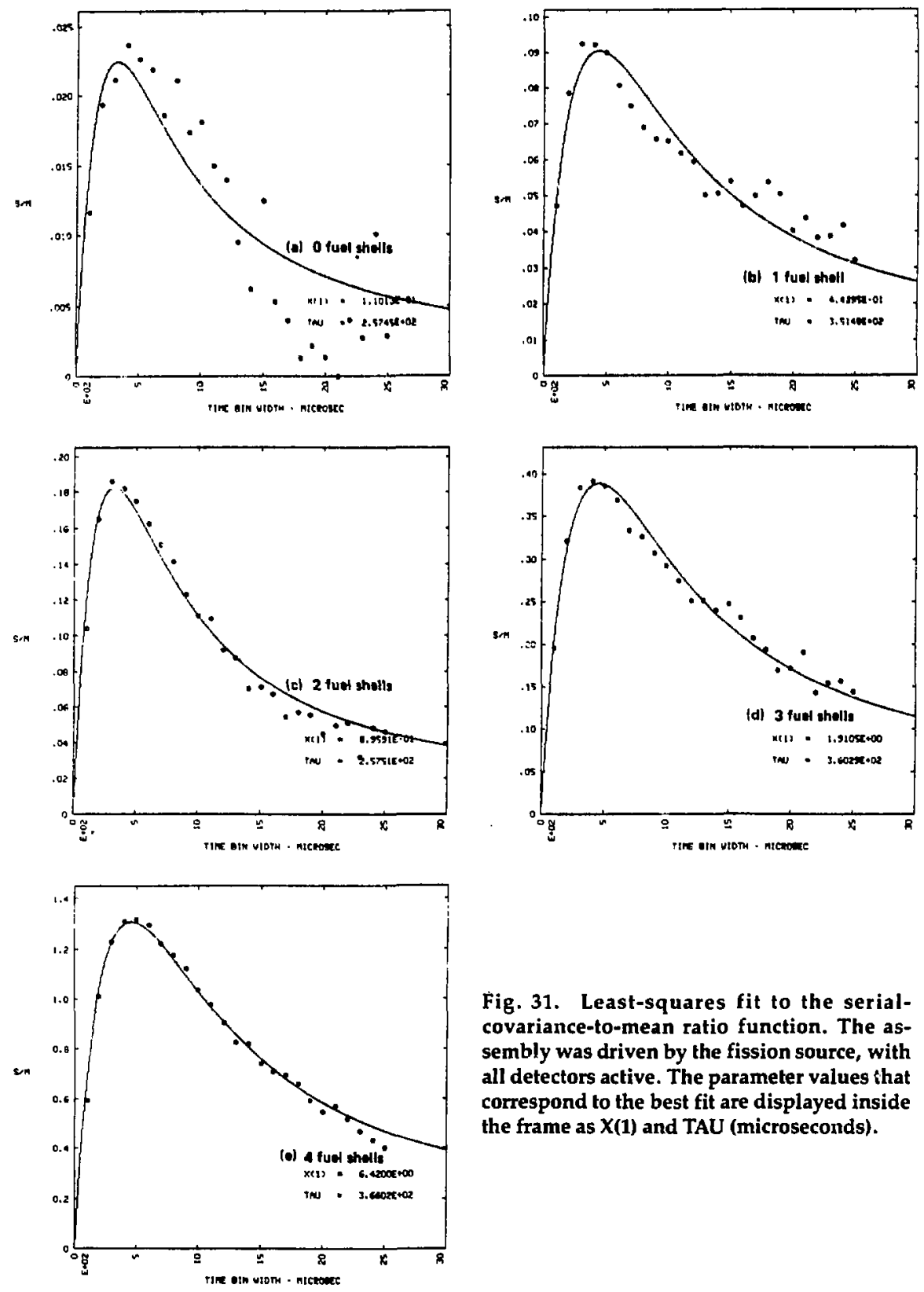

Fig. 31. Least-squares fit to the serialcovariance-to-mean ratio function. The assembly was driven by the fission source, with all detectors active. The parameter values that correspond to the best fit are displayed inside the frame as X(1) and TAU (microseconds). 


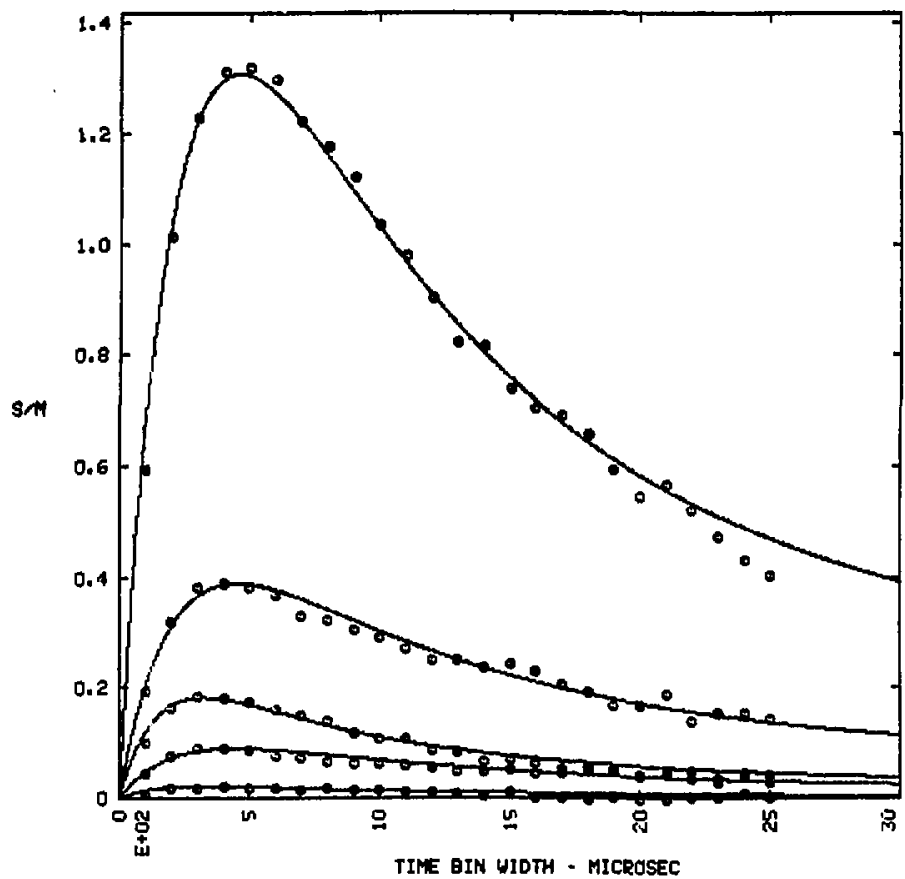

Fig. 32. Least-squares fit to the serial-covariance-to-mean ratio function. The data displayed here are identical to those displayed in Fig. 31; this format was chosen to afford easy visual comparison of the effects of different fuel loadings. 


\section{Assessment of Discriminator Performance}

We are at an appropriate point to start evaluating the relative performance of the three discriminators: variance-to-mean, covariance-to-mean, and serial-covariance-to-mean. To do so, I need some measure of goodness for a discriminator. I chose what I call decision rule performance characteristic (DRPC) for that measure. Apparently, communication and radar engineers were the first to use DRPCs to characterize detection algorithms, calling them receiver operating characteristics.

In describing a DRPC, it is convenient to strip away the details of the situation at hand and imagine a general setting. Consider a general binary decision problem in which two possible signals-strong and weak-are received by an observer. Either because of noise or because of the inherent statistical nature of the signals (our case is plagued with both), discrimination between a strong and a weak signal is not a trivial, deterministic task. Instead, it is necessary to construct a decision procedure that will, with acceptably high probability, discriminate between the two sig. nals.

I model the set-up by assuming that the received signal is a sample of a stochastic process. The two signal generators (strong and weak) emit sample paths of stochastic processes. I operate on a received sample path with some kind of processor that delivers a single number, $X$. If $X$ is greater than some chosen threshold, $x$, $[$ announce that the received signal is strong; otherwise I announce that the signal is weak. Observe that since each signal generator sends sample paths from a stochastic process, $X$ is a random variable. Hence, the decision procedure will sometimes yield false decisions.

Let $T$ be the "truth" random variable. If the received signal was sent by the strong generator, $T$ assumes the value 1 ; if by the weak generator, the value 0 . Let $D_{x}$ be the "decision" random variable if the threshold is set at $x$. If the decision procedure says "strong, " $D_{x}=1$; otherwise, $D_{x}=0$. Observe that $D_{x}$ is a member of a family of random variables indexed by the threshold setting. $X, T$, and $D_{x}$ are jointly distributed.

Now, of the various conditional probabilities that can be constructed, I attach names to two:

$P\left[D_{x}=1 \mid T=1\right] \quad$ (true high decision probability when the threshold is set at $x$ )

$\left.P\left|D_{Y}=1\right| T=0\right]$ (false high decision probability when the threshold is set at $x$ ).

Consider the map

$x \rightarrow\left(P\left[D_{x}=1 \mid T=0\right], P\left|D_{y}=1\right| T=1 \mid\right)$.

As the threshold $x$ rurs over its domain, its image traces a curve in the unit square. That curve is the DRPC. Observe that

$$
\begin{aligned}
& P\left[D_{x}=1 \mid T=1\right]=P[X>x \mid T=1], \\
& P\left[D_{x}=1 \mid T=0\right\}=P[X>x \mid T=0] .
\end{aligned}
$$

Decision rule performance characteristics tend to look something like the curves sketched in Fig. 33. For low values of the threshold $x$, the DRPC is located at the point $(1,1)$; tor high values at $(0,0)$. A good decision rule is represented by a DRPC that is everywhere near the union of the west and north boundaries of the unit square.

We return now to the situation at hand. Figures 34 through 45 show DRPCs for 12 different conditions, conditions created by variations in driving source (fission or nonfission), fuel mass (low or high), and number of detectors active $(16,8,4)$. 


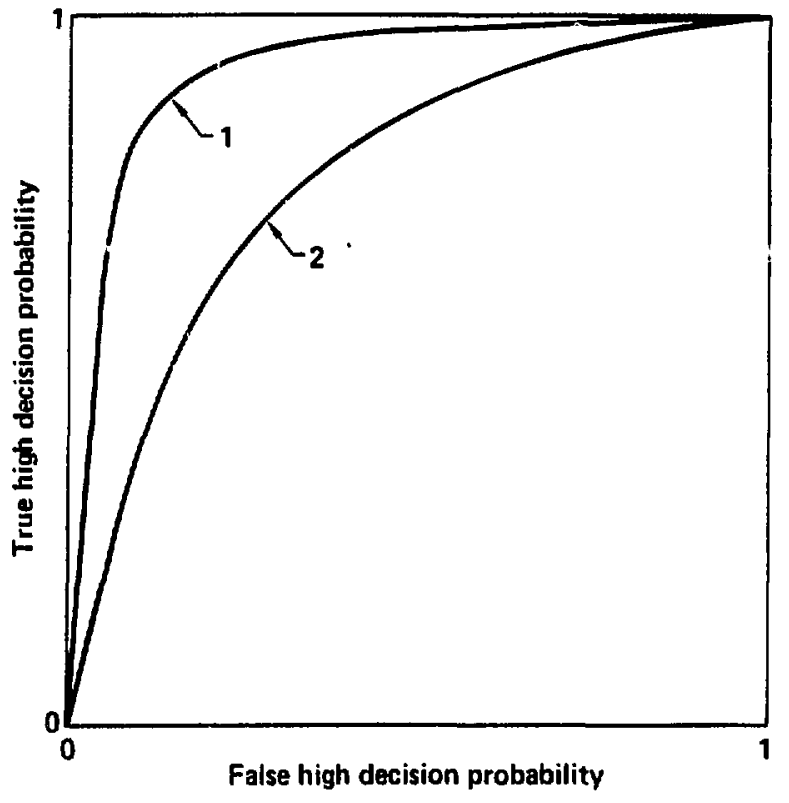

Fig. 33. Schematic DRPCs. Rule 1, by virtue of generating a curve closer to the north and west boundaries, is superior to Rule 2. 

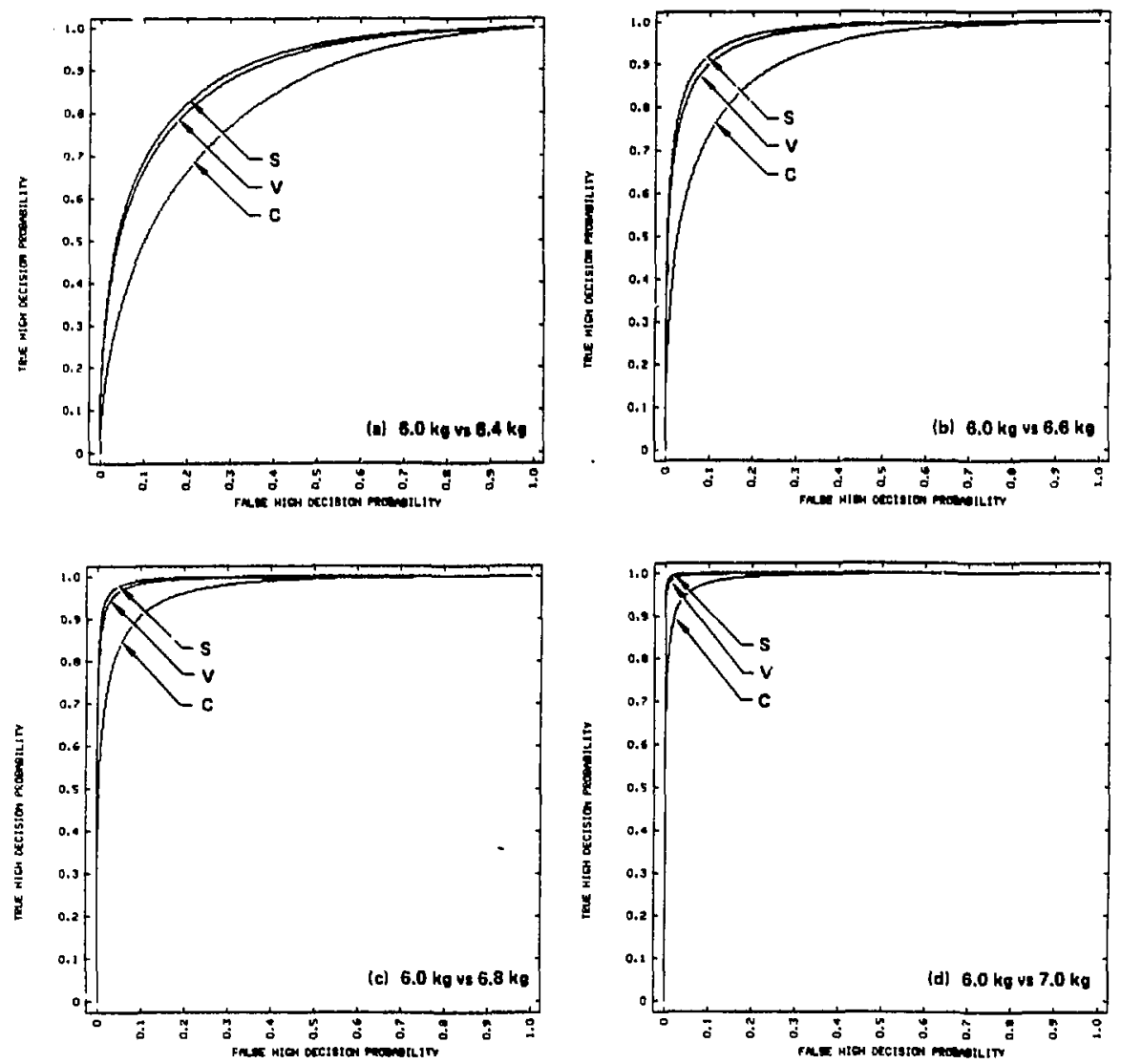

Fig. 34. Decision rule performance characteristics for a nonfission source, low fuel mass, 16 detectors active, and a full record of detections processed. $S$ is the serial-covariance-to-mean ratio curve; $\mathrm{V}$ is the variance-to-mean ratio curve; $\mathrm{C}$ is the covariance-to-mean ratio curve. Mass callouts identify the weak and strong signal sources; e.g., "6.0 kg vs 6.4 kg" means the lowmultiplication assembly is fueled with $6.0 \mathrm{~kg}$ of uranium and the high-multiplication assembly is fueled with $6.4 \mathrm{~kg}$ of uranium. 

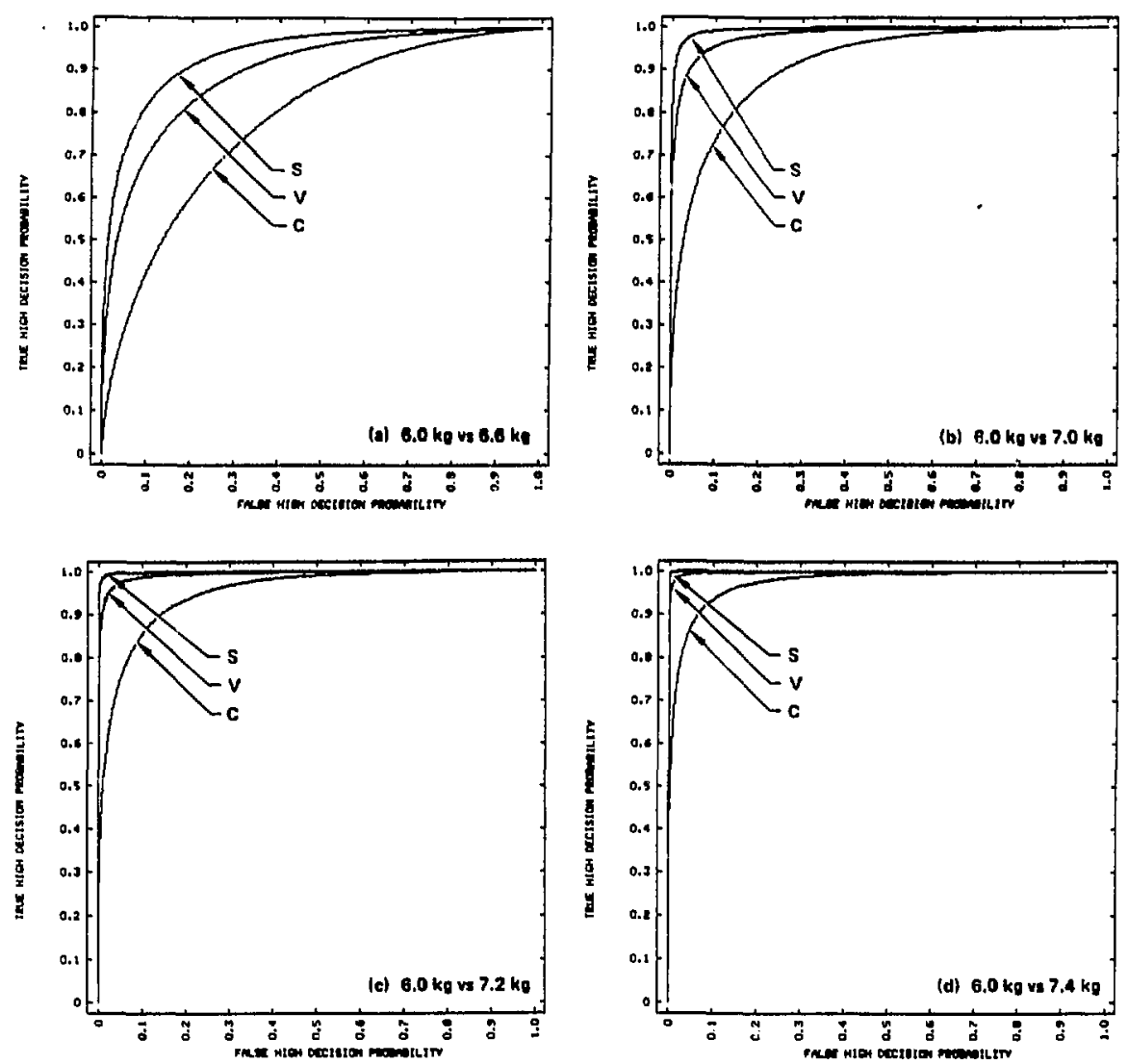

Fig. 35. Decision rule performance characteristics for a nonfission source, low fuel mass, 8 detectors active, and a full record of detections processed. $S$ is the serial-covariance-to-mean ratio curve; $V$ is the variance-to-mean ratio curve; $C$ is the covariance-to-mean ratio curve. Mass callouts identify the weak and strong signal sources; e.g., "6.0 kg vs $6.6 \mathrm{~kg}$ " means the lowmultiplication assembly is fueled with $6.0 \mathrm{~kg}$ of uranium and the high-multiplication assembly is fueled with $6.6 \mathrm{~kg}$ of uranium. 

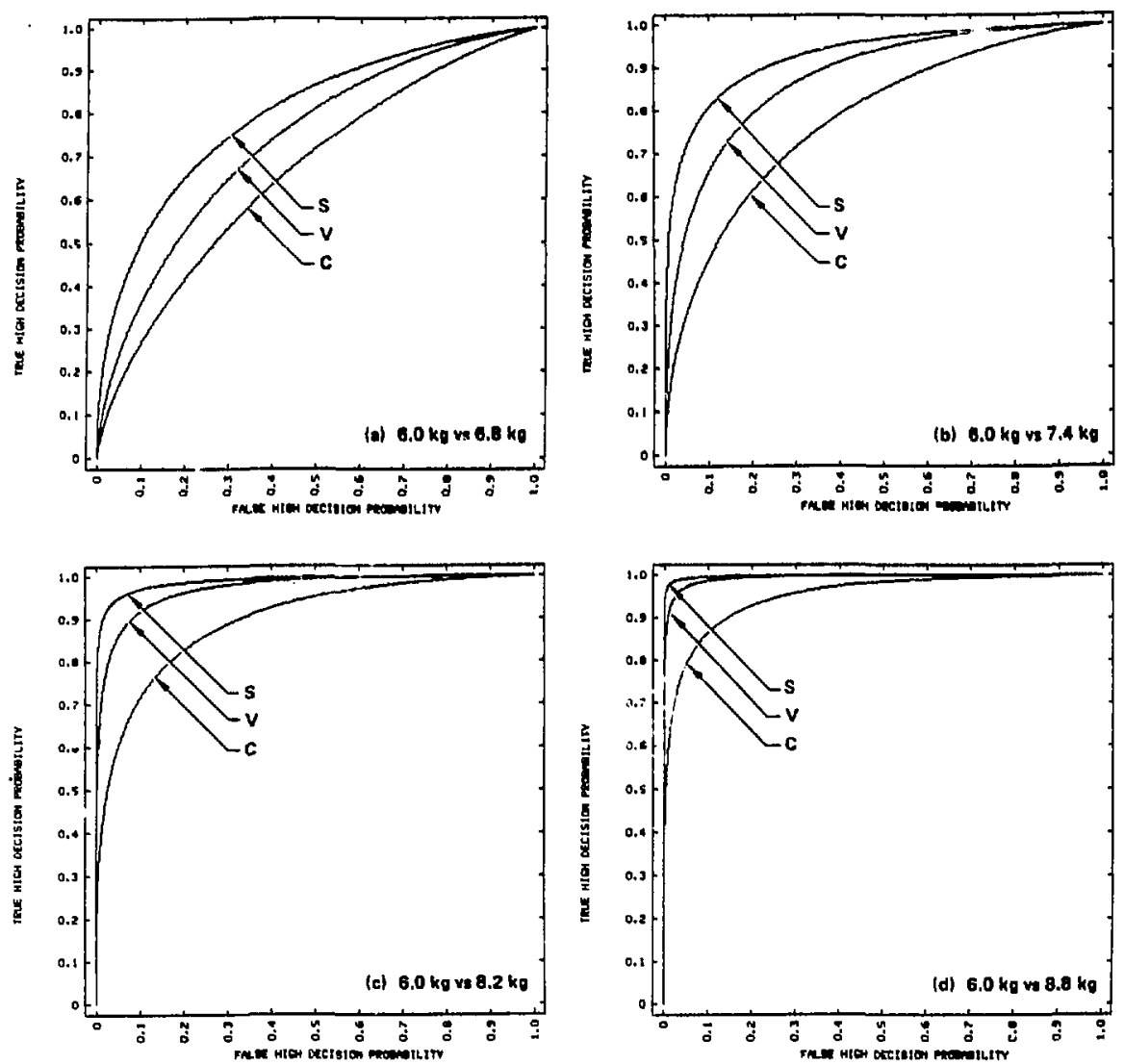

Fig. 36. Decision rule performance characteristics for a nonfission source, low fuel mass, 4 detectors active, and a full record of detections processed. $S$ is the serial-covariance-to-mean ratio curve; $V$ is the variance-to-mean ratio curve; $C$ is the covariance-to-mean ratio curve. Mass callouts identify the weak and strong signal sources; e.g., " $6.0 \mathrm{~kg}$ vs $6.8 \mathrm{~kg}$ " means the lowmultiplication assembly is fueled with $6.0 \mathrm{~kg}$ of uranium and the high-multiplication assembly is fueled with $6.8 \mathrm{~kg}$ of uranium. 

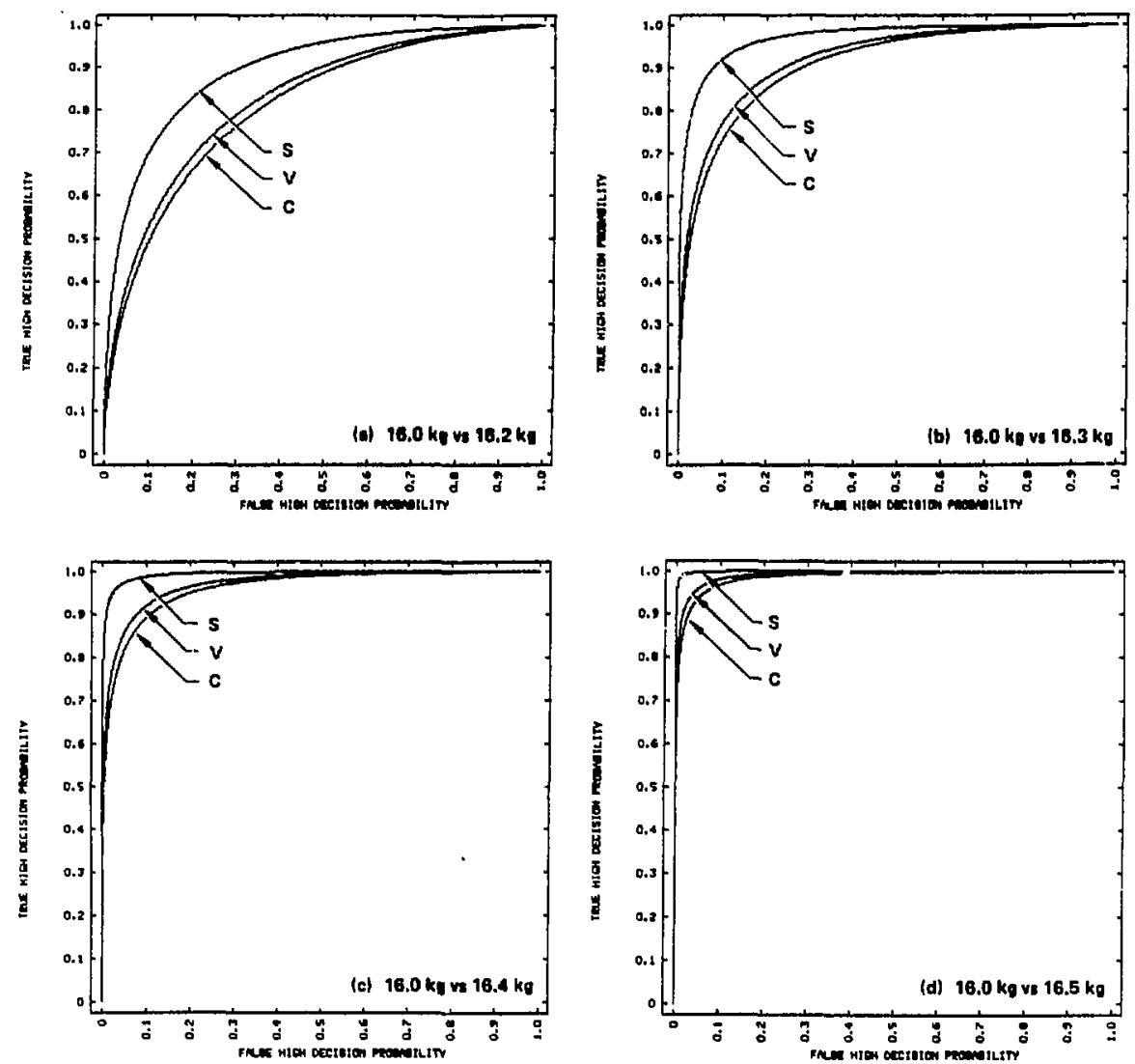

Fig. 37. Decision rule performance characteristics for a nonfission source, high fuel mass, 16 detectors active, and a full record of detections processed. $S$ is the serial-covariance-to-mean ratio curve; $V$ is the variance-to-mean ratio curve; $C$ is the covariance-to-mean ratio curve. Mass callouts identify the weak and strong signal sources; e.g., " $16.0 \mathrm{~kg}$ vs $16.2 \mathrm{~kg}$ " means the lowmultiplication assembly is fueled with $16.0 \mathrm{~kg}$ of uranium and the high-multiplication assembly is fueled with $16.2 \mathrm{~kg}$ of uranium. 

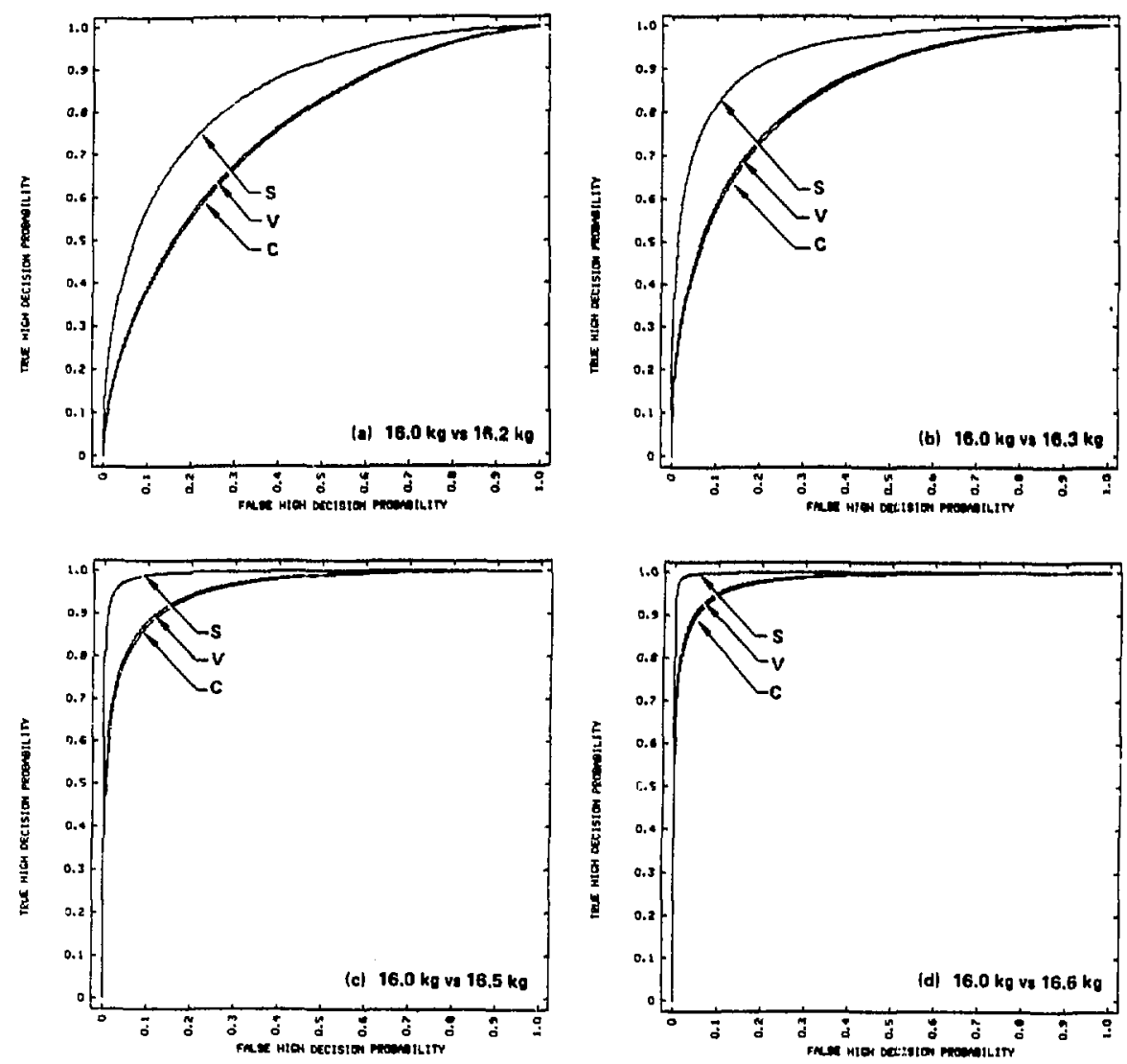

Fig. 38. Decision rule performance characteristics for a nonfission source, high fuel mass, 8 detectors active, and a full record of detections processed. $S$ is the serial-covariance-to-mean ratio curve; $\mathrm{V}$ is the variance-to-mean ratio curve; $\mathrm{C}$ is the covariance-to-mean ratio curve. Mass callov!s identify the weak and strong signal sources; e.g., " $16.0 \mathrm{~kg}$ vs $16.2 \mathrm{~kg}$ " means the lowmultiplication assembly is fueled with $16.0 \mathrm{~kg}$ of uranium and the high-multiplication assembly is fueled with $16.2 \mathrm{~kg}$ of uranium. 

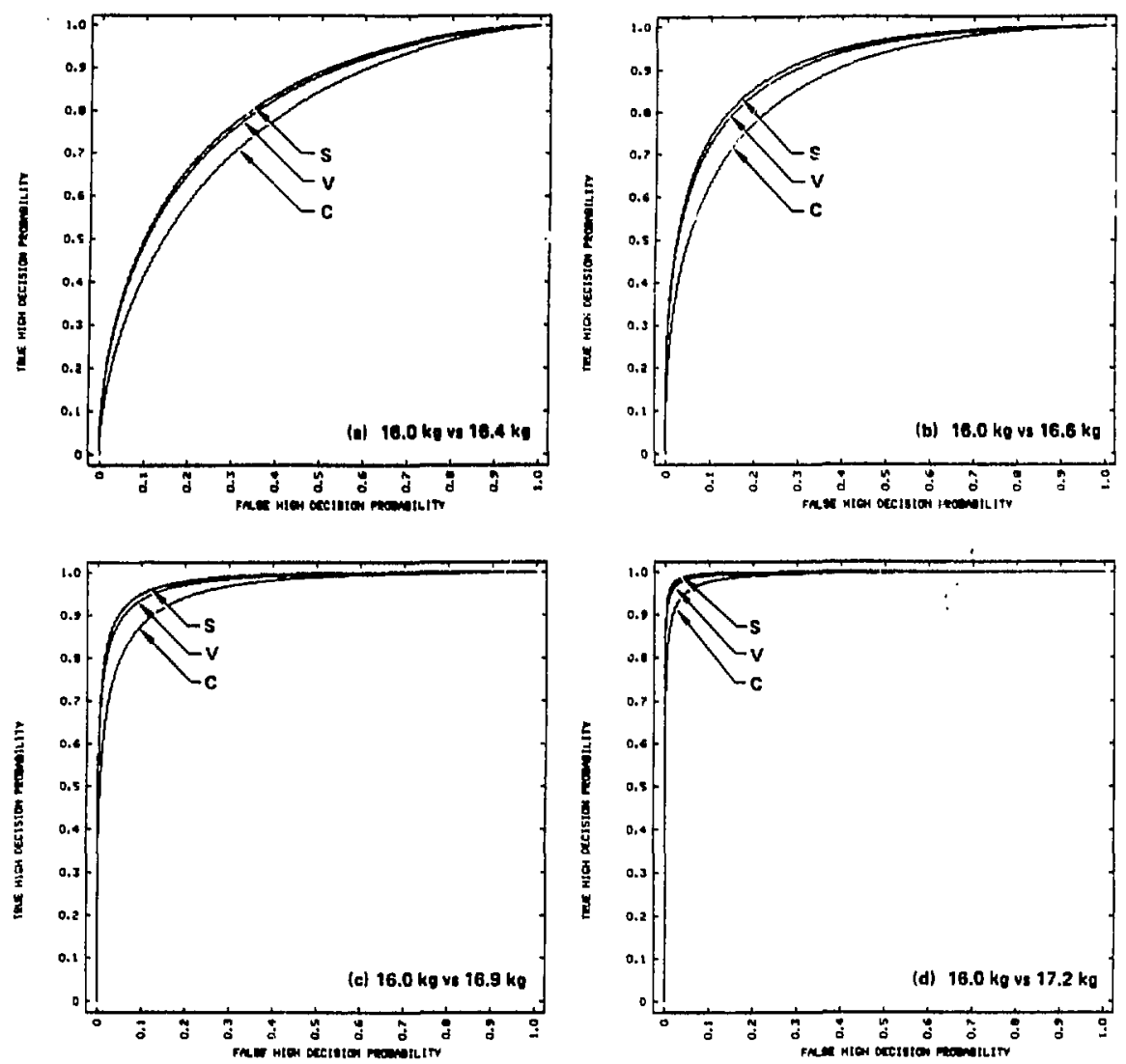

Fig. 39. Decision rule performance characteristics for a nonfission source, high fuel mass, 4 detectors active, and a full record of detections processed. $S$ is the serial-covariance-to-mean ratio curve; $\mathrm{V}$ is the variance-to-mean ratio curve; $\mathrm{C}$ is the covariance-to-mean ratio curve. Mass callouts identify the weak and strong signal sources; e.g., "16.0 kg vs $16.4 \mathrm{~kg}^{\prime}$ " means the lowmultiplication assembly is fueled with $16.0 \mathrm{~kg}$ of uranium and the high-multiplication asfembly is fueled with $16.4 \mathrm{~kg}$ of uranium. 

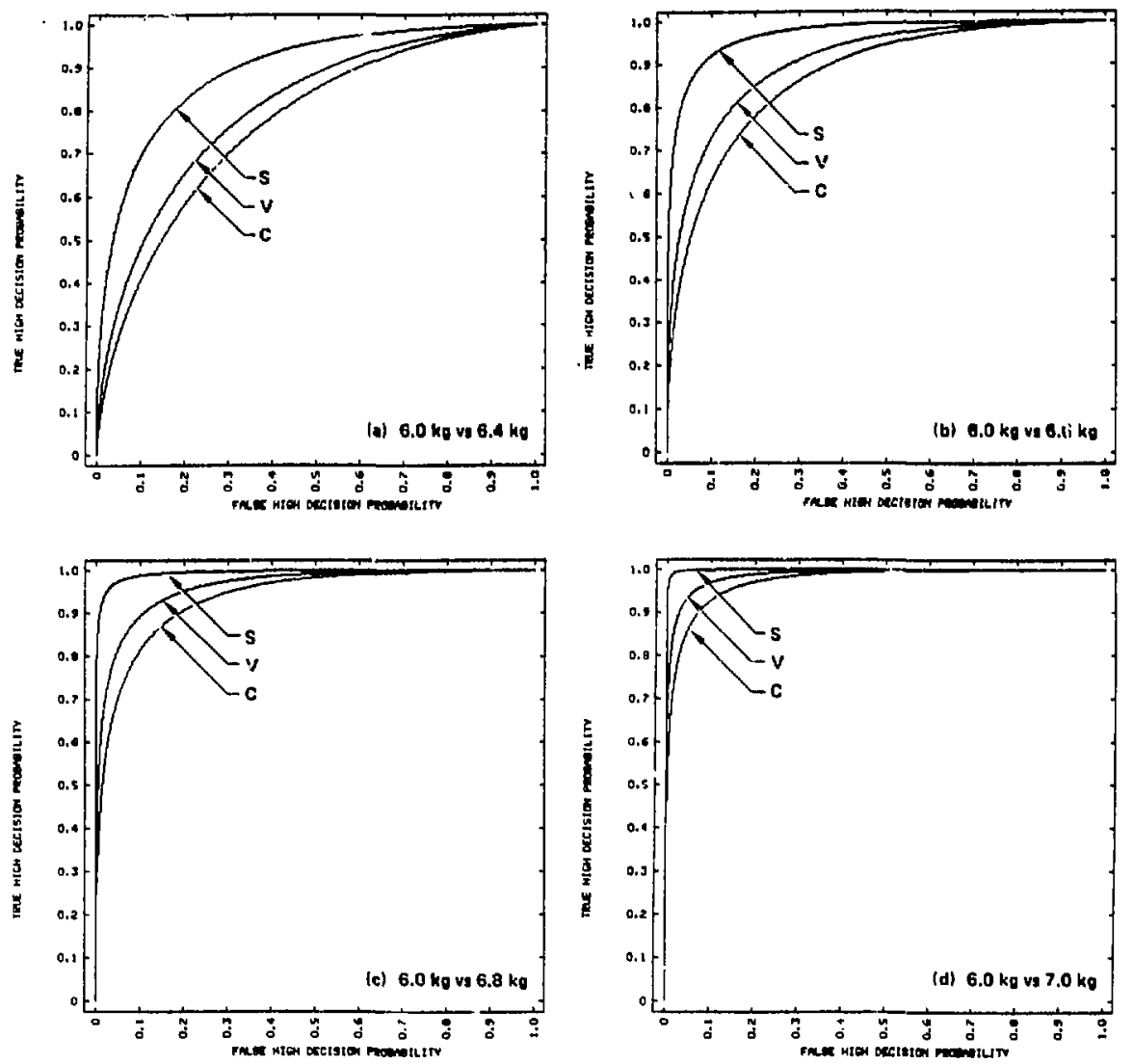

Fig. 40. Decision rule performance characteristics for a fission source, low fuel mass, 16 detectors active, and a full record of detections processed. $S$ is the serial-covariance-to-mean ratio curve; $V$ is the variance-to-mean ratio curve; $C$ is the covariance-to-mean ratio curve. Mass callouts identify the weak and strong signal sources; e.g., "6.0 kg vs 6.4 kg" means the lowmultiplication assembly is fueled with $6.0 \mathrm{~kg}$ of uranium and the high-multiplication assembly is fueled with $6.4 \mathrm{~kg}$ of uranium. 

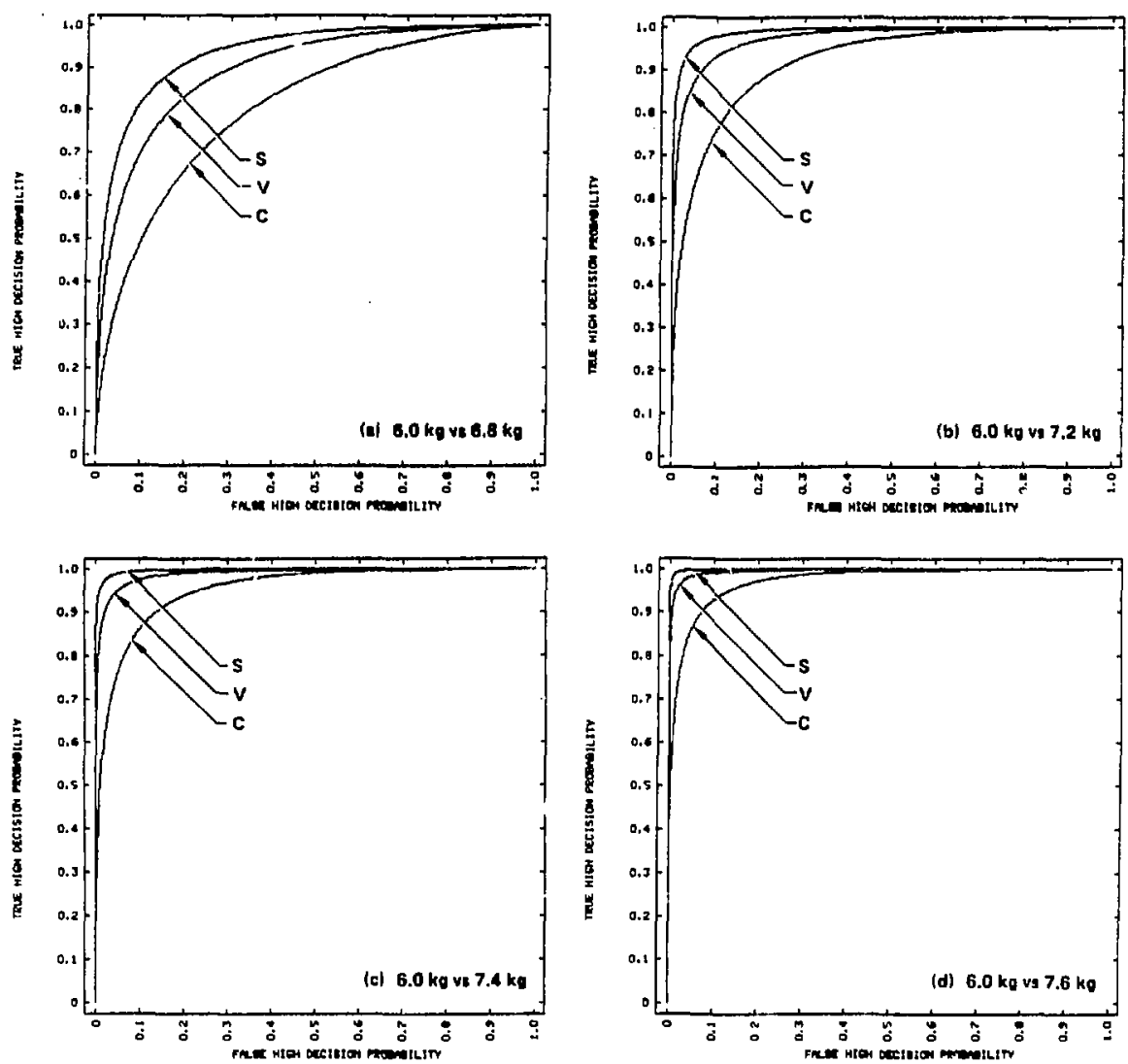

Fig. 41. Decision rule performance characteristics for a fission source, low fuel mass, 8 cietectors active, and a full record of detections processed. $S$ is the serial-covariance-to-mean ratio curve; $\mathrm{V}$ is the variance-to-mean ratiu curve; $C$ is the covariance-to-mean ratio curve. Mass callouts identify the weak and strong signal sources; e.g., "6.0 kg vs $6.8 \mathrm{~kg}$ " means the lowmultiplication assembly is fueled with $6.0 \mathrm{~kg}$ of uranium and the high-muliiplication assembly is fueled with $6.8 \mathrm{~kg}$ af uranium. 

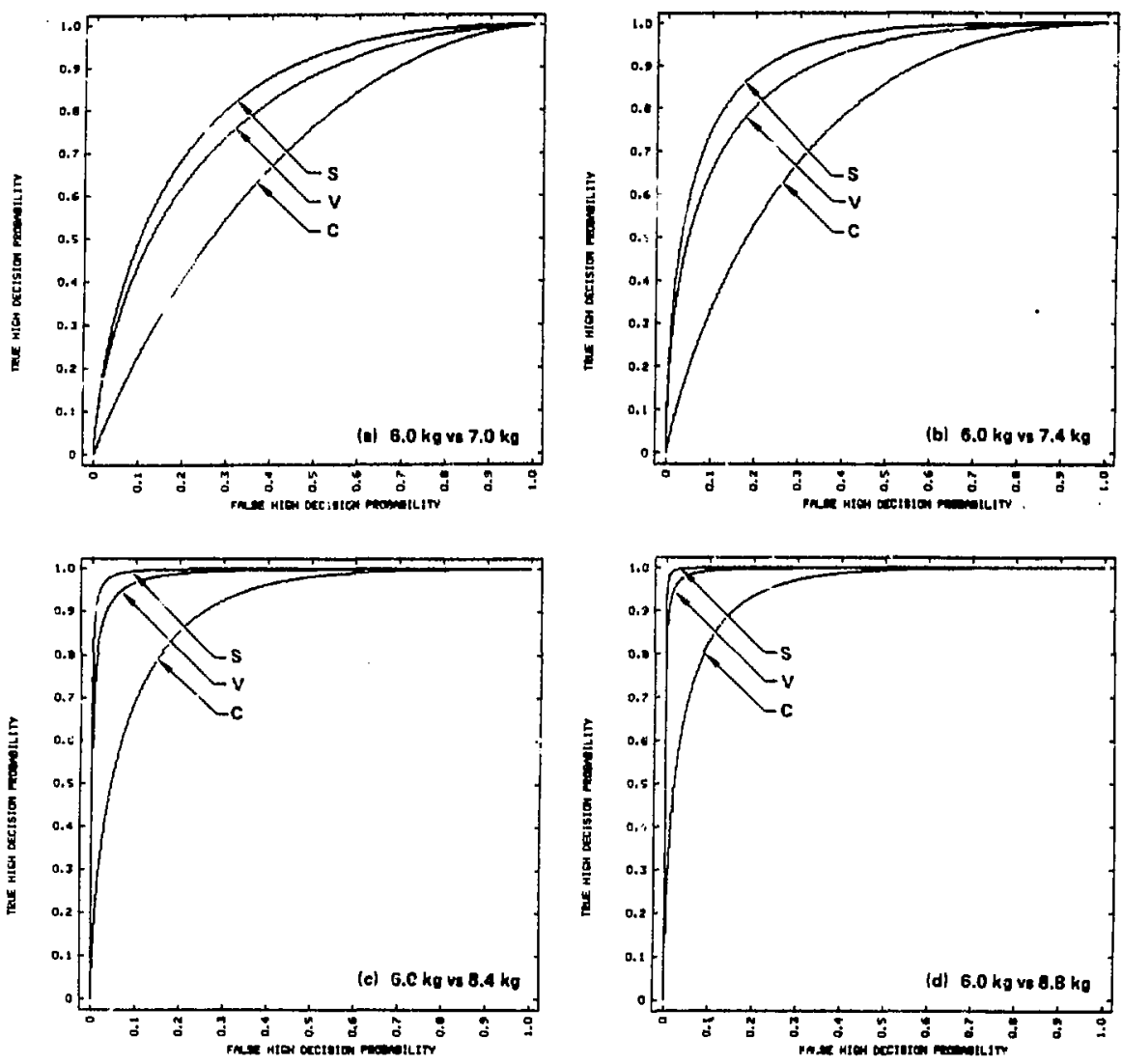

Fig. 42. Decision rule performance characteristics for a fission source, low fuel mass, 4 detectors active, and a full record of detections processed. $S$ is the serial-covariance-to-mean ratio curve; $\mathrm{V}$ is the variance-to-mean raito curve; $\mathrm{C}$ is the covariance-to-mean ratio curve. Mass callouts identify the weak and strong signal sources; e.g., "6.0 kg vs $7.0 \mathrm{~kg}$ " means the lowmultiplication assembly is fueled with $6.0 \mathrm{~kg}$ of uranium and the high-multiplication assembly is fueled with $7.0 \mathrm{~kg}$ of uranium. 

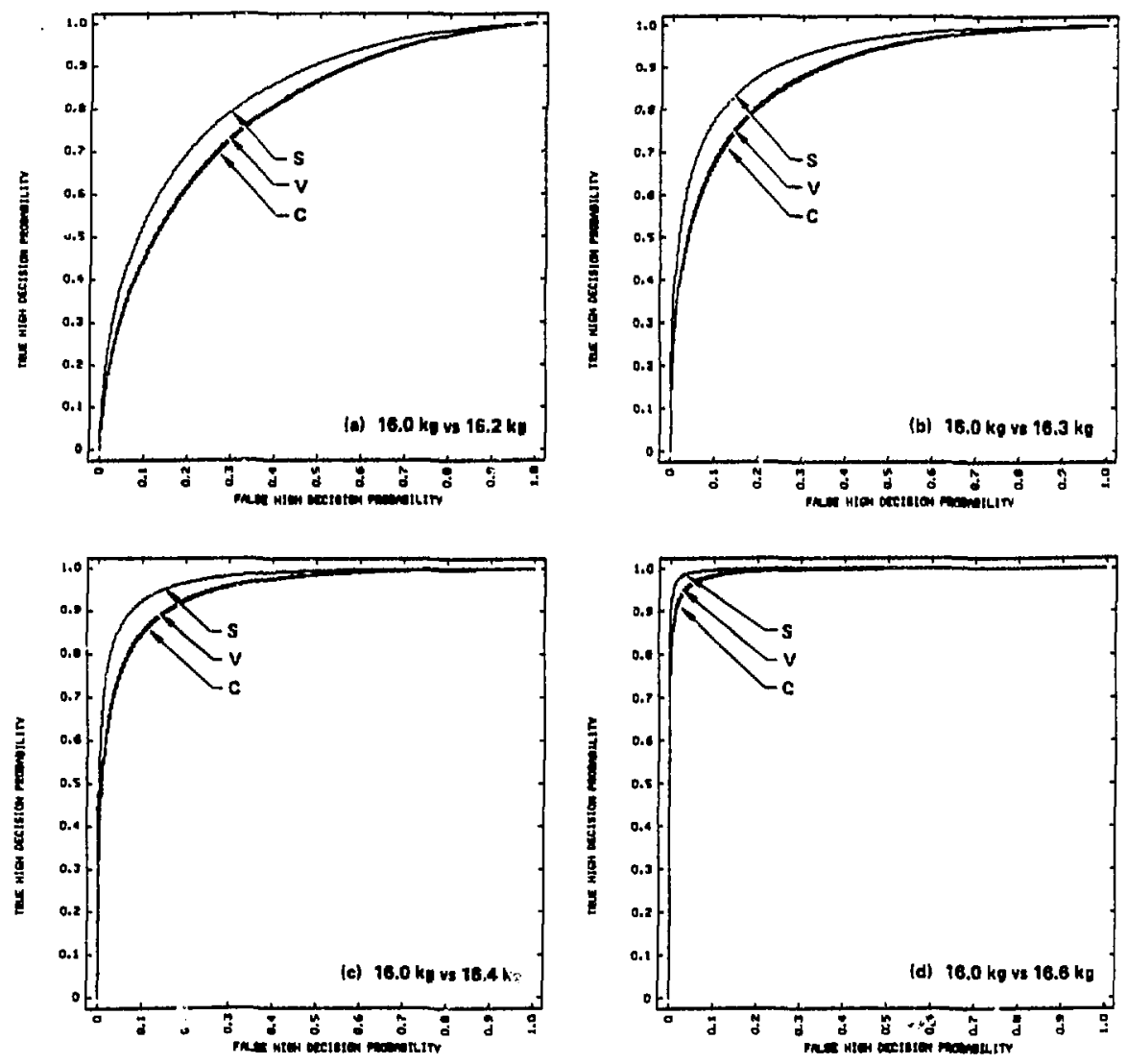

Fig. 43. Decision rule performance characteristics for a fission source, high fuel mass, 16 detectors active, and a full record of detections processed. $S$ is the serial-covariance-to-mean ratio curve; $V$ is the variance-to-mean ratio curve; $C$ is the covariance-to-mean ratio curve. Mass callouts identify the weak and strong signal sources; e.g., "16.0 kg vs $16.2 \mathrm{~kg}$ " means the lowmultiplication assembly is fueled with $16.0 \mathrm{~kg}$ of uranium and the high-multiplication assembly is fueled with $16.2 \mathrm{~kg}$ of uranium. 

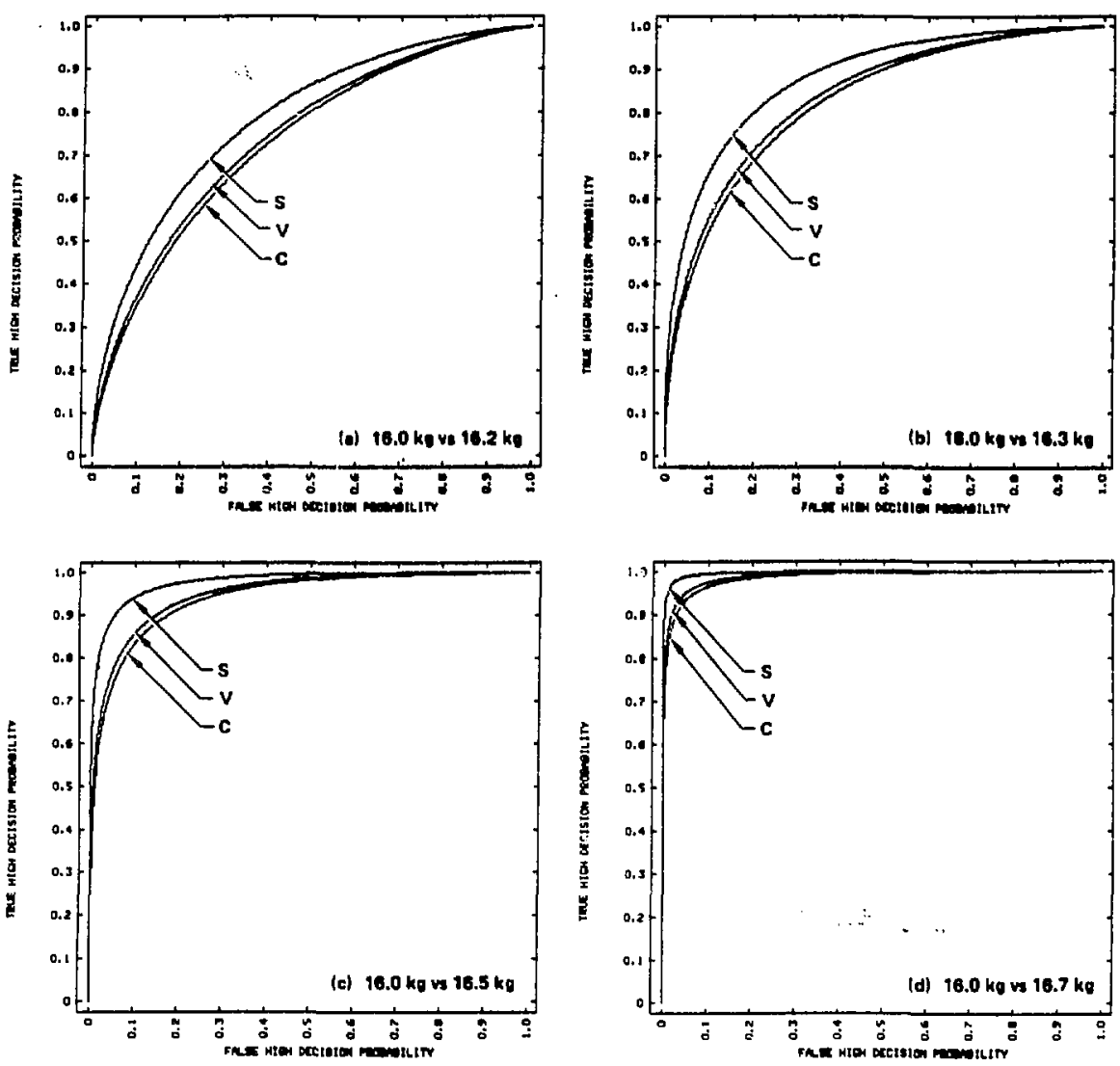

Fig. 44. Decision rule performance characteristics for a fission source, high fuel mass, 8 detectors active, and a full record of detections processed. $S$ is the serial-covariance-to-mean ratio curve; $\mathrm{V}$ is the variance-to-mean ratio curve; $\mathrm{C}$ is the covariance-to-mean ratio curve. Mass callouts identify the weak and strong signal sources; e.g." " $16.0 \mathrm{~kg}$ vs $16.2 \mathrm{~kg}$ " means the lowmultiplication assembly is fueled with $16.0 \mathrm{~kg}$ of uranium and the high-multiplication assembly is fueled with $16.2 \mathrm{~kg}$ of uranium. 

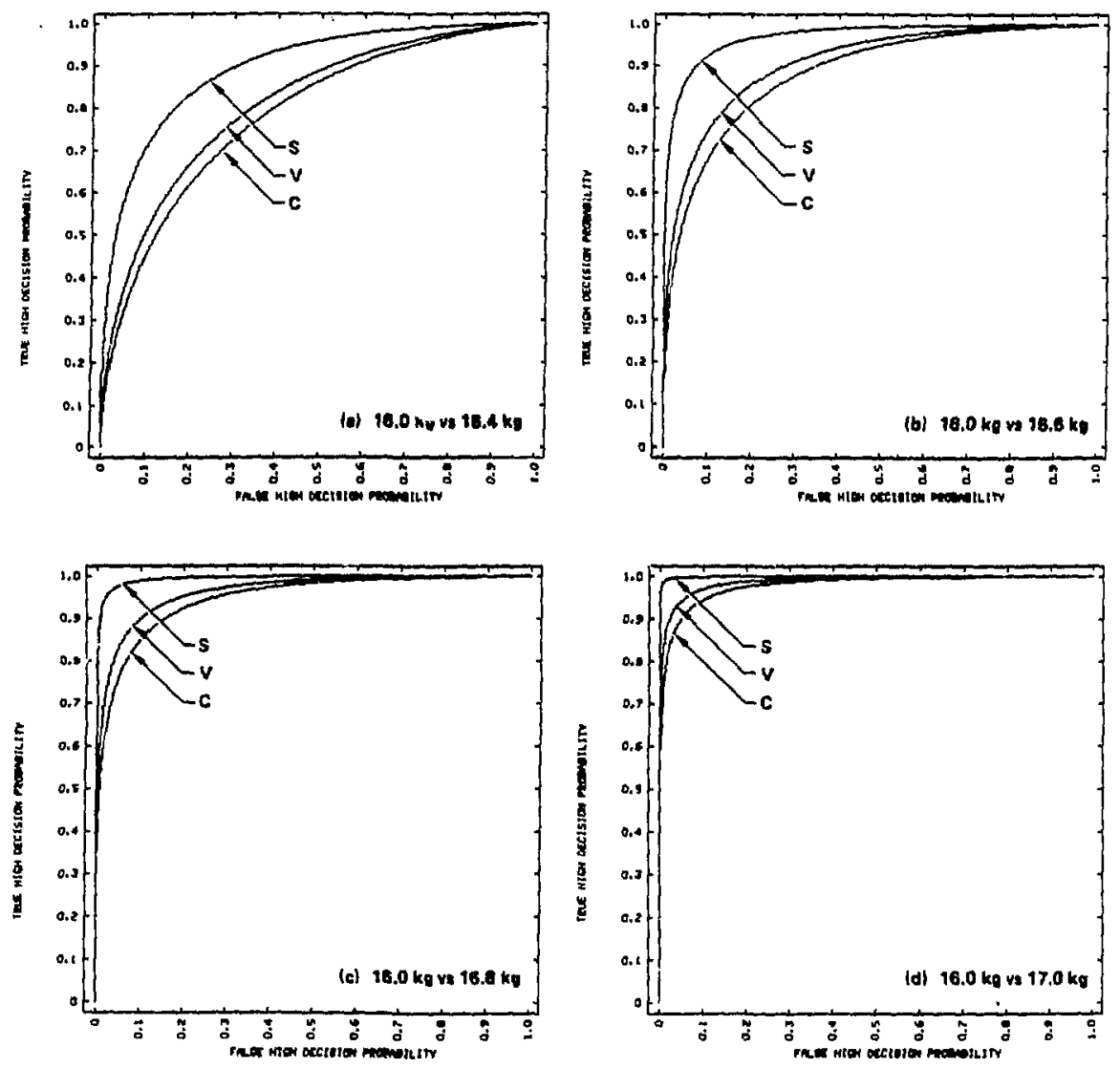

Fig. 45. Decision rule performance characteristics for a fission source, high fuel mass, 4 detectors active, and a full record of detections processed. $S$ is the serial-covariance-to-mean ratio curve; $\mathrm{V}$ is the variance-to-mean ratio curve; $\mathrm{C}$ is the covariance-to-mean ratio curve. Mass callouts identify the weak and strong signal sources; e.g., "16.0 kg vs $16.4 \mathrm{~kg}^{\prime \prime}$ means the lowmultiplication assembly is fueled with $16.0 \mathrm{~kg}$ of uranium and the high-multiplication assembly is fueled with $16.4 \mathrm{~kg}$ of uranium. 
To see how the curves were generated, focus attention on the curve labeled V in Fig. 34(a). The steps that led to that curve are as follows:

Step 1: From Fig. 9(a), curve A, I extract the mean value of $x(1)$ that corresponds to $6.0 \mathrm{~kg}$ of fuel: 0.234 . Similarly, I extract the mean value of $x(1)$ that corresponds to $6.4 \mathrm{~kg}$ of fuel: 0.257 .

Step 2: I determine measures of uncertainty for the two means found in Step 1. By a leastsquares polynomial interpolation of the uncertainties determined at each of the four fuel loadings I find a signal-to-noise ratio of 22 for the $6.0-\mathrm{kg}$ point and 24 for the $6.4-\mathrm{kg}$ point. By signal-to-noise ratio I mean (sample mean)/(sample standard deviation).

Step 3: I draw a DRPC as described earlier in this chapter, applying the following conditions. The processor output random variable, $X$, is nnrmally distributed with a mean of 0.234 and a signal-to-noise ratio of 22 if the input is from the weak generator, normally distributed with a mean of 0.257 and a signal-to-noise ratio of 24 if from the strong generator.

To summarize, the $V$ curve of Fig. 34(a) is the image of the map

$x-\left[T\left(\frac{x-v_{1}}{\sigma_{1}}\right), T\left(\frac{x-v_{2}}{\sigma_{2}}\right)\right]$,

where

$T(x)=-\frac{1}{\sqrt{2 \pi}} \int_{x}^{\infty} \mathrm{e}^{-\frac{1}{2} t^{2}} d t$

and

$i_{1}^{\prime}=0.234, \sigma_{1}=0.234 / 22=0.0106$,

$i_{2}=0.257, \sigma_{2}=0.257 / 24=0.0107$.

All 16-detector curves (Figs. 34, 37, 40, and 43) were generated by the technique just described. Variance-to-mean ratio data (Fig. 9) were used to generate the $V$ curves, covariance-to-mean ratio data (Fig. 11) to generate the $C$ curves, and serial-covariance-to-mean ratio data (Fig. 13) to generate the $\mathrm{S}$ curves. Ubvious changes generated the 8-detector and 4-detector curves.

A word about my choice of the normal distribution to describe the discriminator data is in order. First, a disclaimer. 1 do no: claim that the normal model is singularly appropriate. I have no a priori, theoretical reason to think so. I chose the normal law because it appears from visual inspection to represent the data fairly well and because it is familiar. Figure 46 shows two pieces of evidence: variance-to-mean ratio data for the 1-fuel-shell case and for the 4-fuel-shell case, with the assembly driven by the nonfission source. I assert that the other 70 cases are also reasonably well represented by the normai law.

Observe from Figs. 34 through 45 that the performances of the three discriminators are fairly consistently different but not greatiy so. The ordering that emerges is serial-covariance-to-mean ratio $Z$ variance-to-mean ratio $Z$ covariance-to-mean ratio.

These experiments do not support the conjecture that covariance-to-mean $(\mathrm{C} / \mathrm{M})$ techniques should be superior to variance-to-mean (V/M) techniques because $C / M$ techniques avoid estimating the uncorrelated Poisson variance-to-mean ratio.* Figures 34 through 45 show that the C/M discriminator is almost always the least effective of the three.

\footnotetext{
"This conjecture has been made by Nicola Pacilio in Reactor Noise Annlysis in the Time Domnin. U.S. Atomic Energy Commission/Division of Technical Information, TID-24512 (1969), p. 53, ind by Robert E. Uhrig in Random Nuise Techuninuts in Nuclear Rutuctor Systems (The Runald Press Company, New York, 1970), p. 78.
} 

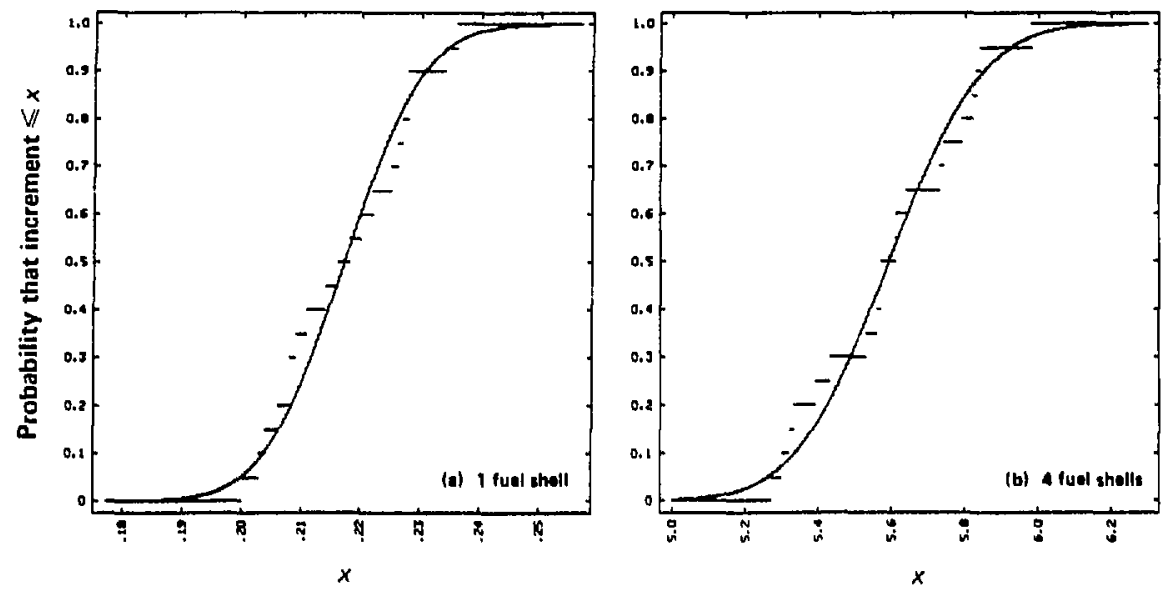

Fig. 46. Empirical distribution functions (EDF) constructed from asymptotic increments in the variance-to-mean ratio data. Each EDF represents a 20-sample data set. In both cases, the neutron source was nonfission and all 16 detectors were active. The smooth curves are normal cumulative distribution functions with means and standard deviations equal to the means and standard deviations of the underlying data sets. An EDF of a data sample of size $n$ is the cumulative distribution function that corresponds to a probability mass function with an alom of mass $1 / n$ located at each of the $n$ data points.

Table 2 summarizes the increments in fuel mass that are required for satisfactory discrimination between low-mass and high-mass situations. For example, the entry in row 1, column 1 $(1.0 \mathrm{~kg}$ ) says that with 16 detectors and a nonfission source, $6.0 \mathrm{~kg}$ and $7.0 \mathrm{~kg}(6.0+1.0)$ of fuel can be satisfactorily distinguished but 6.0 and $<7.0$ cannot. Table entries correspond to the best of the four DRPCs in each of Figs. 34 through 45.

Table 2. Increment in fuel mass, in kilograms, required to obtain satisfactory discrimination.

\begin{tabular}{|c|c|c|c|c|}
\hline \multirow{2}{*}{$\begin{array}{l}\text { Low mass } \\
\text { No. of } \\
\text { detectors }\end{array}$} & \multicolumn{2}{|c|}{ Nonfission source } & \multicolumn{2}{|c|}{ Fission source } \\
\hline & 6.0 & 16.0 & 6.0 & 16.0 \\
\hline 16 & 1.0 & 0.5 & 1.0 & 0.6 \\
\hline 8 & 1.4 & 0.6 & 1.6 & 0.7 \\
\hline 4 & 2.8 & 1.2 & 2.8 & 1.0 \\
\hline
\end{tabular}


Table 3 summarizes the same information for the multiplication factor, $k$. Entries display the value of $\Delta k$ that must be added to low $k$ if the best of the three discriminators is to satisfactorily separate low- $k$ and high- $k$ situations. Multiplication factors were calculated as explained in the Appendix. Table entries correspond to the best of the four DRPCs in each of Figs. 34 through 45.

We see from Table 3 that in the neighborhood of $k=0.6$ the $\Delta k$ required for satisfactory discrimination varies from about $3 \%$ to about $7 \%$ as the number of active detectors varies from 16 to 4 . In the neighborhood of $k=0.8$ the $\Delta k$ required for satisfactory discrimination varies from about $1 \%$ to about $2 \%$ as the number of active detectors varies from 16 to 4 .

Table 3. Increment in multiplication factor $(k)$, required to obtain satisfactory discrimination.

\begin{tabular}{c|cccr}
\hline & Low $k$ & Nisnfission source & \multicolumn{2}{c}{ Fission source } \\
$\begin{array}{l}\text { No. of } \\
\text { detectors }\end{array}$ & 0.605 & 0.819 & 0.605 & 0.819 \\
\hline 16 & & & & 0.010 \\
8 & 0.025 & 0.009 & 0.025 & 0.012 \\
4 & 0.035 & 0.010 & 0.040 & 0.017 \\
\hline
\end{tabular}




\section{Conclusions and Directions for Further Work}

I have studied the statistics of detected neutrons that leaked from four subcritical reflected, enriched-uranium assemblies, to explore the feasibility of developing a criticality warning system based on neutron noise analysis. I studied three possible discriminators, i.e., three signatures that might be used to discriminate among assemblies of various multiplications. They are (1) varianceto-mean ratio of the counts in a time bin (V/M), (2) covariance-to-mean ratio of the counts in a common time bin from two different detectors (C/M), (3) covariance-to-mean ratio of the counts from a single detector in two adjacent time bins of equal length, which I call the serial-covariancetu-mean ratio $(\mathrm{SC} / \mathrm{M})$. The performances of the three discriminators were not greatly different, but a hierarchy did emerge: $\mathrm{SC} / \mathrm{M} \geq \mathrm{V} / \mathrm{M} \geq \mathrm{C} / \mathrm{M}$. An example of some results: in the neighborhood of $k=0.6$ the $\Delta k$ required for satisfactory discrimination varies from about $3 \%$ to $7 \%$ as detector solid angle varies from $19 \%$ to $5 \%$. In the neighborhood of $k=0.8$ the corresponding $\Delta k$ are $1 \%$ and $2 \%$.

It was by using DRI'Cs as measures of goodness that l arrived at the performance order $\mathrm{SC} / \mathrm{M}$ $Z V / M \geq C / M$. The sample size of 20 on which each DRIPC is based is not large, so there is uncertainty in each. Nevertheless, 1 find it convincing that the stated order emerges in case after case. Here are two quantitative examples of relative performances of discriminators taken from DRPCs that appear in the main body of the text. Case l: nonfission source, 16 detectors, task is to discriminate between $16.0-\mathrm{kg}$ and $16.4-\mathrm{kg}$ assemblies, $5 \%$ false high decision probability accepted. Curresponding true high decision probabilities for $\mathrm{SC} / \mathrm{M}, \mathrm{V} / \mathrm{M}$, and $\mathrm{C} / \mathrm{M}$ discriminators are $97 \%, 86 \%$, and $81 \%$. Case II: same as Case 1 except assemblies are driven by a fission source. Corresponding true high decision probabilities for $\mathrm{SC} / \mathrm{M}, \mathrm{V} / \mathrm{M}$, and $\mathrm{C} / \mathrm{M}$ discriminators are $86 \%, 75 \%$, and $75 \%$.

That the variance-to-mean discriminator performed slightly better than the covariance-tomean discriminator is inconsistent with the lore that $\mathrm{C} / \mathrm{M}$ techniques should be better than ViM techniques because $\mathrm{C} / \mathrm{M}$ techniques avoid estimating the contribution of the Poisson component of the sample. The result suggests that for a given set of assets (a given number of detectors and associated electronics) it is better to lump the detectors into one unit and perform V/M measurements than to split the detectors into a pair of symmetric units and perform C/M measurements. Apparently, the reduction in detector efficiency that is a consequence of the splitting eliminates whatever advantage might otherwise accrue to $C / M$ measurements.

The newly introduced SC/M discriminator performed slightly better than either of the other two, perhaps because it embodies the advantages of both: detection efficiency of the V/M discriminator and avoidance of estimation of the Poisson component that is a property of the $\mathrm{C} / \mathrm{M}$ discriminator.

The discriminators I studied performed well enough in deeply subcritical situations to deserve testing in an applications environment. They have a good chance of detecting changes in reactivity that are potentially dangerous. One can expect sharpest results when doing comparisons, i.e., when comparing two records, one taken in the past under circumstances known to be normal and one taken now to search for change. Further, to be most effective, the geometry of the past and present arrangements should be as nearly identical as possible. In particular, the detector positions relative to the center of the zone being surveyed should be faithfully reproduced if possible. The situation is analogous to that of a physician reading two electrocardiograms, one taken when the patient was known to possess normal health and one taken later to try to detect change.

I have stressed the fact that none of the discriminators studied depended upon gross count rate. However, I do not advocate completely ignoring gross count rate. Particularly in continuous monitoring applications, gross count rate might serve as a useful warning signal. With experience, an operator could learn the normal range of variation of count rate at a given work station. A count rate well above that range could serve as a warning to look deeper into the situation before resuming business as usual. Noise analysis techniques could supply that deeper look.

In contemplating possible applications, it is of interest to get some idea of the cycle time that might be required by a field instrument, that is, the time from start of data collection to the decision of whether or not the reactivity has increased significantly since the last interrogation. I visualize a system (detectors, electronics, and small dedicated computer) that is capable of collecting, storing, transmitting, and processing data. The operator can perform on-the-spot calculations for quick 
evaluation, and the data can be transmitted to a central recording and computing facility for more refined calculations and for permanent storage.

The apparatus I used for the work reported here was designed for exploratory laboratory measurements rather than for field application. No attempt was made to process data at maximum possible speed. Nevertheless, some idea of an upper bound for the cycle time of a field instrument can be obtained from experience with the laboratory instrument.

The LSI $11 / 23$ computer that is a component of the data-collection and recording system was programmed to perform on-the-spot check calculations. The program calculates variance-to-mean and covariance-to-mean ratios for a sequence cit time bin widths: $h, 2 h, \ldots, n h$. The operator selects $h$ and $n$. Typically, about 15 minutes were required for a calculation under the following condiing conditions: $h=250 \mu \mathrm{s}, \|=10$, count rate $=$ a few $\mathrm{kHz}$, total count $\sim 120,000$.

Calculations on the CDC 7600 machine were much faster. A fraction of a minute was sufficient for the calculation specified in the previous paragraph. The controlling time for the off-line, CDC $76(1)$ ) calculation was data transmission time. The link available required about 10 minutes for one disk of data $(-120,001)$ counts $)$ to be transmitted.

So, experience with the laboratory system suggests cycle times between 10 and 20 minutes. Probably, a carefully designed field instrument could do considerably better. For most applications, the cycle time under discussion is unlikely to be the controlling time. For periodic monitoring applications, instrument set-up and checkout time is apt to dominate. However, computing cycle time could be important in continuous monitoring applications.

Several directions tor further work come to mind if one were to launch a program to develop a criticality warning system. I discuss tive such directions.

An attractive feature of noise analysis discrimination techniques is their potential for being essentially passive and noninterfering. That potential can be literally realized only if the assembly being interrogated is self-excited, that is, comes equipped with an inherent neutron source. Hence, measurements on plutonium systems would be informative (since spontaneous fission rates of the even-even isotopes are significant). Likewise, assemblies containing intimate mixtures of uranium or plutonium and some of the lighter elements $[(\alpha, n)$ reactions] would be interesting candidates for measurements.

Lowering of discriminating power by extraneous background plagues all the methods studied with approximately equal force. Hence, a study of techniques for enhancing the ratio of assembly neutron count rate to extraneous neutron count rate would be worthwhile. The task is not easy since neutrons are notorious tor getting into everything, and no single technique is apt to be successful. However, a collection of methods, each applicable in special field circumstances, might be useful. Judicious arrangements of moderator slabs and sheets of cadmium come to mind.

Chemical processing plants are promising areas for application of criticality monitoring techniques. Hence, measurements on moderated, licjuid systems are needed. One might find here that delayed neutron effects are important, even for the low multiplications that are pertinent to criticality safety applications.

Field instruments would need a larger dynamic range and more flexibility than those possessed by the laboratory instrument used for this study. Hence, if a criticality warning system program were launched, it would have to include a comprehensive instrument-development program that would take advantage of advanced counting and computer technology.

Finally, it would almost certainly be worthwhile to conduct some measurements in the field before advanced instruments are developed, even with clumsy equipment. The difference between field and laboratory environments is apt to be substantial, and experiencing that difference early could yield valuable information for a development program. 


\section{Acknowledgments}

My sincere thanks to:

Howard Spracklen for application of his electronic wizardry to design, fabrication, and checkout of the data-collection system.

Mary Ann Mansigh for writing excellent programs to make the CDC 7600 computers do what I wanted them to do plus more.

Van Jackson and John Salisbury for helping with the experiments and running endless computer problems to reduce and analyze data.

O. Clinton Kolar for encouragement and arrangement of financial support throughout the enterprise.

My wife, Judith, for many things, none of which have anything to do with electronics, conputers, experiments, data, or money. 


\section{References}

1. F. de Hoffmann, "Statistical Aspects of Pile Theory," in The Science and Engineering of Nuclear Power, Vol. II, Clark Goodman, Ed. (Addison-Wesley Publishing Co., Inc., Cambridge, MA, 1949), Chap. 9.

2. E. Fermi, R. P. Feynman, and F. de Hofimann, Theory of the Criticality of the Water Boiler and the Determination of the Number of Delayed Neutrons, Los Alamos Scientific Laboratory, Los Alamos, NM, USAEC Report MDDC-383 (LADC-269) (1944).

3. F. de Hoffmann, Intensity Fluctuations of a Neutron Chain Reaction, Los Alamos Scientific Laboratory, Los Alamos, NM, USAEC Report MDDC-382 (LADC-256) (1946).

4. R. reyman, F. de Hoffmann, and R. Serber, "Dispersion of the Neutron Emission in U"' Fission," I. Nucl. Energy 3, 64 (:956).

5. J. D. Orndoff, "Prompt Neutron Periods of Metal Critical Assemblies," Nucl. Sti. Eng. 2 (4), 450 (1957).

6. N. Pacilio et al. "The Analysis of Reactor Noise: Measuring Statistical Fluctuations in Nuclear Systems," in Atumces in Nuclear Science and Tedmolegy, Volume II, E. J. Henley, J. Lewins, and M. Becker, Eds. (Plenum l'ress, New York, 1979), p. 67.

7. R. E. Uhrig, Ed., Noist' Analysis in Nuclear Systems, U. S. Atomic Energy Commission, TID-7679 (1964).

8. R. E. Uhrig, Ed., Neutron Neise, Waves and Pulse Propagation, U. S. Atomic Energy Commission, CONF-660206 (1967).

9. Statistical Methods in Experimental Reactor Kinetics and Related Techutiques, Proceedings, Petten, 1967, RCN-98 (1968).

10. Proc. Japan-United States Seminar on Nuclear Renctor Noise Analysis, Tokyo and Kyoto, 1968 (1968).

11. N. Pacilio, V. M. Jorio, and A. Columbino, Eds., "From Critical Assemblies to Power Reactors," Amm. Nucl. Energy 2 (2-5) (1975).

12. W. Seifritz and D. Stegemann, "Reactor Noise Analysis," At. Energy Rev. 9 (1), 129 (1971).

13. K. Saito, "On the Theory of Power Reactor Noise," Anm. Nucl. Sci. Eng. 1, 31, 107, 209 (1974).

14. J. A. Thie, Reactor Noise (Rowman \& Littlefield, New York, 1963).

15. N. Pacilio, Reactor Noise' Analysis in the Time Domain, U. S. Atomic Energy Commission, TID24512 (1969).

16. R. E. Uhrig, Rantom Noise Technitues in Nuclear Reattor Systems (Ronald Press Co., New York, 1970).

17. M. M. R. Williams, Random Processes in Nuclear Reactors (Perganon Press, Ltd., Oxford, 1974).

18. Joseph A. Thie, Power Renctor Noise (American Nuclear Society, La Grange Park, IL, 1981).

19. E. F. Bennett, "The Rice Formulation of Reactor Noise," Nucl. Sci. Eng. 8 (1), 53 (1960).

20. A. I. Mogilner and V. G. Zolotukhin, "The Staristical r-Method of Measuring the Kinetic Parameters of a Reactor," At. Encrgy (USSR) 10, 377 (1961).

21. D. Babala, "Point-Reactor Theory of Rossi-Alpha Experiment," Nucl. Sci. Eng. 28 (2), 237 (1967).

22. D. Babala, "Interval Distribution in Neutron Counting Statistics," Nucl. Sci. Eng. 28 (2), 243 (1967).

23. D. Babala and R. Orgin, "Measurement of the Prompt Neutron Decay Constant of the NORA Reactor by the Interval Distribution Technique," Nucl. Sci. Eng. 29 (2), 367 (1967).

24. Proc. Intem. Symp. Nuclear Safeguards, Viema, 1978, IAEA-SM-231/69 (International Atomic Energy Agency, Vienna, 1978). 
25. Proc. Ann. Symp. Safeguards and Nuclear Materials Management of ESARDA, 1st, Brussels, 1979 (European Safeguards R\&D Association, 1979).

26. Proc. Ann. Symp. Safeguards and Nuclear Materials Management of ESARDA, 2nd, Edinburgh, 1980 (European Safeguards R\&D Association, 1980).

27. E. J. Dowdy, C. N. Henry, A. A. Robba, and J. C. Pratt, "New Neutron Correlation Measurement Techniques for Special Nuclear Material Assay and Accountability," in Proc. Intern. Symp. Nuclear Safeguards, Viema, 1978, IAEA-SM-231/69 (International A tomic Energy Agency, Vienna, 1978).

28. E. J. Dowdy, G. E. Hansen, A. A. Robba, and J. C. Pratt, "Effects of $(\alpha, n)$ Contaminants and Sample Multiplication on Statistical Neutron Correlation Measurements," in Proc. Ann. Symp. Safcguards and Nuclear Materials Manngement of ESARDA, 2nd, Edinburgh, 1980 (European Safeguards R\&D Association, 1980).

29. J. T. Mihalczo, "The Use of Californium-252 as a Randomly Pulsed Neutron Source for Prompt-Neutron Decay Measurements," Nucl. Sci. Eng. 53 (4), 393 (1974).

30. J. T. Mihalczo, V. K. Pare, G. L. Ragan, M. V. Mathis, G. C. Tillett, "'Determination of Reactivity from Power Spectral Density Measurements with "'Cf," Nucl. Sci. Eng. 66 (1), 29 (1978).

31. W. T. King and J. T. Mihalczo, "Power Spectral Density Measurements with ":Cf for a LightWater-Moderated Research Reactor," Trans. Amer. Nacl. Suc. 33, 796 (1979).

32. J. T. Mihalczo, R. C. Kryter, and W. T. King, "Preliminary Investigation of "Cf-Driven Neutron Noise Analysis for Subcritical Fuel Solution Systems," Trans. Amer. Naci. Sci. 38, 359 (1981).

33. J. T. Mihalczo, "A Review of Subcriticality Measurements Using ":Cf-Driven Power Spectral Density Measurements," Trans. Amer. Nucl. Soc. 39, 517 (1981).

34. J. T. Mihalczo, W. T. King, and J. A. Renier, "Feasibility of LWR Subcritica Reactivity Monitoring Using the ":Cf-Driven Neutron Noise Method," Trans. Amer. Nucl. S $x$. 41, 619 (1982).

35. J. T. Mihalczo, and W. T. King, "'Cf-Source-Driven Neutron Noise Nethod for Measuring Subcriticality of Submerged HFIR Fuel Elements," Trans. Amer. Nucl. So:. 43, 408 (1982).

36. J. T. Mihalczo, "Benchmarking Criticality Safety Calculations with Subcritical Experiments," Trans. Amer. Nucl. Soc. 46, 451 (1984).

37. J. T. Mihalczo, W. T. King, E. B. Johnson, and E. D. Blakeman, "Subcriticality Measurements for a Fuel Solution Tank with Changing Fuel Concentration Using " $\mathrm{Cf}$-Source-Driven Neutron Noise Analysis," Trans. Amer. Nucl. Sci. 45, 337 (1983).

38. W. T. King, J. T. Mihalczo, and E. D. Blakeman, "Preliminary Investigation of the :"Cf-SourceDriven Noise Analysis Method of Subcriticality Measurement in LWR Fuel Storage and Initial Loading Applications," Trans. Amer. Nucl. Sci. 47, 239 (1984).

39. J. T. Mihalczo, W. T. King, and E. D. Blakeman, " $\mathrm{Cf}$-Source-Driven Neutron Noise $A$.nalysis Measurements for Coupled Urarium Metal Cylinders," Traris. Amer. Nucl. Sci. 49, 241 (1985).

40. A. J. Lindeman and L. Ruby, "Subcritical Reactivity from Neutron Statistics," Nucl. Sci. Eng. 29 (2), 308 (1967).

41. A. Szeless and L. Ruby, "Subcritical Reactivity from Reactor Noise," Nucl. Sci. Eng. 44 (1), 110 (1971).

42. M. M. El-Zeftawy and L. Ruby, "Addendum to Note on Subcritical Reactivity from Reactor Noise," Nucl. Sci. Eng. 47 (3), 370 (1972).

43. Hsiao-Kang Wang and L. Ruby, "Subcritical Reactivity as Determined from Reactor Noise," Nucl. Sci. Eng. 56 (2), 211 (1975).

44. Hsiao-Kang Wang and L. Ruby, "Some Additions to Reactor Noise Theory Which Include Delayed Neutrons," Nucl. Sci. Eng. 57 (1), 86 (1975). 
45. Tai-Ping Lung and L. Ruby, "Subcritical Reactivity from Polarity Correlation," Nid. Sci. Eng. 59 (4), 436 (1976).

46. Tai-Ping Lung and L. Ruby, "A More Direct Metl /od for Determining Subcritical Reactivity," Trans. Amer. Niud. Soc. 27, 944 (1977).

47. P. G. Hoel, S. C. Port, and C. J. Stone, Introduction to Probability Theory (Houghton Mifflin Company, Bosíon, 1971).

48. F. G. Huel, 5. C. Port, and C. J. Stone, Introduction to Stochastic Processes (Houghton Mifflin Company, Boston, 1971).

49. J. Lewins, Nucicar Reactor Kintetics and Control (Pergamon Press, Oxford, 1978) Chap. 6.

50. A. T. Bharucha-Reid, Elcments of the Theory of Markov Processes tind Their Applications (McGraw Hill, New York, 1960).

51. F. C. Courant and P. R. Wallace, "Fluctuations of the Number of Neutrons in a Pile," Phys. Rev. 72, 1038 (1957).

52. D. R. Cox and P. A. Lewis, The Statistical Analysis of S'ries of Events (Methuel, : i idon, 1966).

53. A. D. Whalen, Detection of Sighals in Noise (Academic Press, New York, 1971).

54. A. S. Zolnay, C. S. Barnett, and H. P. Spracklen, "Passive Neutron Multiplication Measurements," IEEE Trans. Nuil. Sit. NS-30 (1), 811 (1983). 


\section{Appendix. Experimental Appraratus}

My experimental apparatus consists of two systems: the multiplying assembly, a subcritical assembly of enriched uranium that is used to generate neutrons from different fuel configurations; and the data-collection and recording system, which collects all data for subsequent processing by a variety of algorithms.

\section{Multiplying Assembly}

The subcritical assembly consists of spherically symmetric shells of enriched uranium, internally and externally reflected with acrylic plastic. When the assembly is fully fueled, the mass of uranium in place is approximately $23 \mathrm{~kg}$. Figure A-1 displays a sketch of the essentials of the assembly. Fuel and reflector components are hemispherical shells that nest snugly together. There are four pairs of fuel shells, three pairs of internial reflector shells (also called moderators), and three pairs of external reflector shells. Table A-1 shows dimensions and masses of the fuel shells. The acrylic resin moderator shells have a common outside diameter of $11.8 \mathrm{~cm}$ and varying inside diameters to yield spherical shells of masses 331,539 , and $700 \mathrm{~g}$. The acrylic resin reflectors fit snugly around the largest uranium : ihell to provide $2-, 5-$, and $10-\mathrm{cm}$-thirk reflectors. Several combinations of these components may be assembled to obtain arrangements with different reactivities.

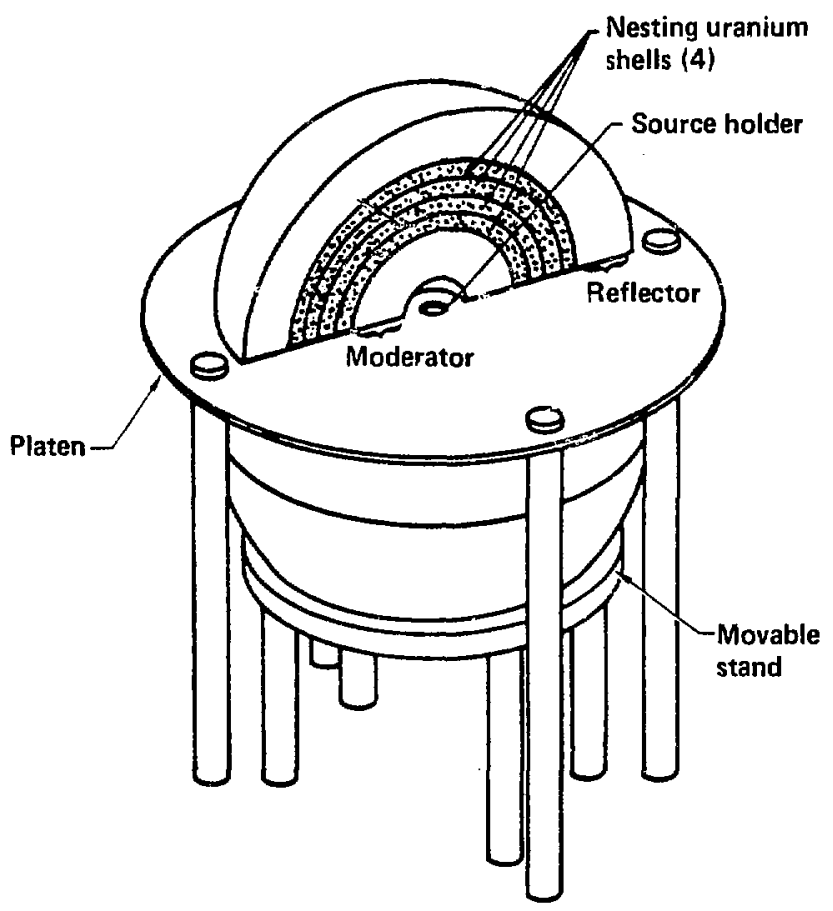

Fig. A-1. Subcritical assembly. Top hemishells are sectioned to show detail. 
Table A-1. Enriched uranium parts for multiplying assembly.

\begin{tabular}{cccc}
\hline $\begin{array}{c}\text { Hemispherical } \\
\text { shell }\end{array}$ & $\begin{array}{c}\text { Cuier } \\
\text { diameter (cm) }\end{array}$ & $\begin{array}{c}\text { Shell } \\
\text { thicknesa (cm) }\end{array}$ & Mass (g) \\
\hline 1 A & 13.1 & 0.63 & 2746 \\
1 B & 13.1 & 0.63 & 2747 \\
2 A & 14.2 & 0.55 & 3096 \\
2 B & 14.2 & 0.55 & 3095 \\
3 A & 15.0 & 0.40 & 2321 \\
3 B & 15.0 & 0.40 & 2313 \\
4 A & 15.9 & 0.45 & 3169 \\
4 B & 15.9 & 0.45 & 3362 \\
\hline
\end{tabular}

The assembiy machine consists of a fixed stand that supports a $0.15-\mathrm{cm}$-thick stainless steel platen, and a movable inner stand positioned below the fixed stand. The platen has a hole punched in its center to accommodate a neutron source.

An assembly sequence goes somewhat as follows: (1) assemble selected components on lower movable stand, (2) mount platen on fixe:i standards, (3) assemble matching components on the platen, (4) partially raise the lower stand, leaving enough space for the source, (5) insert neutron source, (6) raise lower stand until it mates with the platen, (7) take data.

The apparent leakage multiplication of the maximum reactivity configuration is -10 (Fig. A-2); tence remote assembly is not required.

Figure A-3 is a graph of calculated multiplication factor vs mass of fuel in the assembly. Calculations were performed for the one-, two-, three- and four-fuel-shell cases. The corresponding masses of $93.2 \%$-enriched uranium fuel are $5.49,11.67,16.31$, and $22.64 \mathrm{~kg}$. The calculations were done with the SAN discrete-ordinates neutron transport code and a 92 -group cross section library. The calcuiated median energy of neutrons inducing fission varies from $0.4 \mathrm{keV}$ for the one-fuelshell case to $100 \mathrm{keV}$ for the four-fuel-shell case.

\section{Data-Collection and Recording System}

The system I used is quite similar to one described elsewhere, "but I include a brief description for completeness. The system ronsi,ts of sixteen neutron detectors, circuitry and a system clock for processing and tagging the data, and an LSI-11/23 microcomputer for storing and andiyzing the data. Figure A-4 is a block diagram of the system.

$\therefore$ fraction of the neutrons that leak from the muitiplying assembly is detected by the sixteen neutron detectors, which are tubes filled with helium-3. Each is approximately 2 in. in diameter and 36 in. long; the tubes are arranged in two banks of eight, backed by polyethylene. Each tube has its own preamplifier-discriminator and amplifier mounted on the tube as'-embly. The sinteen independent detector channels provide a better signal-to-noise ratio than a large capacitance parallel combination. Another advantage: deadtime is reduced because, while one detector is recording a neutron event, the other fifteen are still active.

Data pass from the preamplifier-discriminator-amplifier module through optical coupling that ensures proper matching and reduces noise pickup. Each input line has a synchronizing circuit that allows random input signals to be synchronized with the next $1-\mu$ s pulse from the system clock. The system clock consists of a $16-\mathrm{MHz}$ crystal source a divide-by-16 precounter that delivers $1-\mathrm{MHz}$ pulses, and a 16-bit binary synchronous scaler that delivers time information. The scaler counts $65,5361-\mu \mathrm{s}$ time intervals before it restarts. Pulses occurring during each clock time are latched into a 16-bit register; each bit is assigned to a particular detector. The clock also latches a 16bit counter in a register that is used as a time tag for the data bits. Hence, an ordered stream of word pairs $\left(n_{i}, t_{i}\right)$ is generated, where $n_{i}$ is the detector identification number and $t_{i}$ is the clock time at which detector number $n_{i}$ fired. Every $65,536 \mu \mathrm{s}$, when the time counter overflows, a gate is 


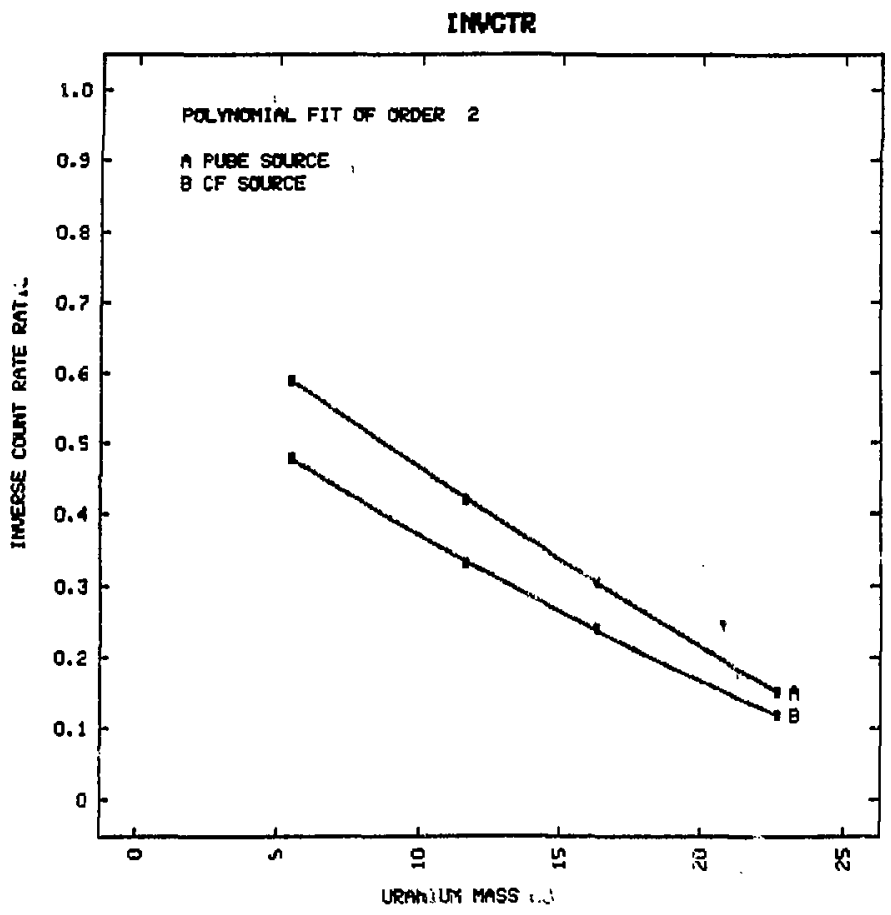

Fig. A-2. Apparent leakage multiplication of the subcritical assembly vs fuel loading. The circunstances were as follows: (1) praximuin moderator mass of $700 \mathrm{~g}$, (2) maximum reflector thickness of $10 \mathrm{~cm}$, (3) counts taken with two 3-tube banks of $\mathrm{BF}_{3}$ tubes, each tube $2.5 \mathrm{~cm}$ in diameter and $30 \mathrm{~cm}$ long, each tube bank embedded in a polyethylene block $17 \mathrm{~cm}$ in diameter and $38 \mathrm{~cm}$ long, 44) base or normalizing count taken with no fuel shells in place. Nomialal range of accumulated counts is 10,000 tc 95,000 . Note the greater apparent effectiveness of californium source neutrons.

enabled to the bus and a unique word pair is generated to identify clock cycle completion. Observe that the sequence of pairs $\left(n_{i}, t_{i}\right)$, ordered by increasing time, contains all the information that can be obtained from the detectors. There is no destruction of information by collapsing counts into time bins or by averaging operations. Thus I can process the data record with several algorithms and compare their performances.

The data and time words go to the bus sequentially and are loaded into a first-in, first-out buffer. This FIFO device, with its temporary storage capacicy of 32 events, provides a storage place for closely spaced events until the computer is ready to accspt them. The data then go to a direct memory access card (DMA) in the computer and are deposited in memory. When a block of memory is full, it is dumped to disk; this process continues until the experiment is over or the disk is full. Data can be stored permanently on floppy disks and are then available for preliminary analysis on the LSI-11/23 computer and further analysis on the CDC 7600 computer. 


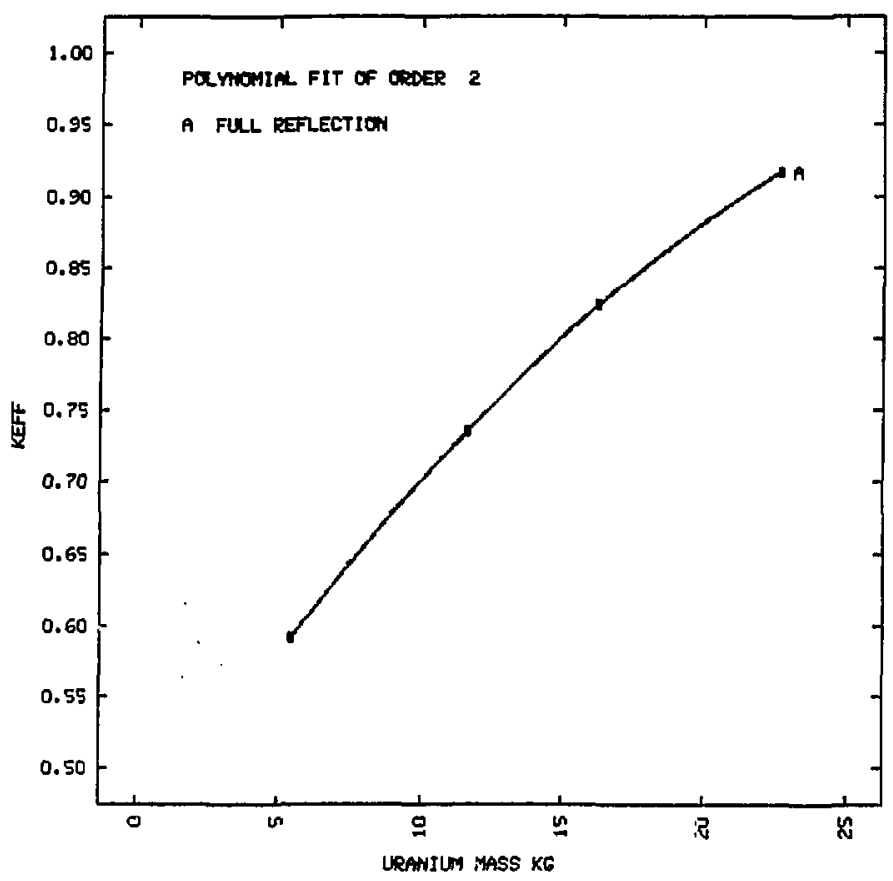

Fig. A-3. Calculated muitiplication factors of the four subcritical assemblies. Calculations were performed with the SAN discrete-ordinates transport code, using a 92-group cross section library.

\section{Collection of Data}

Figure A-5 shows a sct:ematic (approximately to scale) of the arrangement of the multiplying assembly and the detector banks that existed during the data-collection phase of the work. The circle in the center represents the outer surface of the $10-\mathrm{cm}$-thick reflector that surrounded the enriched uranium shells. The detector banks were oriented such that the helium-3 tubes were located betwr en the multiplying assembly and the 2-in.-thick polyethylene slabs that are part of the detector bax $k s$. The set of heliur 3 tubes divides into four classes when the solid angle that each subtends at the center of the multiplying assembly is calculated: $1.15 \%, 1.18 \%, 1.20 \%$, and $1.21 \%$. By solid angle I mean that subtended by a certain rectangle associated with each detector. The rectangle in question is the one generated by the intersection with the surface of the helium- 3 tube of the plane that contains the long axis of the tube and is normal to the line that contains the center of the tube and the center of the multiplying assembly. Thus reckoned, the solid angle subtended by all detectors is $19 \%$.

The vertical distance from the floor (concrete) to the center of the multiplying assembly was approximately 45 in.; the horizontal distance from the nearest wall (concreie) to the center of the assembly was approximately $63 \mathrm{in}$. Room-return effects were probably comparable to those that one would encounter in appiications. 


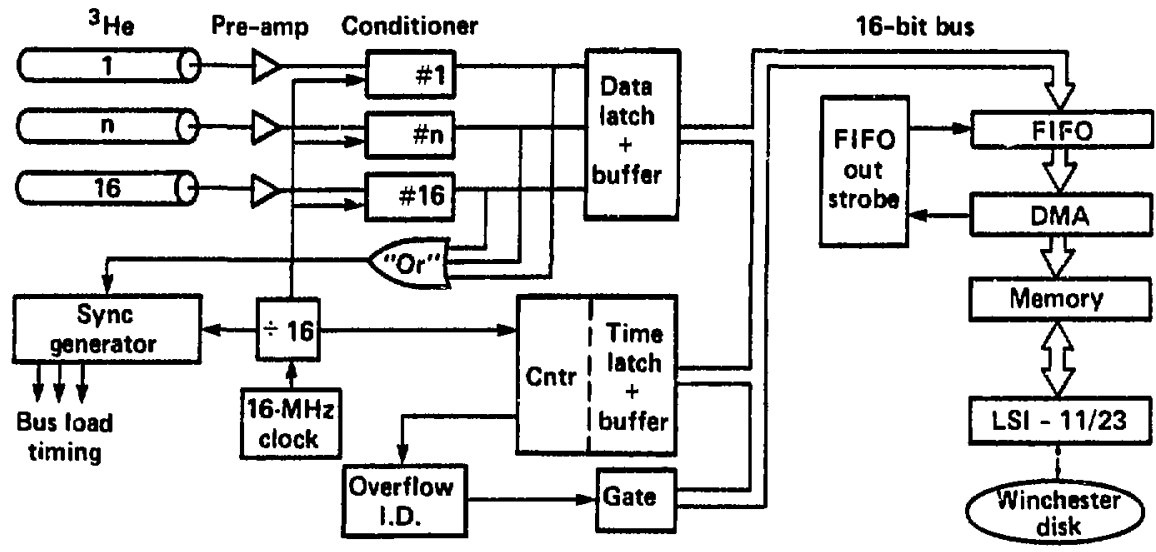

Fig. A-4. Block diagram of the data-collection and recording system. 


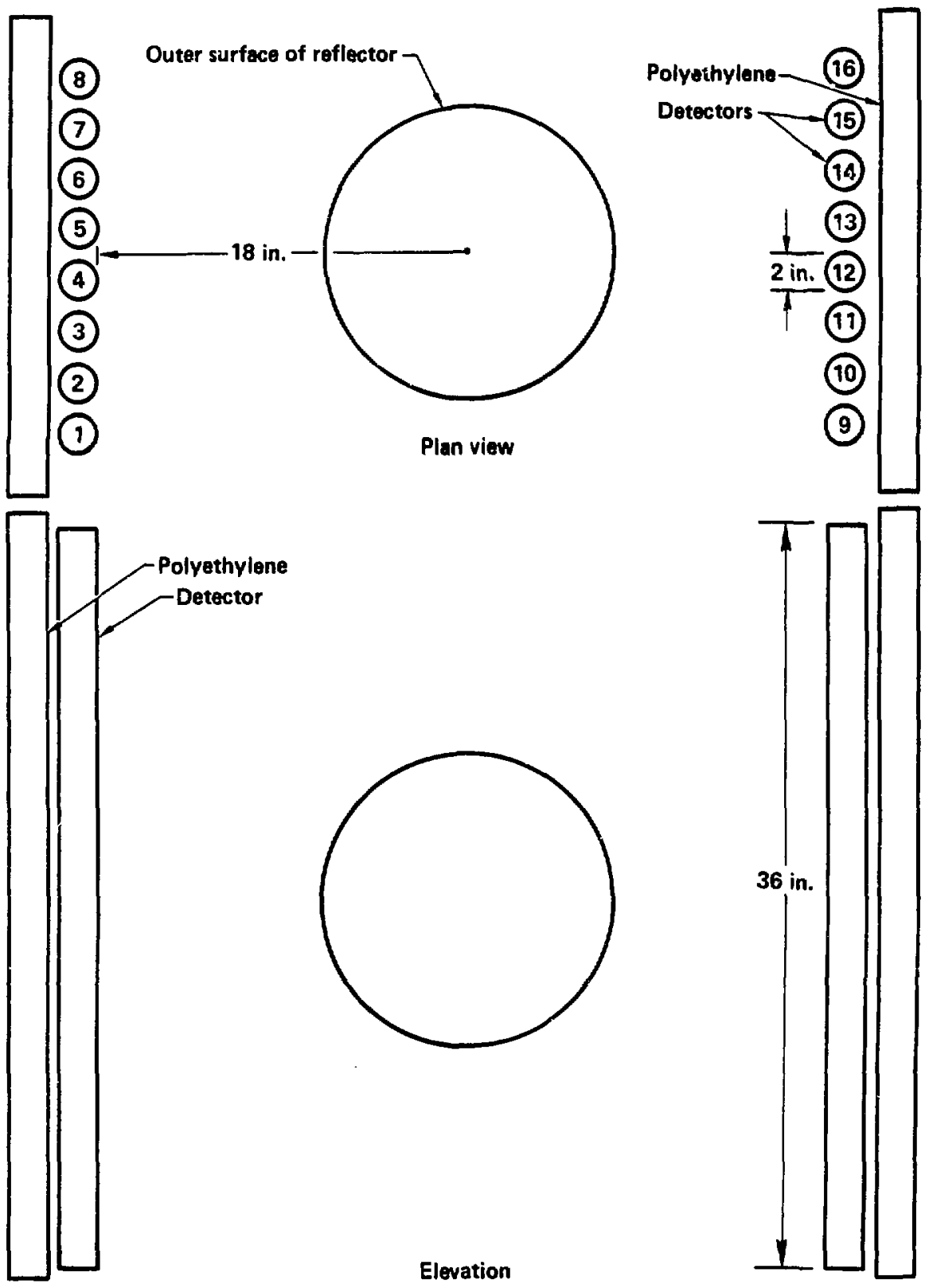

Fig. A-5. Schematic, approximately to scale, of the assembly and detector arrangement during the data-collection phase of the work. 


\section{An Experimental Study of Neutron Noise with Criticality Safety Applications in Mind}

\section{Contents}

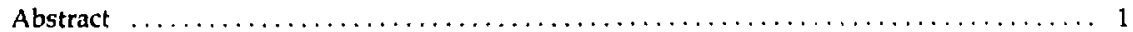

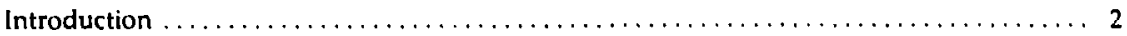

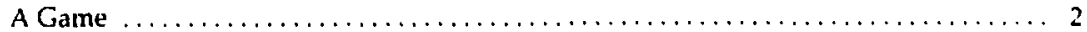

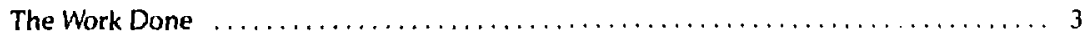

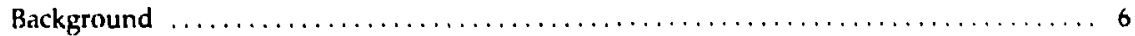

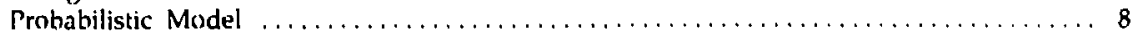

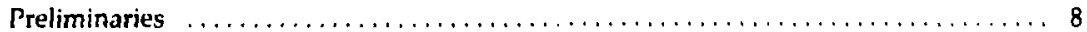

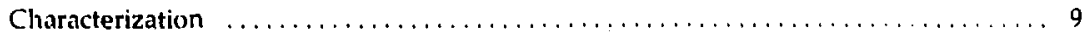

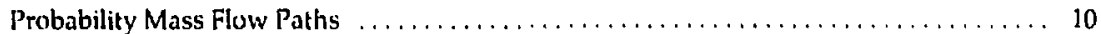

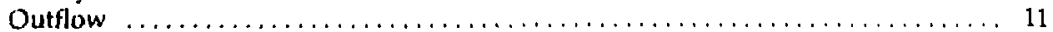

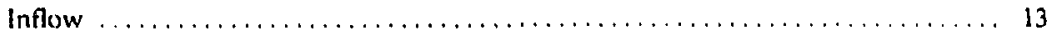

Differential Equations Satisfied by Joint Probability Mass Function $\ldots \ldots \ldots \ldots \ldots \ldots$. 15

Partial Differential Equation Satisfied by Probability Generating Function . . . . . . . . 17

Differential Equation System Satisfied by Selected Moments $\ldots \ldots \ldots \ldots \ldots \ldots \ldots \ldots 18$

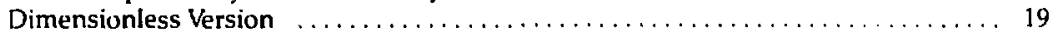

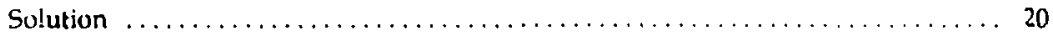

Variance-to-Mean and Covariance-to-Mean Ratios $\ldots \ldots \ldots \ldots \ldots \ldots \ldots \ldots \ldots \ldots \ldots$

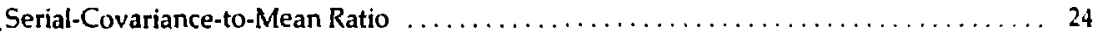

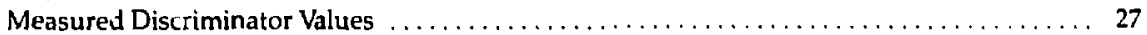

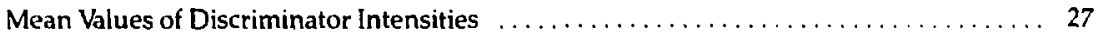

Asymptotic Increment in the Variance-to-Mean Ratio $\ldots \ldots \ldots \ldots \ldots \ldots \ldots \ldots \ldots 27$

Asymptotic Value of the Covariance-to-Mean Ratio . . . . . . . . . . . . . . . . 29

Coefficient of the Serial-Covariance-to-Mean Ratio Function $\ldots \ldots \ldots \ldots \ldots \ldots, \quad 32$

Influence of Extraneous Background on Discriminators $\ldots \ldots \ldots \ldots \ldots \ldots \ldots, 32$

Mean Values of Estimators of Assembly Decay Constant . . . . . . . . . . . . . . . . . 39

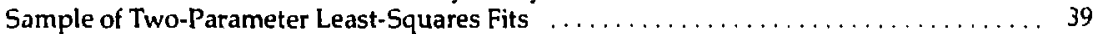

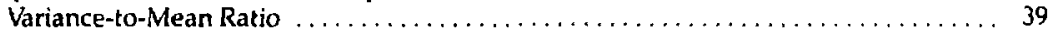

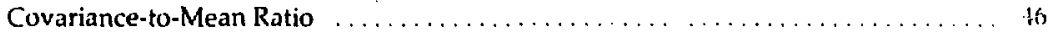

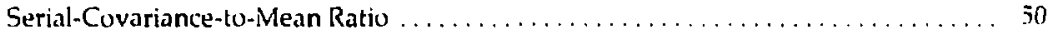

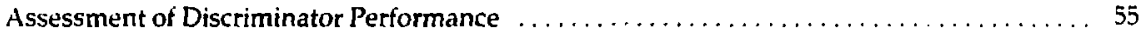

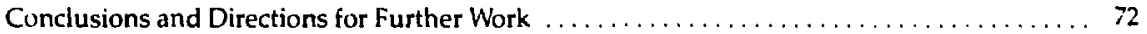

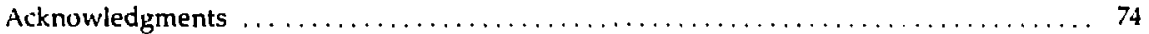

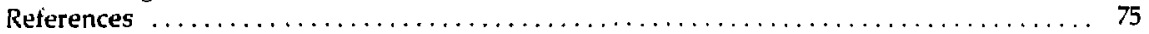

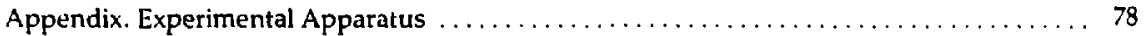

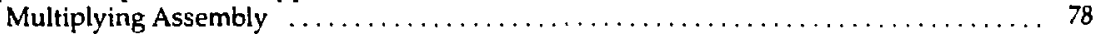

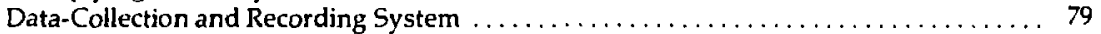

Collection of Data $\ldots \ldots \ldots \ldots \ldots \ldots \ldots \ldots \ldots \ldots \ldots \ldots \ldots \ldots \ldots \ldots \ldots \ldots$ 